Universalidade para homeomorfismos do círculo suaves por pedaços 


\title{
Universalidade para homeomorfismos do círculo suaves por pedaços
}

\author{
Kleyber Mota da Cunha
}

Orientador: Prof. Dr.Daniel Smania Brandão

Tese apresentada ao Instituto de Ciências Matemáticas e de Computação - ICMC-USP, como parte dos requisitos para obtenção do título de Doutor em Ciências - Matemática. VERSÃO REVISADA. 
Ficha catalográfica elaborada pela Biblioteca Prof. Achille Bassi e Seção Técnica de Informática, ICMC/USP, com os dados fornecidos pelo(a) autor(a)

Cu Cunha, Kleyber Mota
Universalidade para homemomorfismos do círculo
suaves por pedaços / Kleyber Mota Cunha; orientador
Daniel Smania -- São Carlos, 2011.
109 p.
Tese (Doutorado - Programa de Pós-Graduação em
Matemática)-- Instituto de Ciências Matemáticas e
de Computação, Universidade de São Paulo, 2011.
1. homeomorfismos do círculo. 2. transformação de
intercâmbio de intervalos. 3. renormalização. 4.
universalidade. 5. rigidez. I. Smania, Daniel,
orient. II. Título.


A mente que se abre a uma nova idéia jamais voltará ao seu tamanho original. (A. Einstein) 



\section{Agradecimentos}

Ao Prof. Daniel Smania, pela Matemática que me foi apresentada e pela amizade. Sua valiosa orientação foi imprescidível para a realização deste trabalho. Muito obrigado.

Aos amigos: Catiana Casonato, Fernando Micena, Flank Bezerra, Hartaman, Jean Venato, Juliano Oler, Marcela, Márcio Fenille, Marcus Bronzi, Marcos Pimenta, Mario Henrique, Thais Muniz, Thiago Catalan(Ubarana), Romero Melo, Suzete Afonso, Vando Narciso, Wescley Bonomo, por fazerem esta estada em São Carlos divertida e pela solidariedade.

Aos professores do grupo de sistemas dinâmicos do ICMC: Ali Tahzibi, Benito Pires, Carlos Gutierrez (in memorian) e Carlos Maquera, pelas aulas, conversas e ensinamentos.

A família pelo apoio de sempre e a Sandrea pela paciência e apoio.

A FAPESP pelo suporte financeiro. 

Neste trabalho nós encontramos condições suficientes para que dois homeomorfismos do círculo, $f$ e $g, C^{2+\nu}$ por pedaços serem $C^{1}$ conjugados. Além de restrições sobre a combinatória dessas aplicações (nós assumimos que elas tem algum tipo de "combinatória limitada") e uma condição necessária sobre as derivadas laterais nos pontos onde $f$ e $g$ não são diferenciáveis, nós também assumimos que a não-linearidade média de $f$ e $g$ é zero. A prova é baseada no estudo detalhado da renormalização de transformações de intercâmbio de intervalos generalizadas de genus um com certas restrições combinatoriais. 

In this work we find sufficient conditions for two piecewise $C^{2+\nu}$ homeomorphisms $f$ and $g$ of the circle to be $C^{1}$ conjugate. Besides the restrictions on the combinatorics of the maps (we assume that the maps have the same "bounded combinatorics") and necessary conditions on the one-side derivatives of points where $f$ and $g$ are not differentiable, we also assume zero mean nonlinearity for $f$ and $g$. The proof relies on the detailed study of the renormalizations of genus one generalized interval exchange maps with certain restrictions on their combinatorics. 

Resumo

Abstract

vii

Introdução

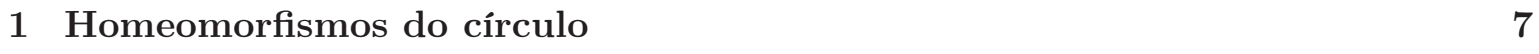

1.1 Homeomorfismos do círculo . . . . . . . . . . . . . . 7

1.1.1 Difeomorfismos do círculo . . . . . . . . . . . . . . . 8

1.1.2 Homeomorfismos suaves por pedaços . . . . . . . . . . . . . . 9

2 Transformações de Intercâmbio de Intervalos 11

2.1 Espaço das Transformações de Intercâmbio de Intervalos . . . . . . . . . . . . 12

2.2 Renormalização para Transformações de Intercâmbio de Intervalos . . . . . . 13

2.2.1 Algoritmo de Rauzy-Veech . . . . . . . . . . . . . . . . . . . . . . . . . 13

2.3 Classe de Rauzy . . . . . . . . . . . . . . . . . . . . . . . . . . . . . . . . 15

2.4 Suspensão e Genus de t.i.i.'s. . . . . . . . . . . . . . . . . . . . . 19

2.5 Cociclo de Rauzy-Veech . . . . . . . . . . . . . . . . . . . . 20

2.6 Relação entre t.i.i. de genus um e homeomorfismos do círculo . . . . . . . . . 22

2.7 Estimativas tipo Denjoy . . . . . . . . . . . . . . . . . 25

3 A Não-Linearidade e Difeomorfismos de classe $C^{2+\nu} \quad 27$

3.1 A Transformação de Möbius . . . . . . . . . . . . . . . . . . . . 29

$\begin{array}{lll}4 & \text { Teorema B } & 41\end{array}$ 
5 Teoremas C e D $\quad 45$

5.1 Representação simbólica . . . . . . . . . . . . . . . . . . . . 46

5.1.1 Caracterização dos cilindros admissíveis e suas propriedades . . . . . . 47

5.2 Prova $\operatorname{dos}$ Teoremas C e D. . . . . . . . . . . . . . . . . 53

6 Teoremas E e F $\quad 57$

6.1 Hiperbolicidade Uniforme . . . . . . . . . . . . . . . . . . . . . . . . . 58

6.1 .1 Cones Invariantes . . . . . . . . . . . . . . . . . . . . . 58

6.2 Direção Central . . . . . . . . . . . . . . . . . . . . . . . . . . . 62

6.3 Dinâmica do Cociclo de Rauzy-Veech . . . . . . . . . . . . . . . . . . . . . 68

6.4 Demonstração do Teorema E . . . . . . . . . . . . . . . . . . . . . . . 74

6.4 Métrica Projetiva . . . . . . . . . . . . . . 76

6.5 Prova do Teorema A . . . . . . . . . . . . . . . . . . . . . . 82

$\begin{array}{ll}\text { Referências Bibliográficas } & 88\end{array}$

$\begin{array}{ll}\text { Índice Remissivo } & 94\end{array}$ 


\section{Introdução}

Uma das classes mais estudadas em sistema dinâmicos é a classe dos difeomorfismos do círculo. O comportamento de tais aplicações, $f$, não é, em geral, bem entendido. Essas aplicações podem ser classificadas em termos do número de rotação que, a grosso modo, mede a taxa média de rotação das órbitas ao redor do círculo. Quando o número de rotação, $\rho(f)$, é racional, digamos $p / q, f$ deverá ter pelo menos uma órbita periódica de período $q$ (e genericamente pelo menos duas) e todas as outras órbitas deverão convergir para alguma órbita de período $q$. Por outro lado, se $\rho(f)$ for irracional, $f$ não possui órbitas periódicas e a dinâmica depende do grau de suavidade de $f$.

Nesse contexto, Poincaré provou que qualquer homeomorfismo do círculo, preservando orientação, sem órbitas periódicas é semi-conjugada a uma rotação irracional [dMvS93]. Depois, Denjoy provou que as condições: o número de rotação de $f$ é irracional, $f$ é um homeomorfismo $C^{1}$, exceto em um número finito de pontos e sua derivada $D f$ tem variação limitada, são suficientes para garantir a conjugação topológica a uma rotação. Para se obter uma maior classe de diferenciabilidade da conjugação, a teoria requer novas técnicas como por exemplo, a renormalização, e hipóteses adicionais sobre o número de rotação e a suavidade de $f$.

Em [KV91], os autores consideram uma generalização de difeomorfismos do círculo, em que a aplicação $f$ possui uma única descontinuidade $\left(x_{0}\right)$ na sua primeira derivada. Tal generalização surge naturalmente no âmbito da renormalização, em parte porque o número $\ln c=\ln \left(f^{\prime}\left(x_{0}+\right) / f^{\prime}\left(x_{0}-\right)\right)$, que é chamado de "break" de $f$ em $x_{0}$, é essencialmente invariante sob a renormalização. Quando $\ln c \neq 0$ nós dizemos que $x_{0}$ é um break-point. Eles provam que sob a renormalização qualquer aplicação com uma única descontinuidade converge para um espaço bidimensional formado por transformações de Möbius. Em [KK03a] os autores mostram que se $f$ e $g$ são dois $C^{2+\nu}$-homeomorfismos do círculo, exceto em um ponto singular $x_{0}$, com o mesmo número de rotação, tendo a expansão em frações contínuas periódicas e o mesmo valor de $c$, então $f$ e $g$ são $C^{1+\delta}$ conjugados. 
Nesse trabalho nós consideramos uma classe mais geral de homeomorfismos do círculo, que nós assumiremos ter sempre número de rotação irracional. Nós dizemos que $f: \mathbb{S}^{1} \rightarrow \mathbb{S}^{1}$ é um homeomorfismo suave por pedaços de classe $C^{r}, 0 \leq r \leq \infty$ se

- $f$ é um homeomorfismo preservando orientação;

- Existe uma partição finita de $\mathbb{S}^{1}$ por intervalos $I_{\alpha}, \alpha \in \mathcal{A}$, tal que $f$ é um difeomorfismo de classe $C^{r}$ em cada $\overline{I_{\alpha}}$.

Em vista dos resultados expostos acima, nós fazemos a seguinte pergunta:

Pergunta: Sejam $f$ e $g$ dois homeomorfismos do círculo suave por pedaços. Sob quais condições $f$ e $g$ são suavemente conjugados?

Existe uma obstrução natural para se obter conjugação suave. Se $f$ e $g$ são suavemente conjugadas então $f$ e $g$ devem possuir os mesmos breaks nos break-points correspondentes. Quando isso ocorre, nós dizemos que $f$ e $g$ são break-equivalentes. Nós dizemos que um homeomorfimo do círculo suave por pedaços não tem conexão se as órbitas dos break-points são distintas, ou seja, se $x$ e $y$ são break-points então $\left\{f^{n}(x)\right\}_{n \in \digamma} \cap\left\{f^{n}(y)\right\}_{n \in \digamma}=$, quando devem ser.

Nosso principal resultado vem ser uma resposta a essa pergunta. Mais precisamente nós provamos:

Teorema A. Sejam $f, g: \mathbb{S}^{1} \rightarrow \mathbb{S}^{1}$ homeomorfismos do círculo, $C^{2+\nu}$ por pedaços tais que

- $f$ e g não tem conexão;

- $f$ e g possuem a mesma combinatória k-limitada (ver Definição 1.1.3);

- $f$ e g são break-equivalentes;

- $\int_{0}^{1} \frac{D^{2} f(s)}{D f(s)} d s=\int_{0}^{1} \frac{D^{2} g(s)}{D g(s)} d s=0$.

Então existe uma conjugação de classe $C^{1}$ entre $f$ e g.

A principal ferramenta usada na prova do Teorema A são as transformações de intercâmbio de intervalos.

Uma transformação de intercâmbio de intervalos standard (t.i.i. standard) sobre um intervalo $I$, não degenerado e de comprimento finito, é uma aplicação $f: I \rightarrow I$ que é injetiva, sobrejetiva e localmente uma translação exceto em um número finito de descontinuidades. Assim $f$ preserva orientação e preserva a medida de Lebesgue. Exigindo que $f$ seja localmente um homeomorfismo preservando orientação, nós chamamos $f$ de uma transformação de intercâmbio de intervalos generalizada (t.i.i. generalizada). Seja $d$ o número de intervalos de continuidade de $f$. A ordem dos subintervalos antes e depois que a aplicação é iterada, constitui o que chamamos de dado combinatorial. Quando $d=2$, identificando os pontos do bordo de $I$, t.i.i.'s standard correspondem a rotações no círculo e t.i.i.'s generalizada correspondem a homeomorfismos do círculo. Nesse sentido t.i.i.'s generalizam homeomorfismos do círculo. 
Tipicamente t.i.i. standard são minimais [Kea75], mas as propriedades ergódicas das t.i.i. standard podem diferir substancialmente das rotações do círculo: elas não precisam ser ergódicas [Kea77, KN76], mas quase toda t.i.i. standard (tanto no sentido topológico [KR80], quanto no sentido de medida [Mas82, Vee82]) é ergódica.

Uma das técnicas mais utilizadas em dinâmica unidimensional é a técnica de Renormalização. Rauzy [Rau79] e Veech [Vee78] definiram um algoritmo que generaliza o clássico algoritmo das frações contínuas (que corresponde a $d=2$ ) e associa a cada t.i.i., $f$, uma outra t.i.i., $R(f)$, que é a aplicação de primeiro retorno a um subintervalo apropriado. Entretanto os dados combinatoriais de $f$ e $R(f)$ podem ser diferentes. Os possíveis dados combinatoriais para uma t.i.i. são vértices do diagrama de Rauzy; as setas desse diagrama correspondem às possíveis transições sob o algoritmo de Rauzy-Veech. A sequência de dados combinatoriais para $R^{n}(f)$ é chamada de combinatória de $f$ e pode ser visto como o "número de rotação". As componentes conexas do diagrama de Rauzy são chamadas de classes de Rauzy. Ambos os algoritmos, o de Rauzy-Veech e sua versão acelerada, que foi definida por Zorich [Zor96], são ergódicos com respeito a uma medida invariante, absolutamente contínua, no espaço das t.i.i. standard. Entretanto no algoritmo de Rauzy-Veech a medida tem massa infinita, mas a sua versão acelerada tem medida finita.

Uma t.i.i. é dita ser de classe $C^{r}$ se a t.i.i. restrita a cada elemento da partição é um difeomorfismo de classe $C^{r}$. Nós dizemos que uma t.i.i. é de genus um se $f: I \rightarrow I$ possui no máximo duas descontinuidades. Por exemplo, qualquer t.i.i. com dois ou três intervalos é de genus um. Todo homeomorfismo do círculo suave por pedaços pode ser visto como uma t.i.i. de genus um. O Teorema A segue, após algum trabalho, do seguinte teorema:

Teorema F. Sejam $f$ e $g$ duas t.i.i. de classe $C^{2+\nu}$, sem conexão, tais que

- $f$ e g possuem a mesma combinatória k-limitada;

- $f$ e g são break-equivalentes;

- As médias da não-linearidade de $f$ e g são iguais a zero, isto é,

$$
\int_{0}^{1} \frac{D^{2} f(s)}{D f(s)} d s=\int_{0}^{1} \frac{D^{2} g(s)}{D g(s)} d s=0 .
$$

Então existe $0<\lambda<1$ tal que

$$
d_{C^{2}}\left(R^{n} f, R^{n} g\right)=\mathrm{O}\left(\lambda^{\sqrt{n}}\right) .
$$

As idéias para a demonstração deste Teorema são as seguintes:

Passo 1: (Real Bounds) Nós provamos a pré-compacidade do conjunto $\left\{R^{n} f\right\}_{n \in \mathbb{N}}$ na topologia $C^{0}$ (Capítulo 4). A principal ferramenta neste passo é o Teorema de Denjoy.

Passo 2: Inspirado por [KV91], nós provamos que os pontos de acumulação da sequência $\left\{R^{n} f\right\}_{n \in \mathbb{N}}$ são t.i.i. que são Möbius em cada subintervalo da partição (Capítulo 4). Mais precisamente provamos o seguinte Teorema: 
Teorema B. Seja $f \in \mathcal{B}_{k}^{2+\nu}$. Então existe constante $C=C(k)>0$ e $0<\lambda=\lambda(k)<1$, tal que

$$
\mathrm{d}_{C^{2}}\left(\mathcal{Z}_{I_{\alpha}^{n}}\left(\hat{R}^{n}(f)\right), M_{N_{\alpha}^{n}}\right) \leq C \lambda^{n}
$$

para todo $\alpha \in \mathcal{A}$, onde

$$
N_{\alpha}^{n}=\int_{I_{\alpha}^{n}} \frac{D^{2} \hat{R}^{n}(f)(x)}{D \hat{R}^{n}(f)(x)} d x
$$

Em particular,

$$
\mathrm{d}_{C^{2}}\left(\mathcal{Z}_{I_{\alpha}^{n}}\left(\hat{R}^{n}(f)\right), \mathcal{M}\right) \leq C \lambda^{n},
$$

onde $\mathcal{M}$ é o conjunto das transformações de Möbius $M:[0,1] \rightarrow[0,1]$, tal que $M(0)=0$ e $M(1)=1$,

onde $\mathcal{B}_{k}^{2+\nu}$ é o conjunto das t.i.i.'s de classe $C^{2+\nu}$, sem conexão e com combinatória $k$-limitada.

Passo 3: Inspirados por [KS87a] e por [KK03a] nós provamos que se a média da nãolinearidade é zero então os pontos de acumulação da sequência $\left\{R^{n} f\right\}_{n \in \mathbb{N}}$ são t.i.i. afins (Capítulo 5). Precisamente provamos os seguintes Teoremas:

Teorema C. Seja $f \in \mathcal{B}_{k}^{2+\nu}$. Então existem constantes $C=C(k)>0$ e $0<\lambda_{5}=$ $\lambda_{5}(k)<1$ tal que

$$
\left|N_{\alpha}^{n}-\frac{\sum_{i=0}^{q_{\alpha}^{n}-1}\left|f^{i}\left(I_{\alpha}^{n}\right)\right|}{|I|} \int \frac{D^{2} f(x)}{D f(x)} d x\right| \leq C \lambda_{5}^{\sqrt{n}}, \text { para todo } \alpha \in \mathcal{A} .
$$

Em particula, se

$$
\int \frac{D^{2} f(x)}{D f(x)} d x=0
$$

então $\left|N_{\alpha}^{n}\right|<C \lambda_{5}^{\sqrt{n}}$ para todo $\alpha \in \mathcal{A}$.

Teorema D. Seja $f \in \mathcal{B}_{k}^{2+\nu}$ com

$$
\int \frac{D^{2} f(x)}{D f(x)} d x=0 .
$$

Então existem constantes $C=C(k)>0$ e $0<\lambda_{5}=\lambda_{5}(k)<1$ tal que

$$
\left|Z_{I_{\alpha}^{n}}\left(R^{n}(f)\right)-\mathrm{Id}\right|_{C^{2}} \leq C \cdot \lambda_{5}^{\sqrt{n}} \text { para todo } \alpha \in \mathcal{A} .
$$

Passo 4: (Linearização) Nós encontramos uma t.i.i. afim $f_{A}$ com a mesma combinatória de $f$ tal que $d_{C^{2}}\left(R^{n} f, R^{n} f_{A}\right)$ vai pra zero rapidamente (Capítulo 6). De fato, provamos o seguinte Teorema:

Teorema E. Seja $\left(f, \mathcal{A},\left\{I_{\alpha}\right\}_{\alpha \in \mathcal{A}}\right) \in \mathcal{B}_{k}^{2+\nu}$ e suponha que

$$
\int \frac{D^{2} f(x)}{D f(x)} d x=0 .
$$

Então existe uma t.i.i. $\left(f_{A}, \mathcal{A},\left\{\tilde{I}_{\alpha}\right\}_{\alpha \in \mathcal{A}}\right)$ e $0<\lambda<1$ tal que 
(i) $\left.f_{A}\right|_{\tilde{I}_{\alpha}}$ é afim para cada $\alpha \in \mathcal{A}$ e tem a mesma combinatória de $f$;

(ii) $f_{A}$ é universal, isto é, se $f$ e g satisfazem as hipóteses do Teorema, tem a mesma combinatória e são break-equivalentes (Definição 1.1.3) então $f_{A}=g_{A}$.

(iii) $d_{C^{2}}\left(R^{n} f, R^{n} f_{A}\right)=\mathrm{O}\left(\lambda^{\sqrt{n}}\right)$.

Passo 5: (Universalidade) Nós provamos que $f_{A}$ só depende da combinatória de $f$ e dos valores dos breaks nos break-points de $f$ (Capítulo 6). Mais precisamente, obtemos o seguinte Teorema:

Teorema F. Sejam $f, g \in \mathcal{B}_{k}^{2+\nu}$ tais que

- $f$ e g possuem a mesma combinatória;

- $f$ e g são break-equivalentes;

- $\int_{0}^{1} \frac{D^{2} f(s)}{D f(s)} d s=\int_{0}^{1} \frac{D^{2} g(s)}{D g(s)} d s=0$.

Então existe $0<\lambda<1$ tal que

$$
d_{C^{2}}\left(R^{n} f, R^{n} g\right)=\mathrm{O}\left(\lambda^{\sqrt{n}}\right) .
$$

Nos capítulos 1 e 2 nós estabelecemos algumas definições e propriedades sobre homeomorfismos do círculo e transformações de intercâmbio de intervalos, respectivamente, de modo a tornar essa tese auto-contida. No capítulo 3, estabelecemos algumas relações entre difeomorfismos de classe $C^{2+\nu}$ e as transformações de Möbius. 



$\left(\frac{10}{10}\right.$

\section{Homeomorfismos do círculo}

Para dar uma motivação para os nossos resultados e estabelecer algumas notações, neste capítulo, nós apresentaremos alguns resultados sobre homeomorfismos e difeomorfismos do círculo. Para mais detalhes sobre os resultados aqui apresentados, o leitor pode consultar [dMvS93] e [Her79].

\subsection{Homeomorfismos do círculo}

Considere o círculo $\mathbb{S}^{1}$ como o espaço quociente da reta real pelo grupo das translações por inteiros: $\mathbb{S}^{1}=\mathbb{R} \backslash \mathbb{Z}$. Seja $\pi: \mathbb{R} \rightarrow \mathbb{S}^{1}$ a aplicação quociente, ou seja, $\pi(x)=x+\mathbb{Z}$. Nós dizemos que $F: \mathbb{R} \rightarrow \mathbb{R}$ é um levantamento de um homeomorfismo $f: \mathbb{S}^{1} \rightarrow \mathbb{S}^{1}$ se $F$ é contínua e a igualdade

$$
f \circ \pi=\pi \circ F,
$$

é satisfeita para todo $x \in \mathbb{R}$. Seja $F_{1}, F_{2}$ dois levantamentos de $f$. Então $F_{1}-F_{2} \in \mathbb{Z}$, isto é, exite $r \in \mathbb{Z}$ tal que $F_{1}(x)-F_{2}(x)=r$ para todo $x \in \mathbb{R}$.

Suponhamos agora que $f: \mathbb{S}^{1} \rightarrow \mathbb{S}^{1}$ é um homeomorfismo preservando orientação. Então o número

$$
\tau(F):=\lim _{|n| \rightarrow \infty} \frac{1}{n}\left(F^{n}(x)-x\right)
$$

existe para todo $x \in \mathbb{R} . \tau(F)$ independe do ponto $x$ escolhido, e se $F_{1}, F_{2}$ são dois levantamentos de $f$, então $\tau\left(F_{1}\right)-\tau\left(F_{2}\right)=F_{1}-F_{2} \in \mathbb{Z}$. 
O número $\tau(f)=\pi(\tau(F))$ é chamado de número de rotação de $f$. É sabido também que a aplicação $\tau(\cdot)$ é contínua na topologia $C^{0}$ [dMvS93], e é invariante por conjugação, ou seja, se existe um homeomorfismo preservando orientação $h: \mathbb{S}^{1} \rightarrow \mathbb{S}^{1}$, então

$$
\tau\left(h \circ f \circ h^{-1}\right)=\tau(f) .
$$

É sabido que se $\tau(f)=p / q$ com $p, q$ primos entre si se, e somente se, $f$ tem pelo menos uma órbita periódica de período $q$ e todas as outras órbitas periódicas, caso existam, tem o mesmo período. Poincaré, mostrou que todo homeomorfismo preservando orientação $f: \mathbb{S}^{1} \rightarrow \mathbb{S}^{1}$, com $\tau(f) \in \mathbb{R} \backslash \mathbb{Q}$ é topologicamente semi-conjugado à rotação irracional correspondente, $R_{\tau(f)}=x+\tau(f) \bmod 1$. Seja $h$ essa semi-conjugação. Se $h$ não é uma conjugação topológica, então existe um intervalo $J \subset \mathbb{S}^{1}$, tal que $h(J)$ é um ponto. Esse intervalo $J$ é chamado intervalo errante de $f$. Mais precisamente, um intervalo $J \subset \mathbb{S}^{1}$ é um intervalo errante de $f$, se $J, f(J), \ldots, f^{n}(J), \ldots$ são intervalos dois a dois disjuntos e $f^{n}(J)$ não converge a uma órbita periódica. Assim saber se $f$ é conjugado topologicamente a $R_{\tau(f)}$ é equivalente a saber se $f$ possui intervalos errantes.

Dada uma função $\phi: \mathbb{S}^{1} \rightarrow \mathbb{R}$ nós definimos a variação de $\phi, \operatorname{Var}(\phi)$ como sendo

$$
\operatorname{Var}(\phi)=\sup \sum_{i=1}^{n}\left|f\left(x_{i+1}-f\left(x_{i}\right)\right)\right|,
$$

onde o sup acima é tomado sobre todas as partições $0=x_{1}<\ldots<x_{n}=1$. Se $\operatorname{Var}(\phi)<\infty$, nós dizemos que $\phi$ tem variação limitada. O leitor pode notar que se $\phi$ é Lipschitz então $\phi$ tem variação limitada. Usando o conceito de variação, Denjoy mostrou uma condição necessária para se obter uma conjugação entre $f$ e $R_{\tau(f)}$.

Teorema de Denjoy. Seja $f: \mathbb{S}^{1} \rightarrow \mathbb{S}^{1}$ um difeomorfismo preservando orientação de classe $C^{1}$, tal que $\tau(f) \in \mathbb{R} \backslash \mathbb{Q}$. Se $D f$ tem variação limitada então $f$ é topologicamente conjugado a $R_{\tau(f)}$

Do ponto de vista érgódico, temos que se $\tau(f) \in \mathbb{R} \backslash \mathbb{Q}$ então $f$ é unicamente ergódica e metricamente isomorfo a rotação irracional correspondente, $R_{\tau(f)}=x+\tau(f) \bmod 1$.

\subsubsection{Difeomorfismos do círculo}

Durante toda esta seção os difeomorfismos do círculo aqui considerados sempre preservarão orientação.

Uma pergunta bastante interessante no contexto de difemorfismos do círculo é quando nós podemos obter uma conjugação suave entre um difeomorfismo $f$ e sua rotação correspondente. Conjugação suave nos garante a existência de medida invariante absolutamente contínua e com densidade contínua. Outra propriedade interessante é que se $f_{1}$ e $f_{2}$ são dois homeomorfismos do círculo $C^{1}$ conjugados, então as propriedades métricas de $f_{1}$ e $f_{2}$ coincidem assintoticamente.

Resultados de suavidade para conjugações foram primeiro obtidos por Arnol'd [Arn61]. Ele provou que se $f$ é um difeomorfismo, perto do conjunto das rotações, analítico e $\tau(f)$ é um 
número Diofantino de classe $D_{1}$ então a conjugação entre $f$ e $R_{\tau(f)}$ é analítica. Lembramos que um núemro $\tau$ pertence a classe $D_{\delta}, \delta>0$, se existe uma constante $C=C(\tau)>0$ tal que

$$
\left|\tau-\frac{p}{q}\right| \geq \frac{C}{q^{2+\delta}}
$$

para qualquer inteiro $p$ e qualquer inteiro positivo $q$. Ou seja. o número $\tau$ é mal aproximado por racionais. Para qualquer $\delta>0$ o complemento do conjunto $D_{\delta}$ tem medida de Lebesgue zero. Arnol'd também construiu um exemplo mostrando que a condição genérica $\tau \in D_{\delta}$ é essencial e que a teoria de Denjoy não pode ser estendida para todos os número irracionais. Mais precisamente, Arnol'd mostrou que no caso em que $\tau(f)$ é bem aproximado por racionais, uma medida invariante de um difeomorfismo analítico pode ser essencialmente singular com respeito à medida de Lebesque, o que neste caso, significa que a conjugação não é suave. Arnol'd também conjecturou que a condição local de que $f$ seja próxima ao conjunto das rotações não é essencial, e que um resultado similar deveria valer sem esta condição.

Um resultado resultado deste tipo foi primeiro obtido por Herman. Ele provou ([Her79], [Her80]) que se $f$ é $C^{k}, k \geq 3$ e $\tau \in \cap D_{\delta}$ a conjugação de $f$ com sua rotação $R_{\tau(f)}$ é suave. A teoria de Herman foi depois estendida por Yoccoz [Yoc84] para uma classe mais ampla de números de rotação. Depois Khanin e Sinai ([SK89],[KS87a]) e também Katznelson e Ornstein ([KO89a],[KO89b]) mostraram que resultados deste tipo, podem ser construídos para difeomorfismos de classe $C^{2+\nu}$.

\subsubsection{Homeomorfismos suaves por pedaços}

Rotações lineares desempenham um papel de destaque na teoria de rigidez dos difeomorfismos suaves. Mas encontrar conjugação suave com uma rotação linear é impossível no caso de difeomorfismos com singularidades. Aqui o termo "difeomorfismo com singularidades" é usado para enfatizar que as aplicações são suaves e inversíveis em quase todo $\mathbb{S}^{1}$, exceto nos pontos singulares. Então o principal problema agora é saber se existe uma conjugação suave entre dois difeomorfismos $f_{1}$ e $f_{2}$ com o mesmo "tipo" de pontos singulares. Claro que também é necessário assumir que os números de rotação dos difeomorfismos são irracionais e iguais.

Definição 1.1.1. Nós dizemos que $f: \mathbb{S}^{1} \rightarrow \mathbb{S}^{1}$ é um homeomorfismo de classe $C^{r}, r \geq 1$, por pedaços, se

(i) $f$ é um homeomorfismo preservando orientação;

(ii) Existe uma partição finita de $\mathbb{S}^{1}$ por intervalos $I_{\alpha}, \alpha \in \mathcal{A}$, tal que $f$ é um difeomorfismo de classe $C^{r}$ em cada $\overline{I_{\alpha}}$.

O conjunto das aplicações $f: \mathbb{S}^{1} \rightarrow \mathbb{S}^{1}$ satisfazendo a Definição 1.1.1 e com número de rotação irracional será denotado por $\mathcal{B}^{r}$.

Neste trabalho, nós damos uma resposta para a seguinte questão:

Questão: Sobre que condições $f, g \in \mathcal{B}^{2+\nu}$ são $C^{1}$ conjugadas? 
Existe uma obstrução natural para se obter uma conjugação suave $h$ entre duas transformações de intercâmbio de intervalos generalizadas $f$ e $g$.

Definição 1.1.2 (Break; Break Point). Seja $f \in \mathcal{B}^{1}$ O número real

$$
B P_{f}(x):=\ln \frac{D_{-} f(x)}{D_{+} f(x)}
$$

é chamado break de $f$ em $x$, onde $D_{-} f(x)$ e $D_{+} f(x)$ são as derivadas laterais à esquerda e à direita de $x$, respectivamente. Se $B P_{f}(x) \neq 0$ dizemos que $x$ é um break point. Denotaremos por $B P_{f}$ o conjunto dos break points de $f$.

Seja $f, g \in \mathcal{B}^{1}$ e suponhamos que $f$ e $g$ são $C^{1}$-conjugadas, ou seja, existe $h: \mathbb{S}^{1} \rightarrow \mathbb{S}^{1}$ de classe $C^{1}$ tal que $h \circ f=g \circ h$. Então

$$
h\left(B P_{f}\right)=B P_{g} \text { e } B P_{f}(x)=B P_{g}(h(x)) .
$$

Definição 1.1.3. Sejam $f, g \in \mathcal{B}^{1} e 0<x_{1}<\ldots<x_{d-1}<1$ e $0<y_{1}<\ldots<y_{d-1}<1$ os breaks points de $f$ e $g$ respectivamente. Nós dizemos que $f$ e $g$ são break-equivalentes se

$$
B P_{f}\left(x_{i}\right)=B P_{g}\left(y_{i}\right), \text { para todo } i=1, \ldots, d-1 .
$$

Aplicações no conjunto $\mathcal{B}^{2+\nu}$ com um único break point foram primeiro consideradas por Vul e Khanin ([KV91]). Os autores mostram que a as renormalizações dessas aplicações convergem, na topologia $C^{2}$, para o espaço bidimensional formado por transformações de Möbius.

Ainda no contexto de um único break point, temos o seguinte resultado [KK03a]: Seja $f, g \in \mathcal{B}^{2+\nu} \operatorname{com} \tau(f)=\tau(g)=a+\sqrt{b} ; a, b \in \mathbb{Q}$, e $x_{f}$ e $x_{g}$ os break points de $f$ e $g$, respectivamente, com $B P_{f}\left(x_{f}\right)=B P_{g}\left(x_{g}\right) \neq 1$. Então existe $\gamma>0$, tal que $f$ e $g$ são $C^{1+\gamma}$ conjugadas. 


$\frac{10}{2}$

\section{Transformações de Intercâmbio de}

Intervalos

Neste capítulo, definimos o nosso objeto de estudo, que são as transformações de intercâmbio de intervalos, estabelecemos algumas notações a serem usadas no restante do texto, bem como apresentaremos alguns resultados conhecidos sobre as mesmas. Por este motivo não demonstraremos a maioria deles, mas deixaremos indicado onde o leitor poderá encontrar suas respectivas demonstrações.

Seja $I$ um intervalo ${ }^{1}, \mathcal{A}$ um conjunto finito, que chamaremos de alfabeto com $d \geq 2$ elementos e $\mathcal{P}=\left\{I_{\alpha}: \alpha \in \mathcal{A}\right\}$ uma partição indexada por $\mathcal{A}$ de subintervalos de $I$. Nós dizemos que a tripla $(f, \mathcal{A}, \mathcal{P})$, onde $f: I \rightarrow I$ é uma bijeção, é uma transformação de intercâmbio de intervalos generalizada (t.i.i. generalizada), se $\left.f\right|_{I_{\alpha}}$ é um homeomorfismo preservando orientação para cada $\alpha \in \mathcal{A}$.

Quando $\left.f\right|_{I_{\alpha}}$ é uma translação, nós dizemos que $f$ é uma transformação de intercâmbio de intervalos standard (t.i.i. standard). T.i.i.'s standard preservam a medida de Lebesgue e aparecem naturalmente como aplicações de primeiro retorno de fluxos geodésicos numa superfície de translação [Zor96]. Mas elas também são um bom exemplo de um sistema dinâmico simples que tem uma dinâmica muito rica.

\footnotetext{
${ }^{1}$ Todos os intervalos deverão ser limitados, fechados à esquerda e abertos à direita. Por simplicidade, nós assumiremos que o ponto inicial de $I$ é 0 .
} 
No caso em que não precisamos fazer distinção, ou enfatizar, se $f$ é uma t.i.i. standard ou uma t.i.i. generalizada, chamaremos $f$ de t.i.i. por simplicidade.

Quando $d=2$ identificando os pontos iniciais e finais de $I$, temos que t.i.i. standard corresponde a rotações do círculo e t.i.i. generalizadas corresponde a homeomorfismos do círculo.

A ordem dos subintervalos da partição $I_{\alpha}$ antes e depois da iteração de $f$ constituem o dado combinatorial de $f$, que denotaremos pelo par $\pi=\left(\pi_{0}, \pi_{1}\right)$, onde $\pi_{\varepsilon}: \mathcal{A} \rightarrow\{1, \ldots, d\}$ são bijeções para todo $\varepsilon \in\{0,1\}$.

Uma t.i.i. standard é unicamente determinada pelo par $(\pi, \lambda)$, onde $\lambda=\left(\lambda_{\alpha}\right) \in \mathbb{R}_{+}^{\mathcal{A}}$ é tal que $\left|I_{\alpha}\right|=\lambda_{\alpha}$, onde $\left|I_{\alpha}\right|$ denota o comprimento do intervalo $I_{\alpha}$. O vetor $\lambda$ é chamado vetor partição. Nós chamamos

$$
p=\pi_{1} \circ \pi_{0}^{-1}:\{1, \ldots, d\} \rightarrow\{1, \ldots, d\},
$$

de monodromia invariante do par $\pi=\left(\pi_{0}, \pi_{1}\right)$.

Nós dizemos que o par $\pi=\left(\pi_{0}, \pi_{1}\right)$ é redutivel se existe $k \in\{1, \ldots, d-1\}$ tal que

$$
\pi_{1} \circ \pi_{0}^{-1}(\{1, \ldots, k\})=\{1, \ldots, k\},
$$

caso contrário, nós dizemos que $\pi=\left(\pi_{0}, \pi_{1}\right)$ é irredutivel. Se $\pi$ é redutível, então para qualquer escolha de $\lambda \in \mathbb{R}_{+}^{\mathcal{A}}$, o subintervalo

$$
J=\bigcup_{\pi_{0}(\alpha) \leq k} I_{\alpha}=\bigcup_{\pi_{1}(\alpha) \leq k} I_{\alpha}
$$

e o seu complementar, são invariantes pela t.i.i. standard definida por $f=(\pi, \lambda)$. Isto significa que $f$ se decompõe em duas novas t.i.i. standard com dado combinatorial mais simples. Nós denotaremos por $\Pi_{d}$, o conjunto das permutações de $d$ elementos, irredutíveis.

\subsection{Espaço das Transformações de Intercâmbio de Intervalos}

Considere $J$ um intervalo e $f: J \rightarrow f(J)$ uma aplicação. Seja $[a, b] \subset J$ um intervalo tal que $f$ seja um homemorfismo preservando orientação. Nós definimos o Zoom de $f$ em $[a, b]$ e denotamos por $\mathrm{Z}_{[a, b]} f$, a aplicação $\mathrm{Z}_{[a, b]} f:[0,1] \rightarrow[0,1]$ definida por $\mathrm{Z}_{[a, b]} f=A_{2} \circ f \circ A_{1}$, onde $A_{1}$ é a aplicação afim, preservando orientação, que leva $[0,1]$ em $[a, b]$ e $A_{2}$ é a aplicação afim, preservando orientação, que leva $[f(a), f(b)]$ em $[0,1]$.

Assim para cada transformação de intercâmbio de intervalos generalizada, nós podemos associar a seguinte quádrupla:

$$
\left(\pi,\left(\left|I_{\alpha}\right|\right)_{\alpha \in \mathcal{A}},\left(\left|f\left(I_{\alpha}\right)\right|\right)_{\alpha \in \mathcal{A}},\left(Z_{I_{\alpha}} f\right)_{\alpha \in \mathcal{A}}\right) \in \Pi_{d} \times \Delta_{d-1} \times \Delta_{d-1} \times \operatorname{Hom}_{+}([0,1],[0,1]),
$$

onde $\operatorname{Hom}_{+}([0,1],[0,1])$ é o conjunto dos homeomorfismos $h:[0,1] \rightarrow[0,1]$, preservando orientação, tal que $h(0)=0$ e $h(1)=1$. 
Se trocarmos o conjunto $\operatorname{Hom}_{+}([0,1],[0,1])$ pelo conjunto $\operatorname{Diff}_{+}^{r}([0,1],[0,1])$ dos difeomorfismos de classe $C^{r}, 0 \leq r \leq \infty$ preservando orientação que fixam os pontos 0 e 1 , nós obtemos o espaço das trasnformações de intercâmbio de intervalos de classe $C^{r}$. Se trocarmos $\operatorname{Hom}_{+}([0,1],[0,1])$ pelo conjunto $\{\mathrm{Id}\}$ teremos o espaço das transformações de intercâmbio de intervalos afins. Se além dito, trocarmos $\Delta_{d-1} \times \Delta_{d-1}$ pelo conjunto $\left\{(\lambda, \lambda), \lambda \in \Delta_{d-1}\right\}$ obtemos o espaço das transformações de intercâmbio de intervalos standard.

No conjunto das trasformações de intercâmbio de intervalos generalizadas de classe $C^{r}$, nós definimos a distância, na topologia $C^{r}$ por

$$
\begin{aligned}
d_{C^{r}}(f, g):= & \max _{\alpha \in \mathcal{A}}\left\{\left\|Z_{I_{\alpha}} f-Z_{\tilde{I}_{\alpha}} g\right\|_{C^{r}}\right\}+ \\
& \left\|\left(I_{\alpha}\right)_{\alpha \in \mathcal{A}}-\left(\tilde{I_{\alpha}}\right)_{\alpha \in \mathcal{A}}\right\|_{1}+\left\|\left(f\left(I_{\alpha}\right)\right)_{\alpha \in \mathcal{A}}-\left(g\left(\tilde{I}_{\alpha}\right)\right)_{\alpha \in \mathcal{A}}\right\|_{1},
\end{aligned}
$$

onde $\|\cdot\|_{C^{r}}$ denota a norma do sup na topologia $C^{r}$ e $\|\cdot\|_{1}$ denota a norma da soma.

\subsection{Renormalização para Transformações de Intercâmbio de Intervalos}

Uma das técnicas mais utilizadas em dinâmica unidimensional é a renormalização, que significa escolher um intervalo $J \subset[0,1)$ e calcular a transformação induzida $f_{J}: J \rightarrow J$ dada por $f_{J}(x)=f^{n(x)}(x)$, onde $n(x)$ é o tempo de primeiro retorno do ponto $x$ ao intervalo $J$. Evidentemente, nem sempre a transformação induzida está bem definida.

\subsubsection{Algoritmo de Rauzy-Veech}

Seguindo o algoritmo de Rauzy [Rau79] e Veech [Vee78] para cada t.i.i. f, nós consideraremos, caso existam, aplicações de primeiro retorno $f_{n}$ de $f$ em uma sequência de intervalos decrescentes $I^{n}$ tendo o mesmo ponto final à esquerda de $I$. Essas aplicações $f_{n}$ são ainda uma t.i.i. com o mesmo alfabeto $\mathcal{A}$, mas o dado combinatorial de $f_{n}$ pode ser diferente do dado combinatorial de $f$.

Dado dois intervalos $J$ e $U$, nós escrevemos $J<U$ se $\operatorname{int}(J) \cap \operatorname{int}(U)=\varnothing$ e $x<y$ para todo $x \in J$ e para todo $y \in U$, onde $\operatorname{int}(A)$ denota o interior do conjunto $A$. Isto define uma ordem parcial, no conjunto de todos os intervalos. Pela definição de $\pi=\left(\pi_{0}, \pi_{1}\right)$ temos que

$$
I_{\alpha}<I_{\beta}
$$

se e somente se $\pi_{0}(\alpha)<\pi_{0}(\beta)$ e

$$
f\left(I_{\alpha}\right)<f\left(I_{\beta}\right)
$$

se e somente se $\pi_{1}(\alpha)<\pi_{1}(\beta)$.

Para cada $\varepsilon \in\{0,1\}$, defina $\alpha(\varepsilon)=\pi_{\varepsilon}^{-1}(d)$. Se $\left|I_{\alpha(0)}\right| \neq\left|f\left(I_{\alpha(1)}\right)\right|$ nós dizemos que $f$ é Rauzy-Veech-renormalizável, ou simplesmente renormalizável, de agora em diante. Se $\left|I_{\alpha(0)}\right|>\left|f\left(I_{\alpha(1)}\right)\right|$ dizemos que a letra $\alpha(0)$ é vencedora, a letra $\alpha(1)$ é a perdedora e que $f$ 
tem tipo 0. Então nós podemos definir uma aplicação $\hat{R}(f)$, que é a aplicação de primeiro retorno de $f$ ao intervalo

$$
I^{1}=I \backslash f\left(I_{\alpha(1)}\right) .
$$

Se $\left|I_{\alpha(0)}\right|<\left|f\left(I_{\alpha(1)}\right)\right|$, a letra $\alpha(1)$ é a vencedora, a letra $\alpha(0)$ é a perdedora e dizemos que $f$ tem tipo 1. Então, neste caso, podemos definir também $\hat{R}(f)$, como sendo a aplicacão de primeiro retorno de $f$ ao intervalo

$$
I^{1}=I \backslash I_{\alpha(0)}
$$

Podemos ver $\hat{R}(f)$ como uma t.i.i., cuja partição do domínio $\mathcal{P}^{1}$ é também indexada por $\mathcal{A}$, do seguinte modo: denote por $I_{\alpha}^{1}$ os subintervalos de $I^{1}$, e defina-os como sendo

$$
I_{\alpha}^{1}= \begin{cases}I_{\alpha}, & \text { se } \alpha \neq \alpha(0) \\ I_{\alpha(0)} \backslash f\left(I_{\alpha(1)}\right), & \text { se } \alpha=\alpha(0),\end{cases}
$$

se $f$ tem tipo 0 , e

$$
I_{\alpha}^{1}= \begin{cases}I_{\alpha}, & \text { se } \alpha \neq \alpha(1), \alpha(0) \\ f^{-1}\left(f\left(I_{\alpha(1)}\right) \backslash I_{\alpha(0)}\right), & \text { se } \alpha=\alpha(1) \\ f^{-1}\left(I_{\alpha(0)}\right), & \text { se } \alpha=\alpha(0),\end{cases}
$$

se $f$ tem tipo 1. É fácil ver que em ambos os casos (tipos 0 e 1) nós temos

$$
\hat{R}(f)(x)= \begin{cases}f^{2}(x), & \text { se } x \in I_{\alpha(1-\varepsilon)}^{1}, \\ f(x), & \text { caso contrário, }\end{cases}
$$

e $\left(\hat{R}(f), \mathcal{A}, \mathcal{P}^{1}\right)$ é uma t.i.i. chamada indução de Rauzy-Veech, ou simplesmente, renormalização, de $f$. Se $f$ é renormalizável com tipo $\varepsilon \in\{0,1\}$ então o dado combinatorial $\pi^{1}=\left(\pi_{0}^{1}, \pi_{1}^{1}\right)$ de $\hat{R}(f)$ é dado por

$$
\pi_{\varepsilon}^{1}:=\pi_{\varepsilon}
$$

e

$$
\pi_{1-\varepsilon}^{1}(\alpha)= \begin{cases}\pi_{1-\varepsilon}(\alpha), & \text { se } \pi_{1-\varepsilon}(\alpha) \leq \pi_{1-\varepsilon}(\alpha(\varepsilon)), \\ \pi_{1-\varepsilon}(\alpha)+1, & \text { se } \pi_{1-\varepsilon}(\alpha(\varepsilon))<\pi_{1-\varepsilon}(\alpha)<d, \\ \pi_{1-\varepsilon}(\alpha(\varepsilon))+1, & \text { se } \pi_{1-\varepsilon}(\alpha)=d .\end{cases}
$$

Como $\pi^{1}$ depende somente de $\pi$ e do tipo $\varepsilon$, nós usaremos a notação $r_{\varepsilon}(\pi)=\pi^{1}$.

Considerando $I=[0,1)$ e reescalonando o domínio de $\hat{R}(f)$ para o intervalo $[0,1)$ nós temos definida a aplicação $R f:[0,1) \rightarrow[0,1)$ que é uma t.i.i. com o mesmo número de intervalos de $f$ e denominada renormalização de $f$. Quando $R^{n} f$ está definida para todo $n \geq 0$ nós dizemos que $f$ é infinitamente renormalizável. Nosso próximo passo é estabelecer uma condição necessária e suficiente para tal. 
Seja $\partial I_{\alpha}$ o ponto final a esquerda de cada subintervalo $I_{\alpha}$. Relembrando que nós assumimos o ponto inicial de $I$ como sendo 0. Então

$$
\partial I_{\alpha}=\sum_{\pi_{0}(\eta)<\pi_{0}(\alpha)} \lambda_{\eta}
$$

representa a extremidade esquerda de cada subintervalo $I_{\alpha}$.

Definição 2.2.1 (Conexão). Dizemos que uma tripla $(\alpha, \beta, m) \in \mathcal{A} \times \mathcal{A} \times \mathbb{N}$ é uma conexão para $f$ se

$$
f^{m}\left(\partial I_{\alpha}\right)=\partial I_{\beta} \quad \text { para algum } m \geq 1 \text { e } \alpha, \beta \in \mathcal{A}, \operatorname{com} \pi_{0}(\beta) \neq 1
$$

É claro que se $f$ não tem conexão então $\hat{R}(f)$ está bem definida e não possui conexão. Assim a condição de $f$ não ter conexão é suficiente para que todos os iterados $\hat{R}^{n}(f), n \geq 0$ estejam definidos. De fato Keane [Kea75] mostrou que a condição de não ter conexão é necessária e sificiente para que $\hat{R}(f)$ esteja definido para todo $n \geq 0$. Ele também mostrou que se uma t.i.i. standard não tem conexão então ela é minimal.

Observação 2.2.1. Quando $d=2$, a condição de $f$ não ter conexão é equivalente ao número de rotação de $f$ ser irracional.

Dada uma t.i.i. $f$ sem conexão, a sequência $\left\{\pi^{i}, \varepsilon^{i}\right\}_{i \in \mathbb{N}}$, onde $\varepsilon^{i} \in\{0,1\}$ é o tipo de $R^{i}(f)$ e $\pi^{i+1}=r_{\varepsilon^{i}}\left(\pi^{i}\right)$ é chamada combinatória de $f$.

Definição 2.2.2 (combinatória $k$-limitada). Seja $f:[0,1) \rightarrow[0,1)$ uma t.i.i. e $\gamma(f)=$ $\left\{\pi^{i}, \varepsilon^{i}\right\}_{i \in \mathbb{N}}$ sua combinatória. Nós dizemos que $\gamma(f)$ é $k$-limitado, se para todo $n \in \mathbb{N}$ e para todo $\alpha, \beta \in \mathcal{A}$, existem sequências $n \leq n_{0}<n_{1}<\ldots<n_{j+1} \leq n+k e$ $\alpha=\alpha_{0}, \alpha_{1}, \ldots, \alpha_{j+1}=\beta$, tal que para todo $0 \leq i \leq j$, as letras $\alpha_{i}$ e $\alpha_{i+1}$ são as letras vencedoras e perdedoras, respectivamente, da $n_{i}-$ ésima renormalização.

Observação 2.2.2. Para $d=2$ a definição acima é equivalente ao número de rotação de $f$ ser "tipo limitado".

Seja $n=n(\pi, f)$ o menor inteiro tal que $\varepsilon^{j} \neq \varepsilon$. A indução de Zorich é a aplicação definida por

$$
\hat{\mathcal{Z}}(f)=\hat{R}^{n}(f) .
$$

A renormalização de Zorich ou renormalização acelerada é definida como $\mathcal{Z}(f)=R^{n}(f)$.

\subsection{Classe de Rauzy}

Dadas duas permutações $\pi$ e $\pi^{\prime}$, nós dizemos que $\pi^{\prime}$ um sucessor de $\pi$ se existe $\varepsilon \in\{0,1\}$ tal que $r_{\varepsilon}(\pi)=\pi^{\prime}$. Qualquer par $\pi$ tem exatamente dois sucessores, correspondendo aos tipos $0 \mathrm{e}$ 1. Similarmente, cada $\pi^{\prime}$ é o sucessor de exatamente dois pares $\pi$. Note que $\pi$ irredutível se, e somente se, $\pi^{\prime}$ irredutível. Assim, esta relação define uma ordem parcial no conjunto das 
permutações irredutíveis $\Pi_{d}$, que pode ser representado como um grafo chamado de grafo de Rauzy. As componentes conexas deste grafo, nós chamamos classes de Rauzy.

Para $d=2$ existem duas possibilidades de permutações, mas só uma irredutível: $(2,1)$. O grafo de Rauzy se resume à Figura 2.3.

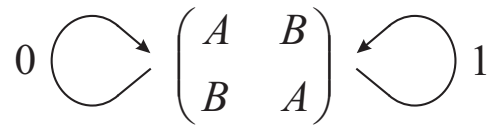

Figura 2.1: Classe de Rauzy para $d=2$.

Para $d=3$ existem seis possibilidades de permutação, mas apenas três delas são irredutíveis, a saber: $(2,3,1),(3,1,2),(3,2,1)$. Elas estão representadas na mesma classe de Rauzy. Ver Figura 2.3.

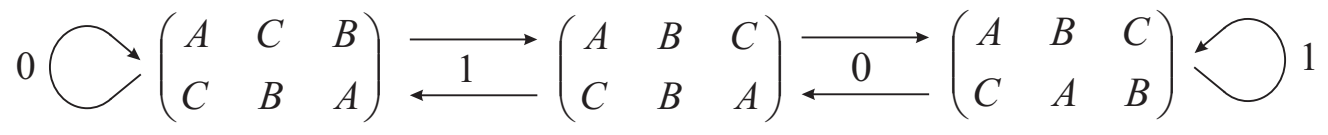

Figura 2.2: Classe de Rauzy para $d=3$.

Assim, só existe uma única classe de Rauzy para $d=3$.

Para $d=4$ existem 24 possibilidades de permutações, 13 das quais são irredutíveis:

$$
\begin{array}{lllll}
(4,3,2,1) & (4,1,3,2) & (3,1,4,2) & (4,2,1,3) & (2,4,3,1) \\
(3,2,4,1) & (2,4,1,3) & (4,2,3,1) & (4,1,2,3) & (4,3,1,2) \\
(3,4,1,2) & (2,3,4,1) & (3,4,2,1) & &
\end{array}
$$

A seguinte classe de Rauzy, com sete vértices, ocorre para os primeiros sete valores acima:

Os últimos seis valores ocorrem na classe de Rauzy apresentada na Figura 2.3. Assim, existem exatamente duas classes de Rauzy para $d=4$.

Seja $f$ uma t.i.i. sem conexão. Sua combinatória $\left\{\pi^{i}, \varepsilon^{i}\right\}_{i \in \mathbb{N}}$, pode ser identificada como uma caminho no diagrama de Rauzy, e será denotado por $\gamma(f)$. Nós dizemos que o caminho $\gamma(f)$ é completo se cada letra em $\mathcal{A}$ é vencedora de pelo menos uma vez; é $k$-completo se $\gamma(f)$ é a concatenção de $k$ caminhos completos. Um caminho infinito é dito ser $\infty$-completo se é a concatenação de infinitos caminhos completos.

Definição 2.3.1. Uma t.i.i. generalizada $f$ é irracional se não tem conexão e $\gamma(f)$ é $\infty$-completo. Nós chamamos $\gamma(f)$ de número de rotação de $f$.

Esta definição é motivada pela seguinte proposição.

Proposição 2.3.1. Sejam $f: J \rightarrow J$ uma t.i.i. generalizada sem conexão, e $T: I \rightarrow I$ uma t.i.i. standard sem conexão, tal que $\gamma(f)=\gamma(T)$ é $\infty$-completo. Então existe uma aplicação crescente, contínua e sobrejetiva $h: J \rightarrow I$ tal que

$$
f \circ h=h \circ g .
$$




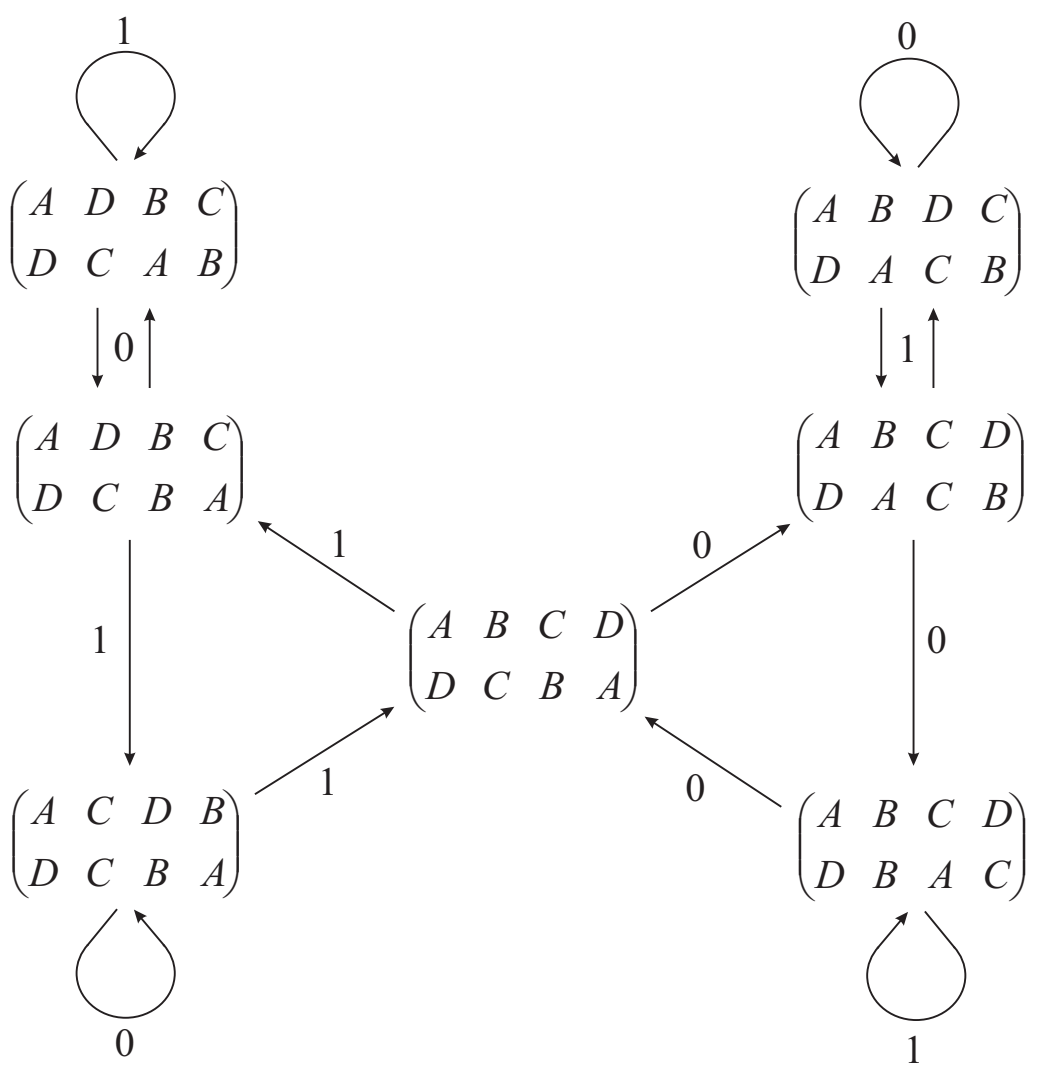

Figura 2.3: Classe de Rauzy para $d=4$.

Observação 2.3.1. A transformação $T$ não é unicamente determinada por $f$, mas as possíveis escolhas, são topologicamente conjugadas.

Demonstração. Sejam $\left(u_{f, 1}^{t}, \ldots, u_{f, d}^{t}\right),\left(u_{f, 1}^{b}, \ldots, u_{f, d}^{b}\right),\left(u_{T, 1}^{t}, \ldots, u_{T, d}^{t}\right)$ e $\left(u_{T, 1}^{b}, \ldots, u_{T, d}^{b}\right)$ as singularidades de $f, f^{-1}, g$ e $g^{-1}$, respectivamente. Seja

$$
S(f)=\left\{f^{m}\left(u_{f, i}^{b}\right), m \geq 0 \text { e } 1 \leq i \leq d\right\} \cup\left\{f^{-n}\left(u_{f, j}^{t}\right), n \geq 0 \text { e } 1 \leq j \leq d\right\}
$$

e

$$
S(T)=\left\{T^{m}\left(u_{T, i}^{b}\right), m \geq 0 \text { e } 1 \leq i \leq d\right\} \cup\left\{T^{-n}\left(u_{T, j}^{t}\right), n \geq 0 \text { e } 1 \leq j \leq d\right\} .
$$

Definamos $h: S(f) \rightarrow S(T)$ por

$$
\begin{gathered}
h\left(f^{-j}\left(u_{f, j}^{t}\right)\right)=T^{-j}\left(u_{T, j}^{t}\right), \text { se } j \geq 0, \\
h\left(f^{j}\left(u_{f, j}^{b}\right)\right)=T^{j}\left(u_{T, j}^{b}\right), \text { se } j \geq 0 .
\end{gathered}
$$

Como $\gamma(f)=\gamma(T)$ temos que $S(f)$ e $S(T)$ estão dispostos da mesma maneira e portanto $h$ é uma bijeção crescente sobre estes conjuntos. 


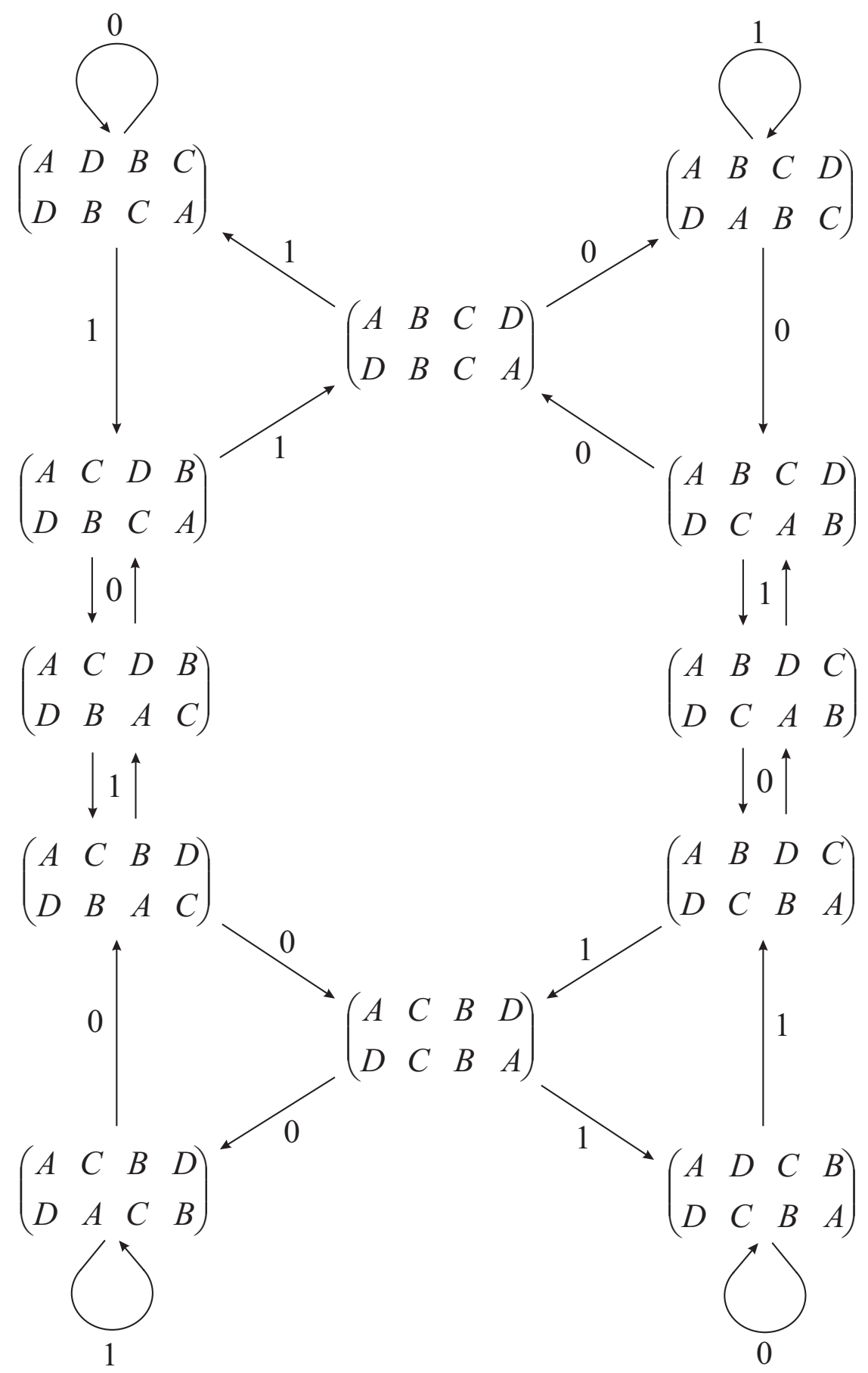

Figura 2.4: Classe de Rauzy para $d=4$.

Como $T$ é minimal, temos que $\overline{S(T)}=I$. Assim existe uma única aplicação crescente do intervalo $J$ no intervalo $I$ que prolonga $h$, e que com um certo abuso de notação ainda denotaremos por $h$. Este prolongamento é contínuo e sobrejetor. Por continuidade tem-se

$$
h \circ f=T \circ h .
$$




\subsection{Suspensão e Genus de t.i.i.'s.}

Seja $\pi$ uma permutação irredutível e $\lambda \in \mathbb{R}^{\mathcal{A}}$ o vetor comprimento. Denotamos por $T_{\pi}^{+}$o subconjunto de vetores $\tau=\left(\tau_{\alpha}\right)_{\alpha \in \mathcal{A}} \in \mathbb{R}^{\mathcal{A}}$ tais que

$$
\sum_{\pi_{0}(\alpha) \leq k} \tau_{\alpha}>0 \quad \text { e } \quad \sum_{\pi_{1}(\alpha) \leq k} \tau_{\alpha}<0
$$

para todo $1 \leq k \leq d-1$.

Claramente, $T_{\pi}^{+}$é um cone convexo. Nós dizemos que $\tau$ tem tipo 0 se $\sum_{\alpha \in \mathcal{A}} \tau_{\alpha}>0$ e tipo 1 se $\sum_{\alpha \in \mathcal{A}} \tau_{\alpha}<0$. Defina $\zeta_{\alpha}=\left(\lambda_{\alpha}, \tau_{\alpha}\right) \in \mathbb{R}^{2}$ e considere a curva fechada $\Gamma=\Gamma(\pi, \lambda, \tau)$ em $\mathbb{R}^{2}$ formada pela concatenação de

$$
\zeta_{\pi_{0}^{-1}(1)}, \zeta_{\pi_{0}^{-1}(2)}, \ldots, \zeta_{\pi_{0}^{-1}(d)},-\zeta_{\pi_{1}^{-1}(d)}, \ldots,-\zeta_{\pi_{1}^{-1}(1)}
$$

A superfície suspensão $M=M(\pi, \lambda, \tau)$ é a superfície obtida pela identificação dos lados paralelos, que são os lados marcados pela mesma letra do alfabeto $\mathcal{A}$. Seja $I \subset M$ o segmento horizontal de comprimento $\sum_{\alpha \in \alpha} \lambda_{\alpha}$ tendo a origem como ponto inicial, isto é

$$
I=\left[0, \sum_{\alpha \in \mathcal{A}} \lambda_{\alpha}\right) \times\{0\} .
$$

A transformação de intercâmbio de intervalos definida por $f=(\pi, \lambda)$ corresponde a aplicação de primeiro retorno em $I$ do fluxo vertical em $M$. De fato, considerando

$$
I_{\alpha}=\left[\sum_{\pi_{0}(\beta)<\pi_{0}(\alpha)} \lambda_{\beta}, \sum_{\pi_{0}(\beta) \leq \pi_{0}(\alpha)} \lambda_{\beta}\right) \times\{0\},
$$

e o segmento vertical começando em $(x, 0) \in I_{\alpha}$. Esse segmento vertical toca o lado representado por $\zeta_{\alpha}$ em algum ponto $(x, z)$. Este ponto é identificado com um ponto $\left(x^{\prime}, z^{\prime}\right)$ que está no lado representado por $-\zeta_{\alpha}$. Então temos que

$$
\begin{aligned}
& x^{\prime}=x-\sum_{\pi_{0}(\beta)<\pi_{0}(\alpha)} \lambda_{\beta}+\sum_{\pi_{1}(\beta)<\pi_{1}(\alpha)} \lambda_{\beta}=x+\omega_{\alpha} \\
& z^{\prime}=z-\sum_{\pi_{0}(\beta)<\pi_{0}(\alpha)} \tau_{\beta}+\sum_{\pi_{1}(\beta)<\pi_{1}(\alpha)} \tau_{\beta}=x-h_{\alpha},
\end{aligned}
$$

onde $h_{\alpha}>0$ é definido pela última equação. Movendo agora o ponto $\left(x^{\prime}, z^{\prime}\right)$ pelo segmento vertical, temos que o fluxo volta a interceptar $I$ no ponto $\left(x^{\prime}, 0\right)$. Isto mostra que se $f$ é uma t.i.i. standard, definida pelo par $(\pi, \lambda)$, a aplicação de primeiro retorno a $I$, coincide com $f$.

Vimos que dado um dado combinatorial $\pi$, nós podemos associar uma superfície compacta orientável $M$. Seja $g$ o genus desta superfície. O seguinte resultado pode ser encontrado em [Via08, Proposição 2.5]. 
Proposição 2.4.1. O genus da superfície de translação $M$ é constante em cada classe de Rauzy.

Denotaremos por $\Pi_{d}^{g}$ o conjunto das permutações irredutíveis que pertencem à classe de Rauzy de genus $g$.

Nós dizemos que uma t.i.i. $f: I \rightarrow I$ tem genus $g$ se o dado combinatorial, $\pi$, associado a $f$ pertence a $\Pi_{d}^{g}$.

Em [NR97] os autores dão uma caracterização para as t.i.i. de genus 1. Uma t.i.i. $f: I \rightarrow I$ tem genus 1 , se e somente se, $f$ possui no máximo duas descontinuidades. Por exemplo, para $d=2,3$ toda t.i.i. tem genus 1 .

O leitor pode notar que, para qualquer $d \geq 2$, existe $\pi \in \Pi_{d}^{1}$ tal que $f$ só possui uma descontinuidade, e neste caso, $f$ pode ser vista como um homeomorfismo do círculo. Por causa disto, alguns autores chamam a classe de Rauzy de genus 1 de classe de rotação. ([NR97].)

\subsection{Cociclo de Rauzy-Veech}

Seja $\pi \in \Pi_{d}^{g}$. Defina o operador linear $\Theta_{\pi, \varepsilon}: \mathbb{R}^{\mathcal{A}} \rightarrow \mathbb{R}^{\mathcal{A}}$ por

$$
\Theta_{\pi, \varepsilon}=\mathrm{Id}+E_{\alpha(1-\varepsilon) \alpha(\varepsilon)}
$$

onde $E_{\alpha \beta}$ é a matriz elementar cujo o único coeficiente não zero é igual a 1 na posição $\alpha \beta$.

Em particular, se $f=(\pi, \lambda)$ é uma t.i.i. standard renormalizável, com $\hat{R}(f)=f_{1}=$ $\left(\pi^{1}, \lambda^{1}\right)$, então

$$
\lambda^{1}={ }^{t} \Theta_{\pi, \varepsilon}^{-1}(\lambda) \quad \text { ou } \quad \lambda={ }^{t} \Theta_{\pi, \varepsilon}\left(\lambda^{1}\right),
$$

onde ${ }^{t} \Theta$ denota o operador adjunto de $\Theta$, isto é, o operador cuja matriz é a transposta de $\Theta$ e $\varepsilon \in\{0,1\}$ é o tipo de $f=(\pi, \lambda)$.

Se $f$ for uma transformação de intercâmbio de intervalos afim, nós chamamos de vetor inclinação de $f$ o vetor $\omega=\left(\omega_{\alpha}\right)_{\alpha \in \mathcal{A}}$ tal que $\omega_{\alpha}=\left.\ln D f\right|_{I_{\alpha}}$. Se $f$ é renormalizável, então nós podemos obter o vetor inclinação $\omega^{1}=\left(\omega_{\alpha}^{1}\right)_{\alpha \in \mathcal{A}}$ de $\hat{R} f$ da seguinte maneira:

$$
\Theta_{\pi, \varepsilon}(\omega)=\omega^{1}
$$

Se $f$ é infinitamente renormalizável então $\Theta_{\pi^{n}, \varepsilon^{n}}\left(\omega^{n}\right)=\omega^{n+1}$, isto é, a sequência $\omega^{n}=$ $\left(\omega_{\alpha}^{n}\right)_{\alpha \in \mathcal{A}}$ é uma órbita para o cociclo de Rauzy-Veech.

Outra interpretação para a matriz $\Theta_{\pi, \varepsilon}$ é a seguinte: Seja $f: I \rightarrow I$ uma transformação de intercâmbio de intervalos generalizadas sem conexão, com dado combinatorial $\pi^{0}$ e tipo $\varepsilon \in\{0,1\}$ e $R f: I^{1} \rightarrow I^{1}$ sua renormalização. Então

$$
\left(\Theta_{\pi, \varepsilon}\right)_{\alpha, \beta}=\sharp\left\{0 \leq i<r\left(I_{\alpha}^{1}\right): f^{i}\left(I_{\alpha}^{1}\right) \subset I_{\beta}\right\} \text { para todo } \alpha, \beta \in \mathcal{A},
$$


onde $r\left(I_{\alpha}^{1}\right)$ é o tempo de primeiro retorno do intervalo $I_{\alpha}^{1}$ ao intervalo $I^{1}$ sob iterados de $f$.

Assim se $\left\{\pi^{i}, \varepsilon^{i}\right\}_{i \in \mathbb{N}}$ é a combinatória de $f$ então definindo

$$
\Theta_{0, n-1}:=\Theta_{\pi^{n-1}, \varepsilon^{n-1}} \cdots \Theta_{\pi^{1}, \varepsilon^{1}} \cdot \Theta_{\pi^{0}, \varepsilon^{0}},
$$

temos, por indução em $n$, que

$$
\left(\Theta_{0, n-1}\right)_{\alpha, \beta}=\sharp\left\{0 \leq j<r\left(I_{\alpha}^{n}\right): f^{j}\left(I_{\alpha}^{n}\right) \subset I_{\beta}\right\} \text { para todo } \alpha, \beta \in \mathcal{A},
$$

onde $r\left(I_{\alpha}^{n}\right)$ é o tempo de primeiro retorno do intervalo $I_{\alpha}^{n}$ ao intervalo $I^{n}$ sob iterados de $f$ [Via08, Proposição 6.14].

O cociclo de Rauzy-Veech são as funções

$$
\Theta_{\varepsilon}: \Pi_{d}^{g} \times \mathbb{R}^{\mathcal{A}} \rightarrow \Pi_{d}^{g} \times \mathbb{R}^{\mathcal{A}}
$$

$\operatorname{com} \varepsilon \in\{0,1\}$, definidas por $\Theta_{\varepsilon}(\pi, v)=\left(r_{\varepsilon}(\pi), \Theta_{\pi, \epsilon} v\right)$.

Seja $\pi$ um dado combinatorial de uma t.i.i. e seja $\lambda=\left(\lambda_{\alpha}\right)_{\alpha \in \mathcal{A}}$ um vetor em $\mathbb{R}^{\mathcal{A}}$. Defina $\delta=\left(\delta_{\alpha}\right)_{\alpha \in \mathcal{A}}$ como

$$
\delta_{\alpha}=\sum_{\pi_{1}(\beta)<\pi_{1}(\alpha)} \lambda_{\beta}-\sum_{\pi_{0}(\beta)<\pi_{0}(\alpha)} \lambda_{\beta}
$$

Note que $\Omega_{\pi}(\lambda)=\delta$, onde $\Omega_{\pi}$ é a matriz anti-simétrica dada por

$$
\Omega_{\alpha, \beta}= \begin{cases}+1 & \text { se } \pi_{1}(\alpha)>\pi_{1}(\beta) \text { and } \pi_{0}(\alpha)<\pi_{0}(\beta) \\ -1 & \text { se } \pi_{1}(\alpha)<\pi_{1}(\beta) \text { and } \pi_{0}(\alpha)>\pi_{0}(\beta) \\ 0 & \text { caso contrário. }\end{cases}
$$

Se $\pi \in \Pi_{d}^{g}$ então $\operatorname{dim} \operatorname{Ker} \Omega_{\pi}=d-2 g$, e assim $\operatorname{dim} \operatorname{Im} \Omega_{\pi}=2 g$, [Via08, Lema 2.10]. Sabemos também que pelo fato de $\Omega_{\pi}$ ser anti-simétrica

$$
\operatorname{Ker} \Omega_{\pi} \perp \operatorname{Im} \Omega_{\pi}:=H_{\pi} .
$$

A seguinte relação será usada várias vezes neste trabalho.

Lema 2.5.1. Se $\hat{R}(\pi, \lambda)=\left(\pi^{1}, \lambda^{1}\right)$ ent $\tilde{a} o \Theta_{\pi, \varepsilon} \Omega_{\pi}^{t} \Theta_{\pi, \varepsilon}=\Omega_{\pi^{1}}$.

Demonstração. A demonstração pode ser encntrada em [Via08, Lema 1.29].

Corolário 2.5.1. O subespaços Ker $\Omega_{\pi}$ e $\operatorname{Im} \Omega_{\pi}$ são invariantes por ${ }^{t} \Theta_{\pi, \varepsilon}^{-1}$ e $\Theta_{\pi, \varepsilon}$, respectivamente. Ou seja

$$
{ }^{t} \Theta_{\pi, \varepsilon}^{-1}\left(\operatorname{Ker} \Omega_{\pi}\right)=\operatorname{Ker} \Omega_{\pi^{1}} \text { e } \Theta_{\pi, \varepsilon}\left(\operatorname{Im} \Omega_{\pi}\right)=\operatorname{Im} \Omega_{\pi^{1}} .
$$

O Corolário (2.5.1) pode ser resumido no seguinte digrama: 


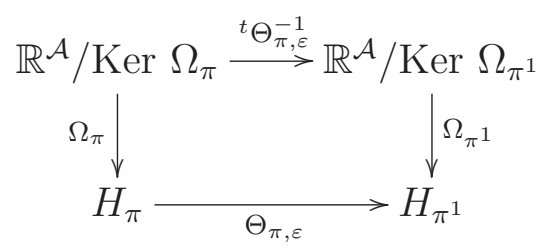

É possível calcular explicitamente uma base para Ker $\Omega_{\pi}$. É claro que essa base só depende da permutação $\pi$. Para isto, defina uma nova permutação $\sigma:\{0,1, \ldots, d\} \rightarrow$ $\{0,1, \ldots, d\}$ por

$$
\sigma(j)= \begin{cases}p^{-1}(1)-1 & \text { se } j=0 \\ d & \text { se } j=p^{-1}(d) \\ p^{-1}(p(j)+1)-1 & \text { caso contrário }\end{cases}
$$

onde $p$ é a monodromia invariante definida em (2.1).

Para cada órbita $\mathcal{O}$ de $\sigma$ não contendo zero, e para cada $1 \leq j \leq d$, definimos

$$
\lambda(\mathcal{O})_{j}=\chi_{\mathcal{O}}(j)-\chi_{\mathcal{O}}(j-1)= \begin{cases}1, & \text { se } j \in \mathcal{O} \text { mas } j-1 \notin \mathcal{O} \\ -1, & \text { se } j \notin \mathcal{O} \text { mas } j-1 \in \mathcal{O} \\ 0, & \text { caso contrário. }\end{cases}
$$

Segue de [Via08, Lema 2.11] que o conjunto

$$
\{\lambda(\mathcal{O}): \mathcal{O} \text { é uma órbita de } \sigma \text { não contendo } 0\}
$$

é uma base de Ker $\Omega_{\pi}$. Portanto, desde que $\Omega_{\pi}$ é antisimétrica, a $\operatorname{Im} \Omega_{\pi}$ é o complemento ortogonal deste conjunto.

Pelo Corolário 2.5.1, temos que ${ }^{t} \Theta_{\pi, \varepsilon}^{-1}\left(\operatorname{Ker} \Omega_{\pi}\right)=\operatorname{Ker} \Omega_{\pi^{\prime}}$. O próximo Lema, cuja demosntração pode ser encontrada em [Via08, Lema 2.16], nos dá uma relação entre as bases de $\operatorname{Ker} \Omega_{\pi}$ e $\operatorname{Ker} \Omega_{\pi^{\prime}}$.

Lema 2.5.2. Dados $\pi$ e $\varepsilon \in\{0,1\}$, seja $\pi^{\prime}=r_{\varepsilon}(\pi)$ e $\sigma, \sigma^{\prime}$ as permutações de $\{0,1, \ldots, d\}$ correspondente a $\pi$ e $\pi^{\prime}$, respectivamente. Então para cada $\sigma$-órbita $\mathcal{O}$ não contendo o 0, existe uma $\sigma^{\prime}$-órbita, $\mathcal{O}^{\prime}$, não contendo 0 , tal que ${ }^{t} \Theta_{\pi, \varepsilon} \lambda(\mathcal{O})=\lambda\left(\mathcal{O}^{\prime}\right)$.

\subsection{Relação entre t.i.i. de genus um e homeomorfismos do círculo}

Seja $\mathcal{B}_{k}^{r}$ o conjunto das transformações de intercâmbio de intervalos generalizadas $f$, tais que 
(i) Para cada $\alpha \in \mathcal{A}$ nós temos que $\left.f\right|_{I_{\alpha}}$ é um difeomorfismo de classe $C^{r}$;

(ii) $f$ tem combinatória $k$-limitada;

(iii) $f$ tem genus um;

(iv) $f$ não tem conexão.

Se $f \in \mathcal{B}_{k}^{r}$ tem somente uma descontinuidade, então se identificarmos o ponto final com o ponto inicial do domínio $[0,1)$, obtemos um homemomorfismo do círculo que é $C^{r}$ por pedaços. Assim, se $r \geq 2$, nós poderemos aplicar o Teorema de Denjoy e mostrar que $f$ não possui intervalos errantes. Esse fato é a principal diferença entre transformações de intercâmbio de intervalos de genus um com as transformações de intercâmbio de intervaos de genus maior do que um. Nesta seção nós estudaremos a relação entre transformações de intercâmbio de intervalos generalizada de genus um e homeomorfismos do círculo suave por pedaços.

Por questão de simplicidade, assumimos que $f$ tem somente uma descontinuidade. Então $f$ pode ser vista como uma transformação de intercâmbio de intervalos generalizada em $\mathcal{B}_{k}^{0+1}$ com dois intervalos. De fato, seja $I_{\alpha}=\left[c_{\alpha}, d_{\alpha}\right)$, onde $d_{\alpha}$ é o único ponto de descontinuidade de $f$. Então definimos

$$
\begin{aligned}
J_{A} & :=\bigcup_{\pi_{0}(\beta) \leq \pi_{0}(\alpha)} I_{\beta}, \\
J_{B} & :=\bigcup_{\pi_{0}(\beta)>\pi_{0}(\alpha)} I_{\beta} .
\end{aligned}
$$

Então $\left(f,\{A, B\},\left\{J_{A}, J_{B}\right\}\right)$ é uma transformação de intercâmbio de intervalos generalizada com dois intervalos. Assim, nós podemos renormalizar $f$ como uma t.i.i. generalizada de $d$ intervalos ou como uma t.i.i. generalizada de dois intervalos, que denotaremos por $R_{d}(f)$ e $R_{2}(f)$, respectivamente. Se nós enxergarmos $f$ como um homeomorfismo do círculo, nós podemos renormalizá-lo como tal. Essa renormalização nós denotaremos por $R_{\text {rot }}(f)$. Essa renormalização coincide com a renormalização de Zorich de $R_{2}(f)$, ou seja

$$
R_{\mathrm{rot}}(f)=R_{2}^{n_{i}}(f),
$$

onde $n_{0}=0$ e $n_{i}$ é o primeiro iterado $n>n_{i-1}$ cujo tipo é diferente de $R_{2}^{n_{i}}(f)$.

O próximo resultado nos dá uma relação entre $R_{d}(f)$ e $R_{2}(f)$.

Proposição 2.6.1. Seja $f$ uma t.i.i. generalizada de genus um, sem conexão, com $d$ intervalos, tendo somente uma descontinuidade. Denote por $\alpha_{0}, \alpha_{1} \in \mathcal{A}$ tal que $\pi_{1}\left(\alpha_{1}\right)=1$ e $\pi_{0}\left(\alpha_{0}\right)=1$. Então temos duas possibilidades 
(A) $S e$

$$
\cup_{\pi_{1}(\beta) \geq \pi_{1}\left(\alpha_{0}\right)} f\left(I_{\beta}\right) \subset \cup_{\pi_{0}(\beta) \geq \pi_{0}\left(\alpha_{1}\right)} I_{\beta},
$$

então $R_{2}(f)$ é bem definido, tem tipo 0 e $R_{2}(f)=R_{d}^{n}(f)$, onde $n-1$ é tal que a letra $\alpha_{0}$ perde pela primeira vez.

(B) $S e$

$$
\cup_{\pi_{0}(\beta) \geq \pi_{0}\left(\alpha_{1}\right)} I_{\beta} \subset \cup_{\pi_{1}(\beta) \geq \pi_{1}\left(\alpha_{0}\right)} f\left(I_{\beta}\right),
$$

então $R_{2}(f)$ é bem definido, tem tipo 1 e $R_{2}(f)=R_{d}^{n}(f)$, onde $n-1$ é tal que a letra $\alpha_{1}$ perde pela primeira vez.

Demonstração. Provaremos a afirmação A. A prova da afirmação B é similar. Usando a notação definida (2.1), temos que o algoritmo de Rauzy-Veech é dado por

$$
(p(1), p(2), \ldots, p(s), \ldots, p(d)) \stackrel{0}{\rightarrow}(p(2), \ldots, p(1), p(s), \ldots, p(d)),
$$

onde $s$ é tal que $p(s)=1$, e

$$
(p(1), \ldots, p(r), \ldots, p(d)) \stackrel{1}{\rightarrow}(p(1), \ldots, p(r), p(d), \ldots),
$$

onde $r$ é tal que $p(r)=d$.

Como por hipótese $f$ tem somente uma descontinuidade, temos que $p=(k \ldots d 1 \ldots k-$ $1)$, onde $\pi_{1}\left(\alpha_{0}\right)=k$.

Afirmamos que iterando o algoritmo $N=s+r \leq n-1$ vezes, obtemos

$$
p^{N}=(k+s, \ldots, d, k-r, \ldots, k+s-1,1, \ldots, k-r-1),
$$

onde $0 \leq s \leq d-k$ e $0 \leq r \leq k-1$ são tais que

$$
s=\#\left\{\varepsilon^{m}=0: 0 \leq m \leq N\right\} \text { e } r=\#\left\{\varepsilon^{m}=1: 0 \leq m \leq N\right\} .
$$

Para $N=1$ a afirmação é verdadeira, pois $s=0$ e $r=1$ ou $s=1$ e $r=0$. Assuma que a fórmula (2.17) vale para $N-1$. Então por (2.15) e (2.16) a afirmação vale para $N$.

Pela definição de $n$, temos que $\pi_{0}^{n-1}\left(\alpha_{0}\right)=1$ e $\pi_{1}^{n-1}\left(\alpha_{0}\right)=d$. Portanto $p^{n-1}(1)=d$ e

$$
p=p^{0}=(k \ldots d 1 \ldots k-1) \stackrel{\varepsilon^{0}}{\rightarrow} \ldots \stackrel{\varepsilon^{n-2}}{\rightarrow} p^{n-1}=(d k-r \ldots d-11 \ldots k-r-1) .
$$

Novamente, a definição de $n$ nos dá que $\varepsilon^{n-1}=0$. Logo

$$
p^{n}=(k-r \ldots d-1 d 1 \ldots k-r-1),
$$

o que completa a prova. 
Corolário 2.6.1. Seja $f$ uma t.i.i. generalizada sem conexão, com d intervalos e somente uma descontinuidade. Então existe sequência $m_{i}<m_{i+1}$ tal que $m_{i+1}-m_{i}<d$ $e$

$$
R_{2}(f)^{i}=R_{d}^{m_{i}}(f)
$$

Demonstração. A existência da sequência $\left\{m_{i}\right\}_{i \in \mathbb{N}}$ é garantido pela Proposição 2.6.1. Pela equação (2.17) temos que o tempo máximo para que $R_{d}^{n}(f)$ possua somente uma descontinuidade é $d-(r+s)$, que é no máximo $d-1$.

Corolário 2.6.2. Seja $f \in \mathcal{B}_{k}$. Então para todo $i \geq 0$

$$
R_{\mathrm{rot}}^{i}(f)=R_{2}^{k_{i}}(f)=R^{n_{k_{i}}}(f) .
$$

Demonstração. A primeira igualdade segue da definição de $R_{\text {rot }}$ como já comentado anteriormente. A segunda igualdade, segue do Corolário 2.6.1.

\subsection{Estimativas tipo Denjoy}

Definição 2.7.1. $f: S^{1} \rightarrow S^{1}$ é dito ser um homeomorfismo de classe $P$, se o seu levantamento $\tilde{f}$ é diferenciável, exceto para uma quantidade enumerável de pontos, admitindo as derivadas laterais nesses pontos. A derivada de $\tilde{f}$, que denotaremos por, $h: \mathbb{R} \rightarrow \mathbb{R}$, é uma função $\mathbb{Z}$-periódica e de variação limitada em $[0,1]$.

É claro que se $f \in \mathcal{B}_{k}^{1}$ tem somente uma descontinuidade então $f$ é um homeomorfismo do círculo de classe P.

Enunciaremos dois lemas, cujas demonstrações podem ser encontradas em [Her79, Proposição 1.1, VI] e [Her79, Teorema 3.1, VI], respectivamente.

Lema 2.7.1. Seja $f$ um homeomorfismo de classe P. Então

$$
\int_{S^{1}} \log h d \mu=0
$$

onde $\mu$ denota a única medida de probabilidade invariante por $f$.

Lema 2.7.2 (Desigualdade de Denjoy-Koksma). Seja $f$ um homeomorfismo de classe $P$, e $\frac{p}{q}$ uma aproximação racional de $\rho(f)$. Seja $\varphi: S^{1} \rightarrow \mathbb{R}$ uma função de variação limitada (não necessariamente contínua), e seja $\mu$ uma medida de probabilidade sobre $S^{1}$, invariante por $f$. Então para todo $x \in S^{1}$,

$$
\left|\sum_{i=0}^{q-1} \varphi \circ f^{i}(x)-q \int_{S^{1}} \varphi d \mu\right| \leq \operatorname{Var}(\varphi)
$$


Proposição 2.7.1 (Desigualdade de Denjoy). Seja $f$ um homoemorfismo de classe P, e $\frac{p_{n}}{q_{n}}$ a aproximação de $\rho(f)$ por frações contínuas. Exceto, sobre um conjunto enumerável de pontos,

$$
e^{-V} \leq D f^{ \pm q_{n}} \leq e^{V}, \text { onde } V=\operatorname{Var}(\log D f)
$$

Demonstração. Exceto sobre um conjunto enumerável de pontos temos

$$
\log D f^{n}=\sum_{i=0}^{n-1} \log D f \circ f^{i}=\sum_{i=0}^{n-1} \log h \circ f^{i} .
$$

Pela Desigualdade de Denjoy-Koksma (Lema 2.7.2), temos que

$$
\sup _{x \in S^{1}}\left|\sum_{i=0}^{q_{n}-1} \log h \circ f^{i}(x)-q_{n} \int_{S^{1}} \log h d \mu\right| \leq \operatorname{Var}(\log h)=V .
$$

Pela proposição 2.7.1, temos que

$$
\int_{S^{1}} \log D f d \mu=\int_{S^{1}} \log h d \mu=0
$$

Assim,

$$
-V \leq \log D f^{q_{n}} \leq V \Rightarrow e^{-V} \leq D f^{q_{n}} \leq e^{V}
$$

Para provar a desigualdade para $D f^{-q_{n}}$, basta observar que $D f^{-q_{n}}=$ $\frac{1}{D f^{q_{n}} \circ f^{-q_{n}}}$.

Proposição 2.7.2. Sejam $f_{1}, f_{2} \in \mathcal{B}_{k}^{2}$ tais que a combinatória de $f_{1}$ é igual a combinatória de $f_{2}$. Então $f_{1}$ e $f_{2}$ são conjugadas.

Demonstração. Pela Proposição 2.7.1 temos que $f_{i}$ não possuem intervalos errantes, $i=1,2$. Assim pela Proposição 2.3.1 temos que $f_{i}$ é conjugada a uma t.i.i. standard $T_{i}, i=1,2$. Como a combinatória de $T_{1}$ é igual a combinatória de $T_{2}$, aplicando novamente a Proposição 2.3.1 temos que $T_{1}$ e $T_{2}$ são conjugadas e portanto $f_{1}$ e $f_{2}$ são conjugadas. 


$\frac{10}{3}$

\section{A Não-Linearidade e}

\section{Difeomorfismos de classe $C^{2+\nu}$}

Neste capítulo, nós descreveremos alguns resultados que serão utilizados no restante do texto, sobre o papel da função não-linearidade nos difeomorfismos de classe $C^{2+\nu}$. Em todo este capítulo, estaremos sob a suposição de que para qualquer difeomorfismo $f:[a, b] \rightarrow \mathbb{R}, D f(x) \geq c>0$ para todo $x \in[a, b]$. Em particular, o difeomorfismo $f$, preserva orientação. Os resultados aqui obtidos são independentes, no sentido de que não usaremos nenhum fato a cerca de transformações de intercâmbio de intervalos, bem como difeomorfismos do círculo.

Dada um difeomorfismo $f:[a, b] \rightarrow \mathbb{R}$ de classe $C^{2}$, nós definimos a não-linearidade de $f$ como a função $n_{f}:[a, b] \rightarrow \mathbb{R}$ dada por

$$
n_{f}(x)=\frac{D^{2} f(x)}{D f(x)}=D \ln D f(x) .
$$

A não-linearidade média de $f$, que denotaremos por $N_{f}$, é o número real definido por

$$
N_{f}=\int_{a}^{b} n_{f}(s) d s .
$$


Da equação (3.1) segue que

$$
n_{f \circ g}(x)=n_{f}(g(x))+n_{g}(x) .
$$

Proposição 3.0.3. Seja $f:[0,1] \rightarrow[0,1]$ uma difeomorfismo de classe $C^{2}$, tal que $f(0)=0$ e $f(1)=1$. Então para todo $x \in[0,1]$

$$
f(x)=\frac{\int_{0}^{x} e^{\int_{0}^{t} n_{f}(y) d y} d t}{\int_{0}^{1} e^{\int_{0}^{t} n_{f}(y) d y} d t}
$$

Demonstração. Como $n_{f}(y)=D \ln D f(y)$ nós temos que

$$
\int_{0}^{t} n_{f}(y) d y=\int_{0}^{t} D \ln D f(y) d y=\ln D f(t)-\ln D f(0)=\ln \frac{D f(t)}{D f(0)},
$$

e portanto,

$$
D f(t)=D f(0) \cdot e^{\int_{0}^{t} n_{f}(y) d y}
$$

Integrando novamente, obtemos

$$
f(x)=f(x)-f(0)=\int_{0}^{x} D f(t) d t=D f(0) \cdot \int_{0}^{x} e^{\int_{0}^{t} n_{f}(y) d y} d t .
$$

Usando o fato de que $f(1)=1$, conseguimos calcular o valor de $D f(0)$, a saber

$$
1=f(1)=D f(0) \cdot \int_{0}^{1} e^{\int_{0}^{t} n_{f}(y) d y} d t \Rightarrow D f(0)=\frac{1}{e^{\int_{0}^{t} n_{f}(y) d y} d t} .
$$

E portanto o resultado está mostrado.

Nós dizemos que uma função $f:[a, b] \rightarrow \mathbb{R}$ é de classe $C^{2+\nu}, \nu>0$, se $f$ é uma função de classe $C^{2}$, e $D^{2} f$ é $\nu$-hölder, ou seja, existe uma constante $C=C(f)>0$, tal que

$$
\left|D^{2} f(x)-D^{2} f(y)\right| \leq C \cdot|x-y|^{\nu}, \text { para todo } x, y \in[a, b] .
$$

Seja $f:[a, b] \rightarrow[f(a), f(b)]$ um difeomorfismo de classe $C^{2+\nu}$. Visto que $D f$ é limitada inferiormente, temos que $n_{f}$ é $\nu$-hölder, ou seja

$$
\left|n_{f}(x)-n_{f}(y)\right| \leq C_{0} \cdot|x-y|^{\nu}, \text { para todo } x, y \in[a, b]
$$

É fácil ver que se 


$$
n_{\mathrm{Z}_{[a, b]} f}(x)=(b-a) \cdot n_{f}(a+x(b-a)),
$$

e que $N_{f}=N_{\mathrm{Z}_{[a, b]} f}$, onde $\mathrm{Z}_{[a, b]}$ é o Zoom de $f$ em $[a, b]$.

Quando não houver confusão sobre qual intervalo nós estamos fazendo o zoom, nós escreveremos $\mathrm{Zf}$ para denotar $\mathrm{Z}_{[a, b]} f$. Desde que $D f$ é limitada inferiormente, temos que existe uma constante $C_{1}=C_{1}(f)>0$ tal que

$$
\left|n_{f}\right|_{C^{0}}:=\sup _{x \in[a, b]}\left\{\left|n_{f}(x)\right|\right\} \leq C_{1} .
$$

Lema 3.0.3. Seja $f:[a, b] \rightarrow[f(a), f(b)]$ um difeomorfismo de classe $C^{2+\nu}$. Então

$$
\left|n_{\mathrm{Z} f}\right|_{C^{0}} \leq C_{1} \cdot \delta \text { e }\left|n_{\mathrm{Z} f}(x)-n_{\mathrm{Z} f}(y)\right| \leq C_{0} \cdot \delta^{1+\nu},
$$

onde $\delta=b-a$.

Demonstração. Pela equação (3.4), nós obtemos diretamente a primeira desigualdade, e obtemos também que

$$
\begin{aligned}
\left|n_{\mathrm{Z} f}(x)-n_{\mathrm{Z} f}(y)\right| & =(b-a) \cdot\left|n_{f}(a+x(b-a))-n_{f}(a+y(b-a))\right| \\
& \leq C_{0} \cdot \delta \cdot \mid\left(a+x(b-a)-a+\left.y(b-a)\right|^{\nu}\right. \\
& \leq C_{0} \cdot \delta \cdot|x-y|^{\nu} \\
& \leq C_{0} \cdot \delta^{1+\nu} .
\end{aligned}
$$

\subsection{A Transformação de Möbius}

Uma transformação de Möbius $M: \mathbb{C} \cup\{\infty\} \rightarrow \mathbb{C} \cup\{\infty\}$ é uma função racional da forma

$$
M(z)=\frac{a z+b}{c z+d}
$$

onde $a, b, c$ e $d$ são números complexos e $a d-b c \neq 0$. A restrição $a d-b c \neq 0$ é essencial, pois caso contrário

$$
M^{\prime}(z)=\frac{a d-b c}{(c z+d)^{2}}=0 \text { para todo } z .
$$

A aplicação $M$ é bijetora e analítica. 
Neste trabalho, em particular, nós estamos interessados nas transformações Möbius $M:[0,1] \rightarrow[0,1]$ tal que $M(0)=0$ e $M(1)=1$. Nesse caso, $a, b, c, d \in \mathbb{R}$. A condição $M(0)=0$ implica que $b=0$ e a condição $M(1)=1$ implica que $a=c+d$. Queremos também que $M$ preserve orientação, o que é equivalente a $c+d$ e $d$ terem o mesmo sinal.

A função não-linearidade de $M$ é dada por

$$
n_{M}(x)=\frac{-2 c}{c x+d}
$$

e a não-linearidade média é o número

$$
N_{M}=2 \ln \frac{d}{c+d} .
$$

Em vista disso, a única transformação de Möbius $M_{N}:[0,1] \rightarrow[0,1]$ tal que $M_{N}(0)=0$ e $M_{N}(1)=1$ e cuja não-linearidade média é $N$ é dada por

$$
M_{N}(x)=\frac{x e^{\frac{-N}{2}}}{1+x\left(e^{\frac{-N}{2}}-1\right)} .
$$

Nosso próximo resultado nos dá uma estimativa entre o zoom de um difeomorfismo $f$, de classe $C^{2+\nu}$ e a transformação de Möbius cuja a não-linearidade média é $N_{f}$.

Proposição 3.1.1. Seja $f:[a, b] \rightarrow[f(a), f(b)]$ um difeomorfismo de classe $C^{2+\nu}$, cuja não-linearidade média é $N=N_{f}$, e Zf o zoom de $f$ em $[a, b]$. Então

$$
\mathrm{d}_{C^{2}}\left(\mathrm{Z} f, M_{N}\right)=\mathrm{O}\left(\delta^{1+\nu}\right)
$$

onde $\mathrm{d}_{C^{2}}$ denota a distância na topologia $C^{2}$ e $\delta=b-a$.

Antes de provar a proposição suponhamos que a função não-linearidade de $f$, e portanto de Z $f$, é constante e igual a $N$, ou seja, $n_{\mathrm{Z} f}(x)=N$ para todo $x \in[0,1]$. Pela equação (3.3) temos que

$$
\mathrm{Z} f(x)=\frac{\int_{0}^{x} e^{N t} d t}{\int_{0}^{1} e^{N t} d t}=\frac{e^{N x}-1}{e^{N}-1}
$$

E portanto,

$$
D Z f(x)=\frac{N e^{N x}}{e^{N}-1} \text { e } D^{2} Z f(x)=\frac{N^{2} e^{N x}}{e^{N}-1} .
$$

De (3.5) temos que 


$$
D M_{N}(x)=\frac{e^{-\frac{N}{2}}}{\left[1+x\left(e^{-\frac{N}{2}-1}\right)\right]^{2}} \text { e } D^{2} M_{N}=\frac{-2 e^{-\frac{N}{2}}\left(e^{-\frac{N}{2}}-1\right)}{\left[1+x\left(e^{-\frac{N}{2}-1}\right)\right]^{3}} .
$$

Então temos o seguinte Lema:

Lema 3.1.1. Seja $f_{N}:[a, b] \rightarrow\left[f_{N}(a), f_{N}(b)\right]$ um difeomorfismo de classe $C^{2+\nu}$, tal que a função não-linearidade de $\mathrm{Z} f_{N}$ seja constante igual a N. Então

$$
\mathrm{d}_{C^{2}}\left(\mathrm{Z} f_{N}, M_{N}\right)=\mathrm{O}\left(\delta^{2}\right)
$$

Demonstração. Na prova, nós usamos a expansão em série de Taylor das seguintes funções:

$$
\begin{gathered}
e^{x}=\sum_{n=0}^{\infty} \frac{x^{n}}{n !}=\sum_{n=0}^{k-1} \frac{x^{n}}{n !}+\mathrm{O}\left(x^{k}\right), \text { para todo } x \in \mathbb{R} \text { e } \\
\frac{1}{1-x}=\sum_{n=0}^{\infty} x^{n}=\sum_{n=0}^{k-1} x^{n}+\mathrm{O}\left(x^{k}\right), \text { para todo }|x|<1 .
\end{gathered}
$$

Assim,

$$
\begin{aligned}
\left|\mathrm{Z} f_{N}(x)-M_{N}(x)\right| & =\left|\frac{e^{N x}-1}{e^{N}-1}-\frac{x e^{\frac{-N}{2}}}{1+x\left(e^{\frac{-N}{2}}-1\right)}\right| \\
\leq & \left|\frac{N x+\frac{N^{2} x^{2}}{2}+\mathrm{O}\left(N^{3}\right)}{N+\frac{N^{2}}{2}+\mathrm{O}\left(N^{3}\right)}-\frac{x\left(1-\frac{N}{2}+\mathrm{O}\left(N^{2}\right)\right)}{1+x\left(-\frac{N}{2}+\mathrm{O}\left(N^{2}\right)\right)}\right| \\
\leq & \left|\frac{N x\left(1+\frac{N x}{2}+\mathrm{O}\left(N^{2}\right)\right)}{N\left(1+\frac{N}{2}+\mathrm{O}\left(N^{2}\right)\right)}-x\left(1-\frac{N}{2}+\mathrm{O}\left(N^{2}\right)\right)\left(1+\frac{N x}{2}+\mathrm{O}\left(N^{2}\right)\right)\right| \\
\leq & \left|x\left(1+\frac{N x}{2}\right)\left(1-\frac{N}{2}\right)+\mathrm{O}\left(N^{2}\right)-x\left(1+\frac{N x}{2}\right)\left(1-\frac{N}{2}\right)+\mathrm{O}\left(N^{2}\right)\right| \\
\leq & \mathrm{O}\left(N^{2}\right) \cdot \\
\left|D Z f_{N}(x)-D M_{N}(x)\right| & =\left|\frac{N e^{N x}}{e^{N}-1}-\frac{e^{\frac{-N}{2}}}{\left[1+x\left(e^{\frac{-N}{2}}-1\right)\right]^{2}}\right| \\
& \leq\left|\frac{N\left(1+N x+\mathrm{O}\left(N^{2}\right)\right)}{N\left(1+\frac{N}{2}+\mathrm{O}\left(N^{2}\right)\right)}-e^{-\frac{N}{2}}\left(1+\frac{N x}{2}+\mathrm{O}\left(N^{2}\right)\right)^{2}\right| \\
& \leq\left|(1+N x)\left(1-\frac{N}{2}\right)+\mathrm{O}\left(N^{2}\right)-e^{-\frac{N}{2}}\left[\left(1+\frac{N x}{2}\right)^{2}+\mathrm{O}\left(N^{2}\right)\right]\right| \\
& \leq \mid \mathrm{O}\left(N^{2}\right) . \\
& \leq \frac{N}{2}+N x+\mathrm{O}\left(N^{2}\right)-\left(1-\frac{N}{2}+O\left(N^{2}\right)\right)\left(1+N x+\mathrm{O}\left(N^{2}\right)\right) \mid
\end{aligned}
$$




$$
\begin{aligned}
\left|D^{2} \mathrm{Z} f_{N}(x)-D^{2} M_{N}(x)\right| & =\left|\frac{N^{2} e^{N x}}{e^{N}-1}-\frac{-2 e^{\frac{-N}{2}}\left(e^{-\frac{N}{2}}-1\right)}{\left[1+x\left(e^{\frac{-N}{2}}-1\right)\right]^{3}}\right| \\
& \leq\left|N+\mathrm{O}\left(N^{2}\right)-\left(N+\mathrm{O}\left(N^{2}\right)\right)\left(1+\frac{N x}{2}+\mathrm{O}\left(N^{2}\right)\right)^{3}\right| \\
& \leq \mathrm{O}\left(N^{2}\right) .
\end{aligned}
$$

Pelo Lema 3.0.3, temos que $\mathrm{O}(\delta)=\mathrm{O}(N)$. E o resultado está mostrado.

Demonstração. Proposição 3.1.1: Seja $N=\int_{0}^{1} n_{\mathrm{Z} f}(s) d s$. Então

$$
\mathrm{d}_{C^{2}}\left(\mathrm{Z} f, M_{N}\right) \leq \mathrm{d}_{C^{2}}\left(\mathrm{Zf}, \mathrm{Z} f_{N}\right)+\mathrm{d}_{C^{2}}\left(\mathrm{Z} f_{N}, M_{N}\right) .
$$

Em virtude do Lema 3.1.1, só precisaremos estimar a primeira parcela do lado direito da desigualdade acima. Agora, note que $N=\int_{0}^{1} n_{\mathrm{Zf} f}(s) d s=n_{\mathrm{Zf}}(\theta)$, para algum $\theta \in[0,1]$. Assim pelo Lema 3.0.3

$$
\left|n_{\mathrm{Z} f}(x)-N\right|=\left|n_{\mathrm{Z} f}(x)-n_{\mathrm{Z} f}(\theta)\right| \leq C \delta^{1+\nu},
$$

e portanto $n_{\mathrm{Z} f}(x)=N+n_{\mathrm{Z} f}(x)-N=N+\mathrm{O}\left(\delta^{1+\nu}\right)$. Então,

$$
\begin{aligned}
& \int_{0}^{t} n_{\mathrm{Z} f}(x) d x= \int_{0}^{t}\left(N+\mathrm{O}\left(\delta^{1+\nu}\right)\right) d x=N t+t \mathrm{O}\left(\delta^{1+\nu}\right) \\
& e^{\int_{0}^{t} n_{\mathrm{Z} f}(y) d y}=e^{\left(N t+t \mathrm{O}\left(\delta^{1+\nu}\right)\right)} \\
&=1+t\left(N+\mathrm{O}\left(\delta^{1+\nu}\right)\right)+\mathrm{O}\left(t^{2}\left(N+\mathrm{O}\left(\delta^{1+\nu}\right)\right)^{2}\right) \\
&=1+t\left(N+\mathrm{O}\left(\delta^{1+\nu}\right)\right)+t^{2} \mathrm{O}\left(\delta^{2}\right), \\
& \int_{0}^{x} e^{\int_{0}^{t} n_{\mathrm{Z} f}(y) d y} d t=\int_{0}^{x}\left(1+t\left(N+\mathrm{O}\left(\delta^{1+\nu}\right)\right)+t^{2} \mathrm{O}\left(\delta^{2}\right)\right) d t \\
&=x+N \frac{x^{2}}{2}+\mathrm{O}\left(\delta^{1+\nu}\right) \frac{x^{2}}{2}+\mathrm{O}\left(\delta^{2}\right) \frac{x^{3}}{3} \\
&=x\left(1+N \frac{x}{2}+\mathrm{O}\left(\delta^{1+\nu}\right)\right)
\end{aligned}
$$

e

$$
\int_{0}^{1} e^{\int_{0}^{t} n(y) d y} d t=1+\frac{N}{2}+\mathrm{O}\left(\delta^{1+\nu}\right)
$$


Portanto,

$$
\begin{aligned}
& \mathrm{Z} f(x)=\frac{\int_{0}^{x} e^{\int_{0}^{t} n_{\mathrm{Z} f}(y) d y} d t}{\int_{0}^{1} e^{\int_{0}^{t} n_{\mathrm{Z} f}(y) d y} d t}=\frac{x\left(1+N \frac{x}{2}+\mathrm{O}\left(\delta^{1+\nu}\right)\right)}{1+\frac{N}{2}+\mathrm{O}\left(\delta^{1+\nu}\right)} \\
& =x\left(1+N \frac{x}{2}+\mathrm{O}\left(\delta^{1+\nu}\right)\right)\left(1-\frac{N}{2}+\mathrm{O}\left(\delta^{1+\nu}\right)\right) \\
& =x\left(1-\frac{N}{2}+\frac{N}{2} x+\mathrm{O}\left(\delta^{1+\nu}\right)\right) \text {; } \\
& \operatorname{DZf}(x)=\frac{e^{\int_{0}^{x} n_{\mathrm{Z} f}(y) d y}}{\int_{0}^{1} e^{\int_{0}^{t} n_{\mathrm{Z} f}(y) d y} d t}=\frac{e^{\int_{0}^{x}\left(N+\mathrm{O}\left(\delta^{1+\nu}\right)\right) d y}}{1+\frac{N}{2}+\mathrm{O}\left(\delta^{1+\nu}\right)} \\
& =\frac{e^{\left(N x+x \mathrm{O}\left(\delta^{1+\nu}\right)\right)}}{1+\frac{N}{2}+\mathrm{O}\left(\delta^{1+\nu}\right)} \\
& =\left(1+N x+\mathrm{O}\left(\delta^{1+\nu}\right)\right)\left(1-\frac{N}{2}+\mathrm{O}\left(\delta^{1+\nu}\right)\right) \\
& =1+N x-\frac{N}{2}+\mathrm{O}\left(\delta^{1+\nu}\right) \text {; } \\
& D^{2} \mathrm{Z} f(x)=\frac{n_{\mathrm{Z} f}(x)}{\int_{0}^{1} e^{\int_{0}^{t} n_{\mathrm{Z} f}(y) d y} d t}=\frac{N+\mathrm{O}\left(\delta^{1+\nu}\right)}{1+\frac{N}{2}+\mathrm{O}\left(\delta^{1+\nu}\right)} \\
& =\left(N+\mathrm{O}\left(\delta^{1+\nu}\right)\right)\left(1-\frac{N}{2}+\mathrm{O}\left(\delta^{1+\nu}\right)\right) \\
& =N+\mathrm{O}\left(\delta^{1+\nu}\right) \text {. }
\end{aligned}
$$

Comparando as expressões de $\mathrm{Z} f, D \mathrm{Z} f, D^{2} \mathrm{Z} f$ com as de $\mathrm{Z} f_{N}, D \mathrm{Z} f_{N}, D^{2} \mathrm{Z} f_{N}$ obtidas no Lema 3.1.1 nós vemos que

$$
\mathrm{d}_{C^{2}}\left(\mathrm{Z} f, \mathrm{Z} f_{N}\right)=\mathrm{O}\left(\delta^{1+\nu}\right)
$$

Sejam $f_{i}:\left[a_{i}, b_{i}\right] \rightarrow\left[f\left(a_{i}\right), f\left(b_{i}\right)\right]$ difeomorfismos preservando orientação, de classe $C^{2+\nu}$. Por simplicidade, usaremos a seguinte notação:

$$
\mathrm{Z}_{\left[a_{i}, b_{i}\right]} f_{i}=\tilde{f}_{i}, M_{i}=M_{N_{f_{i}}}
$$




$$
\tilde{f}_{1}^{n}=\tilde{f}_{n} \circ \tilde{f}_{n-1} \circ \cdots \circ \tilde{f}_{1} \text { e } M_{1}^{n}=M_{n} \circ M_{n-1} \circ \cdots \circ M_{1} .
$$

Nosso próximo resultado dá uma estimativa entre composições de $\tilde{f}_{i}^{\prime} s$ e composições de $M_{i}^{\prime} s$.

Proposição 3.1.2. Sejam $f_{i}:\left[a_{i}, b_{i}\right] \rightarrow\left[f\left(a_{i}\right), f\left(b_{i}\right)\right]$ difeomorfismos preservando orientação, de classe $C^{2+\nu}$ em todos os intervalos $\left[a_{i}, b_{i}\right]$ e suponha que exista uma constante $C_{2}>0$ tal que $\sum_{i=1}^{n} \delta_{i} \leq C_{2}$, onde $\delta_{i}=b_{i}-a_{i}$. Então existe uma constante $C_{3}>0$ tal que

$$
\left|\tilde{f}_{1}^{n}-M_{1}^{n}\right|_{C^{2}} \leq C_{3} \cdot\left(\max _{1 \leq j \leq n} \delta_{j}\right)^{\nu} .
$$

Antes de provarmos a Proposição 3.1.2 precisaremos de alguns lemas.

Lema 3.1.2. Existe constante $C_{4}>0$ tal que

$$
e^{-C_{4}} \leq D \tilde{f}_{1}^{n}(x) \leq e^{C_{4}}
$$

para todo $x \in[0,1]$ e para todo $n \geq 0$.

Demonstração.

$$
\begin{aligned}
\ln \frac{D \tilde{f}_{1}^{n}(x)}{D \tilde{f}_{1}^{n}(y)} & =\ln \frac{D \tilde{f}_{n}\left(\tilde{f}_{1}^{n-1}(x)\right) \cdot D \tilde{f}_{n-1}\left(\tilde{f}_{1}^{n-2}(x)\right) \cdots D \tilde{f}_{1}(x)}{D \tilde{f}_{n}\left(\tilde{f}_{1}^{n-1}(y)\right) \cdot D \tilde{f}_{n-1}\left(\tilde{f}_{1}^{n-2}(y)\right) \cdots D \tilde{f}_{1}(y)} \\
& =\sum_{j=1}^{n} \ln D \tilde{f}_{j}\left(\tilde{f}_{1}^{j-1}(x)\right)-\ln D \tilde{f}_{j}\left(\tilde{f}_{1}^{j-1}(y)\right) \\
& =\sum_{j=1}^{n} \int_{\tilde{f}_{1}^{j-1}(y)}^{\tilde{f}_{1}^{j-1}(x)} \frac{D^{2} \tilde{f}_{j}(s)}{D \tilde{f}_{j}(s)} d s \\
& =\sum_{j=1}^{n} \frac{D^{2} \tilde{f}_{j}(z)}{D \tilde{f}_{j}(z)}\left|\tilde{f}_{1}^{j-1}(x)-\tilde{f}_{1}^{j-1}(y)\right|,
\end{aligned}
$$

para algum $z \in\left[\tilde{f}_{1}^{j-1}(y), \tilde{f}_{1}^{j-1}(x)\right]$.

Entretanto, pelo Lema 3.0.3 nós temos

$$
\ln \frac{D \tilde{f}_{1}^{n}(x)}{D \tilde{f}_{1}^{n}(y)} \leq \sum_{j=1}^{n}\left|\frac{D^{2} \tilde{f}_{j}(z)}{D \tilde{f}_{j}(z)}\right| \leq C_{1} \cdot \sum_{j=1}^{n} \delta_{j} \leq C_{1} C_{2}=C_{4} .
$$

Tomando $y \in[0,1]$ tal que $D \tilde{f}_{1}^{n}(y)=1$ temos o resultado.

Lema 3.1.3. Existe constante $C_{5}>0$ tal que

$$
\left|D^{2} \tilde{f}_{1}^{n}(x)\right| \leq C_{5},
$$

para todo $x \in[0,1]$ e para todo $n \geq 0$. 
Demonstração. Note que

$$
\begin{aligned}
\left|D^{2} \tilde{f}_{1}^{n}(x)\right| & =\left|\frac{D^{2} \tilde{f}_{1}^{n}(x)}{D \tilde{f}_{1}^{n}(x)}\right| \cdot\left|D \tilde{f}_{1}^{n}(x)\right| \\
& =\left|\sum_{j=1}^{n} \frac{D^{2} \tilde{f}_{j}\left(\tilde{f}_{1}^{j-1}(x)\right)}{D \tilde{f}_{j}\left(\tilde{f}_{1}^{j-1}(x)\right)} \cdot D \tilde{f}_{1}^{j-1}(x)\right| \cdot\left|D \tilde{f}_{1}^{n}(x)\right| \\
& \leq e^{2 C_{4}} \sum_{j=1}^{n}\left|\frac{D^{2} \tilde{f}_{j}\left(\tilde{f}_{1}^{j-1}(x)\right)}{D \tilde{f}_{j}\left(\tilde{f}_{1}^{j-1}(x)\right)}\right| \\
& \leq e^{2 C_{4}} \cdot C_{1} \cdot \sum_{i=1}^{n} \delta_{i} \\
& \leq e^{2 C_{4}} \cdot C_{1} \cdot C_{2}=C_{5} .
\end{aligned}
$$

Lema 3.1.4. Existem constantes $C_{6}, C_{7}>0$ tais que

$$
e^{-C_{6}} \leq\left|D M_{1}^{n}(x)\right| \leq e^{C_{6}} \text { e }\left|D^{2} M_{1}^{n}(x)\right| \leq C_{7},
$$

para todo $x \in[0,1]$ e para todo $n \geq 0$.

Demonstração. Análoga ao do Lema 3.1.2 e ao do Lema 3.1.3.

Demonstração. da Proposição 3.1.1:

Nós escrevemos

$$
\tilde{f}_{1}^{n}-M_{1}^{n}=\sum_{i=1}^{n} M_{i+1}^{n} \circ \tilde{f}_{1}^{i}-M_{i}^{n} \circ \tilde{f}_{1}^{i-1},
$$

onde $M_{n+1}^{n}=f_{1}^{0}=$ Id. Então

$$
\begin{aligned}
\left|\tilde{f}_{1}^{n}(x)-M_{1}^{n}(x)\right| & \leq \sum_{i=1}^{n}\left|M_{i+1}^{n}\left(\tilde{f}_{i} \circ \tilde{f}_{1}^{i-1}\right)(x)-M_{i+1}^{n}\left(M_{i} \circ \tilde{f}_{1}^{i-1}\right)(x)\right| \\
& \leq e^{C_{6}} \cdot \sum_{i=1}^{n}\left|\tilde{f}_{i} \circ \tilde{f}_{1}^{i-1}(x)-M_{i} \circ \tilde{f}_{1}^{i-1}(x)\right| \\
& \leq e^{C_{6}} \cdot C \cdot \sum_{i=1}^{n} \delta_{i}^{1+\nu} \\
& \leq e^{C_{6}} \cdot C \cdot\left(\max _{1 \leq i \leq n} \delta_{i}\right)^{\nu} \sum_{i} \delta_{i} \\
& \leq e^{C_{6}} \cdot C \cdot C_{2} \cdot\left(\max _{1 \leq i \leq n} \delta_{i}\right)^{\nu}
\end{aligned}
$$


onde $C>0$ é a constante dada pela Proposição 3.1.1.

$$
\begin{aligned}
\left|D \tilde{f}_{1}^{n}(x)-D M_{1}^{n}(x)\right|= & \mid \sum_{i=1}^{n}\left[D M_{i+1}^{n}\left(\tilde{f}_{i} \circ \tilde{f}_{1}^{i-1}(x)\right) \cdot D \tilde{f}_{i}\left(\tilde{f}_{1}^{i-1}(x)\right)\right. \\
& \left.-D M_{i+1}^{n}\left(M_{i} \circ \tilde{f}_{1}^{i-1}(x)\right) \cdot D M_{i}\left(\tilde{f}_{1}^{i-1}(x)\right)\right] D \tilde{f}_{1}^{i-1}(x) \mid \\
\leq & e^{C_{4}} \cdot \sum_{i=1}^{n} \mid D M_{i+1}^{n}\left(\tilde{f}_{i} \circ \tilde{f}_{1}^{i-1}(x)\right) \cdot D \tilde{f}_{i}\left(\tilde{f}_{1}^{i-1}(x)\right) \\
& -D M_{i+1}^{n}\left(M_{i} \circ \tilde{f}_{1}^{i-1}(x)\right) \cdot D M_{i}\left(\tilde{f}_{1}^{i-1}(x)\right) \mid .
\end{aligned}
$$

Adicionando e subtraindo o termo $D M_{i+1}^{n}\left(\tilde{f}_{i} \circ \tilde{f}_{1}^{i-1}(x)\right) \cdot D M_{i}\left(\tilde{f}_{1}^{i-1}(x)\right)$ na expressão acima, nós obtemos

$$
\begin{aligned}
\left|D \tilde{f}_{1}^{n}(x)-D M_{1}^{n}(x)\right| \leq & e^{C_{4}} \cdot e^{C_{6}} \cdot \sum_{i=1}^{n}\left|D \tilde{f}_{i}\left(\tilde{f}_{1}^{i-1}(x)\right)-D M_{i}\left(\tilde{f}_{1}^{i-1}(x)\right)\right| \\
& \quad+e^{C_{4}} \cdot e^{2 C_{6}} \cdot \sum_{i=1}^{n}\left|\tilde{f}_{i} \circ \tilde{f}_{1}^{i-1}(x)-M_{i} \circ \tilde{f}_{1}^{i-1}(x)\right| \\
\leq & e^{C_{4}} \cdot e^{2 C_{6}} \cdot C \cdot \sum_{i=1}^{n} \delta_{i}^{1+\nu} \\
\leq & e^{C_{4}} \cdot e^{2 C_{6}} \cdot C \cdot\left(\max _{1 \leq i \leq n} \delta_{i}\right)^{\nu} \cdot \sum_{i=1}^{n} \delta_{i} \\
\leq & e^{C_{4}} \cdot e^{2 C_{6}} \cdot C \cdot C_{2} \cdot\left(\max _{1 \leq i \leq n} \delta_{i}\right)^{\nu} .
\end{aligned}
$$

Agora, note que

$$
D^{2} \tilde{f}_{1}^{n}(x)-D^{2} M_{1}^{n}(x)=(\mathrm{I})+(\mathrm{II})+(\mathrm{III}),
$$

onde

$$
\begin{aligned}
(\mathrm{I})= & \sum_{i=1}^{n} D^{2} M_{i+1}^{n}\left(\tilde{f}_{i} \circ \tilde{f}_{1}^{i-1}(x)\right) \cdot\left(D \tilde{f}_{i}\left(\tilde{f}_{1}^{i-1}(x)\right)\right)^{2} \cdot\left(D \tilde{f}_{1}^{i-1}(x)\right)^{2} \\
& -\sum_{i=1}^{n} D^{2} M_{i+1}^{n}\left(M_{i} \circ \tilde{f}_{1}^{i-1}(x)\right) \cdot\left(D M_{i}\left(\tilde{f}_{1}^{i-1}(x)\right)\right)^{2} \cdot\left(D \tilde{f}_{1}^{i-1}(x)\right)^{2}, \\
(\mathrm{II})= & \sum_{i=1}^{n} D M_{i+1}^{n}\left(\tilde{f}_{i} \circ \tilde{f}_{1}^{i-1}(x)\right) \cdot D^{2} \tilde{f}_{i}\left(\tilde{f}_{1}^{i-1}(x)\right) \cdot\left(D \tilde{f}_{1}^{i-1}(x)\right)^{2} \\
& -\sum_{i=1}^{n} D M_{i+1}^{n}\left(M_{i} \circ \tilde{f}_{1}^{i-1}(x)\right) \cdot D^{2} M_{i}\left(\tilde{f}_{1}^{i-1}(x)\right) \cdot\left(D \tilde{f}_{1}^{i-1}(x)\right)^{2}
\end{aligned}
$$


e

$$
\begin{aligned}
(\mathrm{III})= & \sum_{i=1}^{n} D M_{i+1}^{n}\left(\tilde{f}_{i} \circ \tilde{f}_{1}^{i-1}(x)\right) \cdot D \tilde{f}_{i}\left(\tilde{f}_{1}^{i-1}(x)\right) \cdot D^{2} \tilde{f}_{1}^{i-1}(x) \\
& -\sum_{i=1}^{n} D M_{i+1}^{n}\left(M_{i} \circ \tilde{f}_{1}^{i-1}(x)\right) \cdot D M_{i}\left(\tilde{f}_{1}^{i-1}(x)\right) \cdot D^{2} \tilde{f}_{1}^{i-1}(x) .
\end{aligned}
$$

Para estimar (I) nós adicionaremos e subtrairemos o termo

$$
\sum_{i=1}^{n} D^{2} M_{i+1}^{n}\left(\tilde{f}_{i} \circ \tilde{f}_{1}^{i-1}(x)\right) \cdot\left(D M_{i}\left(\tilde{f}_{1}^{i-1}(x)\right)\right)^{2} \cdot\left(D \tilde{f}_{1}^{i-1}(x)\right)^{2}
$$

e usaremos o Lema 3.1.2 e o Lema 3.1.4.

Seja

$$
C_{8}=\sup _{1 \leq i \leq n} \sup _{x \in[0,1]}\left\{\left|D^{3} M_{i}(x)\right|\right\}
$$




$$
\begin{aligned}
|(\mathrm{I})| \leq & \sum_{i=1}^{n}\left\{\left[D^{2} M_{i+1}^{n}\left(\tilde{f}_{i} \circ \tilde{f}_{1}^{i-1}(x)\right) \cdot\left(D \tilde{f}_{i}\left(\tilde{f}_{1}^{i-1}(x)\right)\right)^{2}\right.\right. \\
& \left.\left.-D^{2} M_{i+1}^{n}\left(\tilde{f}_{i} \circ \tilde{f}_{1}^{i-1}(x)\right) \cdot\left(D M_{i}\left(\tilde{f}_{1}^{i-1}(x)\right)\right)^{2}\right]\left(D \tilde{f}_{1}^{i-1}(x)\right)^{2}\right\} \\
& +\sum_{i=1}^{n} D^{2} M_{i+1}^{n}\left(\tilde{f}_{i} \circ \tilde{f}_{1}^{i-1}(x)\right) \cdot\left(D M_{i}\left(\tilde{f}_{1}^{i-1}(x)\right)\right)^{2} \cdot\left(D \tilde{f}_{1}^{i-1}(x)\right)^{2} \\
& -\sum_{i=1}^{n} D^{2} M_{i+1}^{n}\left(M_{i} \circ \tilde{f}_{1}^{i-1}(x)\right) \cdot\left(D M_{i}\left(\tilde{f}_{1}^{i-1}(x)\right)\right)^{2} \cdot\left(D \tilde{f}_{1}^{i-1}(x)\right)^{2} \\
\leq & e^{2 C_{4}} \cdot C_{7} \cdot \sum_{i=1}^{n}\left[\left(D \tilde{f}_{i}\left(\tilde{f}_{1}^{i-1}(x)\right)\right)^{2}-\left(D M_{i}\left(\tilde{f}_{1}^{i-1}(x)\right)\right)^{2}\right] \\
& +e^{2 C_{4}+2 C_{6}} \cdot \sum_{i=1}^{n}\left|D^{2} M_{i+1}^{n}\left(\tilde{f}_{i} \circ \tilde{f}_{1}^{i-1}(x)\right)-D^{2} M_{i+1}^{n}\left(M_{i} \circ \tilde{f}_{1}^{i-1}(x)\right)\right| \\
\leq & e^{2 C_{4}} \cdot C_{7} \sum_{i=1}^{n}\left[\left|D \tilde{f}_{i}\left(\tilde{f}_{1}^{i-1}(x)\right)-D M_{i}\left(\tilde{f}_{1}^{i-1}(x)\right)\right| \cdot\left|D \tilde{f}_{i}\left(\tilde{f}_{1}^{i-1}(x)\right)+D M_{i}\left(\tilde{f}_{1}^{i-1}(x)\right)\right|\right] \\
& +e^{2 C_{4}+2 C_{6}} \cdot C_{8} \cdot \sum_{i=1}^{n}\left|\tilde{f}_{i} \circ \tilde{f}_{1}^{i-1}(x)-M_{i} \circ \tilde{f}_{1}^{i-1}(x)\right| \\
\leq & e^{2 C_{4}} \cdot C_{7} \cdot\left(e^{C_{4}}+e^{C_{6}}\right) \cdot \sum_{i=1}^{n}\left|D \tilde{f}_{i}\left(\tilde{f}_{1}^{i-1}(x)\right)-D M_{i}\left(\tilde{f}_{1}^{i-1}(x)\right)\right| \\
& +e^{2 C_{4}+2 C_{6}} \cdot C_{8} \cdot C \cdot \sum_{i=1}^{n} \delta_{i}^{1+\nu} \\
\leq & e^{2 C_{4}} \cdot C_{7} \cdot\left(e^{C_{4}}+C_{7} \cdot\left(e^{C_{6}}\right) \cdot C \cdot \sum_{i=1}^{n} \delta_{i}^{1+\nu}+e^{2 C_{4}+2 C_{6}} \cdot C_{8} \cdot C \cdot \sum_{i=1}^{n} \delta_{i}^{1+\nu} \cdot C_{2} \cdot\left(\max _{1 \leq i \leq n} \delta_{i}\right)^{\nu}+e^{2 C_{4}+2 C_{6}} \cdot C_{8} \cdot C \cdot C_{2} \cdot\left(\max _{1 \leq i \leq n} \delta_{i}\right)^{\nu}\right. \\
\leq & \left.\max _{9}, C_{10}\right\} \cdot\left(\max _{1 \leq i \leq n} \delta_{i}\right)^{\nu}, \\
& \\
& \\
& \\
&
\end{aligned}
$$

onde $C_{9}=e^{2 C_{4}} \cdot C_{7} \cdot\left(e^{C_{4}}+e^{C_{6}}\right) \cdot C \cdot C_{2}$ e $C_{10}=e^{2 C_{4}+2 C_{6}} \cdot C_{8} \cdot C \cdot C_{2}$.

Para estimar (II) adicionamos e subtraímos o termo

$$
\sum_{i=1}^{n} D M_{i+1}^{n}\left(M i \circ \tilde{f}_{1}^{i-1}(x)\right) \cdot D^{2} \tilde{f}_{i}\left(\tilde{f}_{1}^{i-1}(x)\right) \cdot\left(D \tilde{f}_{1}^{i-1}(x)\right)^{2}
$$

e usaremos o Lema 3.1.2 e o Lema 3.1.4. 


$$
\begin{aligned}
& |(\mathrm{II})| \leq \sum_{i=1}^{n} D M_{i+1}^{n}\left(\tilde{f}_{i} \circ \tilde{f}_{1}^{i-1}(x)\right) \cdot D^{2} \tilde{f}_{i}\left(\tilde{f}_{1}^{i-1}(x)\right) \cdot\left(D \tilde{f}_{1}^{i-1}(x)\right)^{2} \\
& -\sum_{i=1}^{n} D M_{i+1}^{n}\left(M i \circ \tilde{f}_{1}^{i-1}(x)\right) \cdot D^{2} \tilde{f}_{i}\left(\tilde{f}_{1}^{i-1}(x)\right) \cdot\left(D \tilde{f}_{1}^{i-1}(x)\right)^{2} \\
& +\sum_{i=1}^{n} D M_{i+1}^{n}\left(M i \circ \tilde{f}_{1}^{i-1}(x)\right) \cdot D^{2} \tilde{f}_{i}\left(\tilde{f}_{1}^{i-1}(x)\right) \cdot\left(D \tilde{f}_{1}^{i-1}(x)\right)^{2} \\
& -\sum_{i=1}^{n} D M_{i+1}^{n}\left(M_{i} \circ \tilde{f}_{1}^{i-1}(x)\right) \cdot D^{2} M_{i}\left(\tilde{f}_{1}^{i-1}(x)\right) \cdot\left(D \tilde{f}_{1}^{i-1}(x)\right)^{2} \\
& \leq e^{2 C_{4}} \cdot C_{5} \cdot C_{7} \sum_{i=1}^{n}\left|\tilde{f}_{i} \circ \tilde{f}_{1}^{i-1}(x)-M i \circ \tilde{f}_{1}^{i-1}(x)\right| \\
& +e^{2 C_{4}} \cdot e^{C_{6}} \cdot \sum_{i=1}^{n}\left|D^{2} \tilde{f}_{i}\left(\tilde{f}_{1}^{i-1}(x)\right)-D^{2} M_{i}\left(\tilde{f}_{1}^{i-1}(x)\right)\right| \\
& \leq e^{2 C_{4}} \cdot C_{5} \cdot C_{7} \cdot C \cdot C_{2} \cdot\left(\max _{1 \leq i \leq n} \delta_{i}\right)^{\nu}+e^{2 C_{4}} \cdot e^{C_{6}} \cdot C \cdot C_{2} \cdot\left(\max _{1 \leq i \leq n} \delta_{i}\right)^{\nu} \\
& \leq \max \left\{C_{11}, C_{12}\right\} \cdot\left(\max _{1 \leq i \leq n} \delta_{i}\right)^{\nu},
\end{aligned}
$$

onde $C_{11}=e^{2 C_{4}} \cdot C_{5} \cdot C_{7} \cdot C \cdot C_{2}$ e $C_{12}=e^{2 C_{4}} \cdot e^{C_{6}} \cdot C \cdot C_{2}$.

Adicionando e subtraindo o termo

$$
\sum_{i=1}^{n} D M_{i+1}^{n}\left(\tilde{f}_{i} \circ \tilde{f}_{1}^{i-1}(x)\right) \cdot D M_{i}\left(\tilde{f}_{1}^{i-1}(x)\right) \cdot D^{2} \tilde{f}_{1}^{i-1}(x)
$$

em (III) e novamente usando o Lema (3.1.2) e o Lema (3.1.2), obtemos

$$
\begin{aligned}
|(\mathrm{III})| \leq & \sum_{i=1}^{n} D M_{i+1}^{n}\left(\tilde{f}_{i} \circ \tilde{f}_{1}^{i-1}(x)\right) \cdot D \tilde{f}_{i}\left(\tilde{f}_{1}^{i-1}(x)\right) \cdot D^{2} \tilde{f}_{1}^{i-1}(x) \\
& -\sum_{i=1}^{n} D M_{i+1}^{n}\left(\tilde{f}_{i} \circ \tilde{f}_{1}^{i-1}(x)\right) \cdot D M_{i}\left(\tilde{f}_{1}^{i-1}(x)\right) \cdot D^{2} \tilde{f}_{1}^{i-1}(x) \\
& +\sum_{i=1}^{n} D M_{i+1}^{n}\left(\tilde{f}_{i} \circ \tilde{f}_{1}^{i-1}(x)\right) \cdot D M_{i}\left(\tilde{f}_{1}^{i-1}(x)\right) \cdot D^{2} \tilde{f}_{1}^{i-1}(x) \\
& -\sum_{i=1}^{n} D M_{i+1}^{n}\left(M_{i} \circ \tilde{f}_{1}^{i-1}(x)\right) \cdot D M_{i}\left(\tilde{f}_{1}^{i-1}(x)\right) \cdot D^{2} \tilde{f}_{1}^{i-1}(x) \\
\leq & \max \left\{C_{13}, C_{14}\right\} \cdot\left(\max _{1 \leq i \leq n} \delta_{i}\right)^{\nu},
\end{aligned}
$$

where $C_{13}=C \cdot C_{2} \cdot C_{5} \cdot e^{C_{6}}$ and $C_{14}=C \cdot C_{2} \cdot C_{5} \cdot C_{7} \cdot e^{C_{6}}$.

Tomando $C_{3}=\max \left\{C_{9}, C_{10}, C_{11}, C_{12}, C_{13}, C_{14}\right\}$ nós obtemos o resultado. 



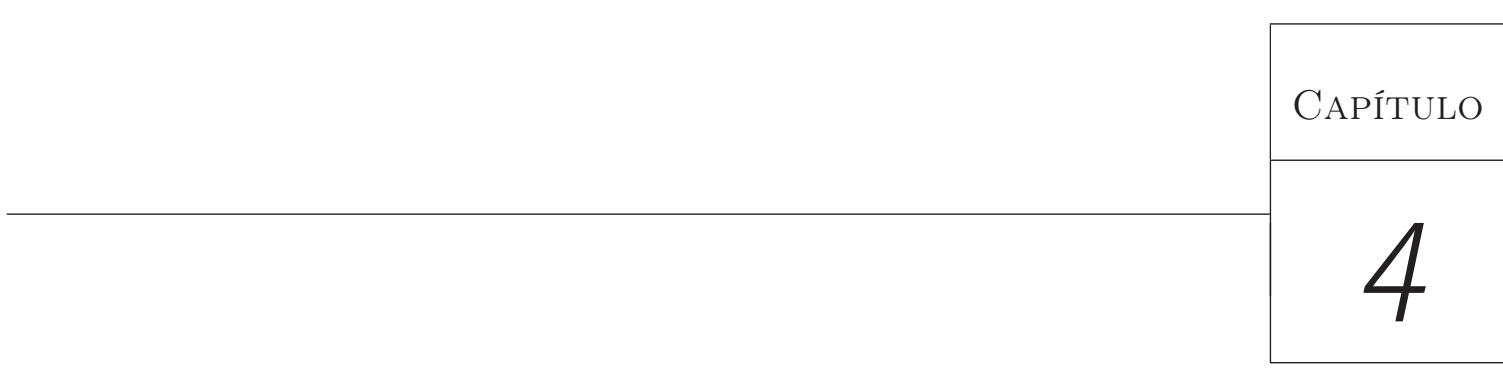

\section{Teorema B}

Neste capítulo nós provaremos que sob certas condições, a renormalização de $f$ converge, na topologia $C^{2}$, para o espaço formado pelas t.i.i. que restritas a cada subintervalo do domínio são Möbius. O resultado deste capítulo é inspirado no artigo [KV91].

Relembramos que $\mathcal{B}_{k}^{2+\nu}$ é o conjunto das transformações de intercâmbio de intervalos generalizadas $f$, tais que

(i) Para cada $\alpha \in \mathcal{A}$, temos que $\left.f\right|_{\overline{I_{\alpha}}}$ é um difeomorfismo de classe $C^{2+\nu}$;

(ii) $f$ tem combinatória $k$-limitada;

(iii) $f$ tem genus um;

(iv) $f$ não tem conexão.

Então temos o seguinte Teorema:

Teorema B. Seja $f \in \mathcal{B}_{k}^{2+\nu}$. Então existe constante $C=C(k)>0$ e $0<\lambda=\lambda(k)<1$, tal que

$$
\mathrm{d}_{C^{2}}\left(\mathcal{Z}_{I_{\alpha}^{n}}\left(\hat{R}^{n}(f)\right), M_{N_{\alpha}^{n}}\right) \leq C \lambda^{n}
$$

para todo $\alpha \in \mathcal{A}$, onde

$$
N_{\alpha}^{n}=\int_{I_{\alpha}^{n}} \frac{D^{2} \hat{R}^{n}(f)(x)}{D \hat{R}^{n}(f)(x)} d x
$$


Em particular,

$$
\mathrm{d}_{C^{2}}\left(\mathcal{Z}_{I_{\alpha}^{n}}\left(\hat{R}^{n}(f)\right), \mathcal{M}\right) \leq C \lambda^{n},
$$

onde $\mathcal{M}$ é o conjunto das transformações de Möbius $M:[0,1] \rightarrow[0,1]$, tal que $M(0)=0$ e $M(1)=1$.

Pela Proposição 3.1.2 temos que o zoom de $R^{n}(f)$ se aproxima de uma Möbius e cuja velocidade de aproximação é dada pelo máximo dos comprimentos dos intervalos $I_{\alpha}^{n}, \alpha \in \mathcal{A}$. Então para mostrarmos o Teorema $\mathrm{B}$, prescisamos mostrar que o comprimento destes intervalos vão para zero exponencialmente rápido, e que quaisquer dois subintervalos do domínio ou quaisquer dois subintervalos da imagem são comparáveis. Para mostrar isso nós precisaremos de uma versão mais forte do Teorema de Denjoy. Antes de enunciá-lo faremos uma pequena observação:

Observação 4.0.1. Seja $f \in \mathcal{B}_{k}$. É fácil ver que as únicas permutações que são invariantes pela renormalização, são permutações em que $f$ possui apenas uma descontinuidade. Como a combinatória $\left\{\pi^{i}, \varepsilon^{i}\right\}_{i \in \mathbb{N}}$ é $k$-limitada, temos que existe uma constante $C_{15}=C_{15}(k)>0$ tal que a aplicação $i \mapsto k_{i}$, onde $k_{i}$ é dado pelo Corolário 2.6.2. é uniformente limitada por $C_{15}$, ou seja, $\left|k_{i+1}-k_{i}\right| \leq C_{15}$ para todo $i \geq 0$.

Lema 4.0.5. Seja $f \in \mathcal{B}_{k}^{2}$. Existe constante $C_{16}=C_{16}(k, d)>0$ tal que

$$
\exp \left(-C_{16} V\right) \leq D R^{n}(x) \leq \exp \left(C_{16} V\right) \text { for all } x \in I^{n} .
$$

Demonstração. Pela Proposição 2.7.1, temos que

$$
\exp (-V) \leq D R^{n_{k_{i}}}(x) \leq \exp (V) \text { para todo } x \in I_{\alpha}^{n_{i}} .
$$

Seja $i \geq 0$ tal que $n_{k_{i}} \leq n<n_{k_{i+1}}$. Pela Observação 4.0.1 e pelo Corolário 2.6.1 temos que $\left|n_{k_{i+1}}-n_{k_{i}}\right| \leq d \cdot C_{15}$. Então $D R^{n}(f)=D R^{n_{k_{i}}+a_{n}}$, onde $a_{n} \leq C_{15}$. Tomando

$$
\begin{gathered}
C_{17}=\sup _{1 \leq i \leq C_{15}}\left\{D R^{i}(f)(x), x \in[0,1)\right\} \quad \mathrm{e} \\
C_{18}=\inf _{1 \leq i \leq C_{15}}\left\{D R^{i}(f)(x), x \in[0,1)\right\},
\end{gathered}
$$

temos

$$
C_{18} \cdot \exp (-V) \leq D R^{n}(f)=D R^{a_{n}}(f) \cdot D R^{n_{k_{i}}}(f) \leq C_{17} \cdot \exp (V) .
$$

Isto nos dá o resultado desejado.

Lema 4.0.6. Seja $f \in \mathcal{B}_{k}^{2}$. Existe constante $C_{19}>1$, tal que para todo $\alpha, \beta \in \mathcal{A}$ e para todo $n \geq 0$, os intervalos $I_{\alpha}^{n}$ e $I_{\beta}^{n}$ são $C_{19}$-comparáveis, ou seja,

$$
\frac{1}{C_{19}} \leq \frac{\left|I_{\alpha}^{n}\right|}{\left|I_{\beta}^{n}\right|} \leq C_{19} .
$$


Demonstração. Primeiro note que para todo $\alpha \in \mathcal{A}$, temos

- Se $\alpha$ não perde e não ganha na $n$-ésima renormalização então $\left|I_{\alpha}^{n}\right|=\left|I_{\alpha}^{n+1}\right|$;

- Se $\alpha$ ganha na $n$-ésima renormalização então $\left|I_{\alpha}^{n}\right|>\left|I_{\alpha}^{n+1}\right|$;

- Se $\alpha$ perde na $n$-ésima renormalização e $\varepsilon^{n}=1$ então $\left|I_{\alpha}^{n}\right|=\left|I_{\alpha}^{n+1}\right|$. Se $\varepsilon^{n}=0$ então $\left|f_{n}\left(I_{\alpha}^{n}\right)\right|=\left|f_{n+1}\left(I_{\alpha}^{n+1}\right)\right|$. Assim pelo Lema 4.0.5, $\left|I_{\alpha}^{n}\right| \geq \exp \left(-2 C_{16} V\right)\left|I_{\alpha}^{n+1}\right|$. Assim,

$$
\left|I_{\alpha}^{n}\right| \geq\left|I_{\alpha}^{n+1}\right|, \quad \text { para todo } \alpha \in \mathcal{A} \text {. }
$$

Suponhamos que o Lema é falso. Então existe $\alpha \in \mathcal{A}$ tal que o comprimento de $I_{\alpha}^{n}$ é suficientemente pequeno, digamos

$$
\left|I_{\alpha}^{n}\right| \leq \delta<\frac{1}{2 d \cdot \exp \left((k+2) C_{16} V\right)}
$$

Como a combinatória de $f$ é $k$-limitado, temos que para todo $\beta \in \mathcal{A}$ existem sequências $n \leq n_{0}<n_{1}<\ldots<n_{j+1} \leq n+k$ e $\alpha=\alpha_{0}, \alpha_{1}, \ldots, \alpha_{j+1}=\beta \in \mathcal{A}$ tal que $\alpha_{i}$ e $\alpha_{i+1}$ são as letras vencedoras e perdedoras, respectivamente da $n_{i}$-ésima renormalização. Logo, pelo Lema 4.0.5 temos

- Se $\varepsilon^{n_{0}}=0$ temos

$$
\left|I_{\alpha}^{n_{0}}\right|>\left|f_{n_{0}}\left(I_{\alpha_{1}}^{n_{0}}\right)\right| \geq \exp \left(-C_{16} V\right)\left|I_{\alpha_{1}}^{n_{0}}\right|
$$

- Se $\varepsilon^{n_{0}}=1$ temos

$$
\left|I_{\alpha_{1}}^{n_{0}}\right|<\left|f_{n_{0}}\left(I_{\alpha}^{n_{0}}\right)\right| \geq \exp \left(C_{16} V\right)\left|I_{\alpha}^{n_{0}}\right|
$$

Em ambos os casos obtemos

$$
\left|I_{\alpha}^{n_{0}}\right| \geq \exp \left(-C_{16} V\right)\left|I_{\alpha_{1}}^{n_{0}}\right|
$$

Por (4.1) e (4.3) temos

$$
\left|I_{\alpha}^{n}\right| \geq \exp \left(-C_{16} V\right)\left|I_{\alpha_{1}}^{n_{1}}\right| .
$$

Repetindo-se esse argumento $j+1$ vezes

$$
\left|I_{\alpha}^{n}\right| \geq \exp \left(-(j+1) C_{16} V\right)\left|I_{\alpha_{j+1}}^{n_{j+1}}\right| \geq \exp \left(-(k+2) C_{16} V\right)\left|I_{\beta}^{n+k}\right| .
$$

Da desigualdade acima obtemos

$$
\sum_{\beta \in \mathcal{A}}\left|I_{\beta}^{n+k}\right| \leq d \cdot \exp \left((k+2) C_{16} V\right)\left|I_{\alpha}^{n}\right|<\frac{1}{2}<1,
$$

o que é absurdo, pois

$$
\sum_{\beta \in \mathcal{A}}\left|I_{\beta}^{n+k}\right|=1
$$


Lema 4.0.7. Seja $f \in \mathcal{B}_{k}^{2}$. Existe constante $C_{20}>1$, tal que para todo $\alpha, \beta \in \mathcal{A}$ e para todo $n \geq 0$, os intervalos $f_{n}\left(I_{\alpha}^{n}\right)$ e $f_{n}\left(I_{\beta}^{n}\right)$ são $C_{20}$-comparáveis, ou seja,

$$
\frac{1}{C_{20}} \leq \frac{\left|f_{n}\left(I_{\alpha}^{n}\right)\right|}{\left|f_{n}\left(I_{\beta}^{n}\right)\right|} \leq C_{20} .
$$

Demonstração. Segue diretamente dos Lemas (4.0.5) e (4.0.6).

Lema 4.0.8. Let $f \in \mathcal{B}_{k}^{2}$ e $\alpha, \beta \in \mathcal{A}$ as letras vencedora e perdedora de $R^{n}(f)$, respectivamente. Então existe contantes $0<\lambda_{1}<\lambda_{2}<1$, tal que

$$
\lambda_{1}<\frac{\left|f^{(1-\varepsilon) q_{n}^{\beta}}\left(I_{\beta}^{n}\right)\right|}{\left|f^{\varepsilon q_{n}^{\alpha}}\left(I_{\alpha}^{n}\right)\right|}<\lambda_{2},
$$

onde $\varepsilon \in\{0,1\}$ é o tipo de $R^{n}(f)$.

Demonstração. Suponha que o lema é falso. Então ou $\left|f^{(1-\varepsilon) q_{n}^{\beta}}\left(I_{\beta}^{n}\right)\right|$ é muito pequeno, ou $\left|f^{(1-\varepsilon) q_{n}^{\beta}}\left(I_{\beta}^{n}\right)\right|-\left|f^{\varepsilon q_{n}^{\alpha}}\left(I_{\alpha}^{n}\right)\right|$ é muito pequeno. O primeiro caso não acontece pelos Lemas (4.0.6) e (4.0.7). Suponha então que o segundo caso acontece. Logo $\left|f^{\varepsilon q_{n}^{\alpha}}\left(I_{\alpha}^{n+1}\right)\right|=\left|f^{\varepsilon q_{n}^{\alpha}}\left(I_{\alpha}^{n}\right)\right|-\left|f^{(1-\varepsilon) q_{n}^{\beta}}\left(I_{\beta}^{n}\right)\right|$ é muito pequeno, o que contradiz novamente os Lemas (4.0.6) e (4.0.7).

Pela definição da renormalização de Rauzy-Veech, sabemos que

$$
[0,1)=\bigcup_{\alpha \in \mathcal{A}} \bigcup_{i=0}^{q_{n}^{\alpha}-1} f^{i}\left(I_{\alpha}^{n}\right),
$$

onde todas as uniões acima são disjuntas. Assim, os elementos $\left\{f^{i}\left(I_{\alpha}^{n}\right)\right\}$ formam uma partição de $[0,1)$, que denotaremos por $\mathcal{P}^{n}$. A norma de $\mathcal{P}^{n}$ é definida por

$$
\left|\mathcal{P}^{n}\right|=\max _{\substack{\alpha \in \mathcal{A} \\ 0 \leq i \leq q_{n}^{\alpha}-1}}\left\{\left|f^{i}\left(I_{\alpha}^{n}\right)\right|\right\}
$$

Nosso próximo resultado, nos diz que $\left|\mathcal{P}^{n}\right|$ tende a zero exponencialmente rápido.

Corolário 4.0.1. Seja $f \in \mathcal{B}_{k}^{2}$. Então existe constante $\lambda=\lambda\left(\lambda_{1}, \lambda_{2}\right)$ com $0<\lambda<1$ tal que

$$
\left|\mathcal{P}^{n+k}\right| \leq \lambda \cdot\left|\mathcal{P}^{n}\right| \text { para todo } n \geq 0
$$

Demonstração. Segue da definição de combinatória $k$-limitada e dos Lemas 4.0.5, 4.0.6, 4.0.7, 4.0.8.

A prova do Teorema B segue da Proposição 3.1.2 e do Corolário 4.0.1. 


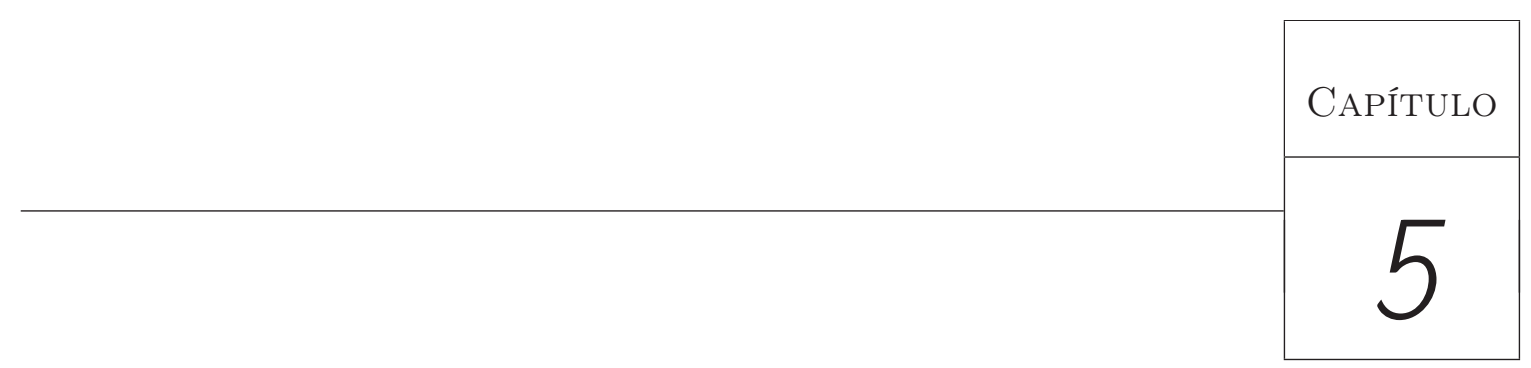

\section{Teoremas C e D}

Neste capítulo nós provaremos que se $f \in \mathcal{B}_{k}^{2+\nu}$ e $\int D^{2} f(s) / D f(s)=0$ então $R^{n} f$ converge na topologia $C^{2}$ para o espaço formado pelas t.i.i. afins, ou seja, o espaço das t.i.i. que restritas a cada subintervalo do domínio são afins. Este resultado é inspirado no artigo [KS87a].

Teorema C. Seja $f \in \mathcal{B}_{k}^{2+\nu}$. Então existem constantes $C=C(k)>0$ e $0<\lambda_{5}=$ $\lambda_{5}(k)<1$ tal que

$$
\left|N_{\alpha}^{n}-\frac{\sum_{i=0}^{q_{\alpha}^{n}-1}\left|f^{i}\left(I_{\alpha}^{n}\right)\right|}{|I|} \int_{0}^{1} \frac{D^{2} f(x)}{D f(x)} d x\right| \leq C \lambda_{5}^{\sqrt{n}}, \quad \text { para todo } \alpha \in \mathcal{A} .
$$

Em particular, se

$$
\int \frac{D^{2} f(x)}{D f(x)} d x=0
$$

então $\left|N_{\alpha}^{n}\right|<C \lambda_{5}^{\sqrt{n}}$ para todo $\alpha \in \mathcal{A}$.

O Teorema B junto com o Teorema C, nos dá o seguinte teorema:

Teorema D. Seja $f \in \mathcal{B}_{k}^{2+\nu}$ com

$$
\int \frac{D^{2} f(x)}{D f(x)} d x=0
$$


Então existem constantes $C=C(k)>0$ e $0<\lambda_{5}=\lambda_{5}(k)<1$ tal que

$$
\left|Z_{I_{\alpha}^{n}}\left(R^{n}(f)\right)-\mathrm{Id}\right|_{C^{2}} \leq C \cdot \lambda_{5}^{\sqrt{n}} \text { para todo } \alpha \in \mathcal{A} \text {. }
$$

Para mostrar o Teorema C, nós definimos uma dinâmica simbólica (inspirado por [KS87a]) cujos os cilindros dessa dinâmica são os elementos das partições definidas pelas renormalizações de $f$.

\subsection{Representação simbólica}

Para cada $n \in \mathbb{N}$ nós consideramos a seguinte partição do intervalo $[0,1)$ :

$$
\tilde{\mathcal{P}}^{n}=\left\{f^{i}\left(I_{\alpha}^{n}\right): \alpha \in \mathcal{A} \text { e } 1 \leq i \leq q_{n}^{\alpha}\right\}
$$

Considere o conjunto

$$
\mathcal{L}=\{(\alpha, \chi, n): \alpha \in \mathcal{A}, \chi \in\{0,1\}, n \in \mathbb{N}\},
$$

e $p_{2}, p_{3}$ a projeção de $\mathcal{L}$ em relação a segunda e terceira coordenada, respectivamente.

Nesta seção nós construiremos uma única representação simbólica para todos os pontos $x \in[0,1)$ no conjunto

$$
\Lambda=[0,1) \backslash \cup_{n} \cup_{J \in \tilde{\mathcal{P}}^{n}} \partial J .
$$

Seja $s: \Lambda \rightarrow \mathcal{L}^{\mathbb{N}}$, com $s(x)=\left(a_{n}\right)_{n \in \mathbb{N}}$, uma função definida da seguinte maneira: se $n=0$ então $x \in f\left(I_{\beta}^{0}\right)$ para algum $\beta \in \mathcal{A}$ e então definimos $a_{0}=(\beta, 0,0)$. Se $n>0$, seja

$$
k_{n-1}(x):=\min \left\{k \geq 0 \text { tal que } f^{k}(x) \in I^{n-1}\right\} .
$$

Então temos duas possibilidades mutualmente excludentes:

- ou $f^{k_{n-1}(x)}(x) \in I^{n}$, assim $x \in f_{n}\left(I_{\beta}^{n}\right)$ para algum $\beta \in \mathcal{A}$ e então definimos $a_{n}=(\beta, 0, n)$

- ou $f^{k_{n-1}(x)}(x) \in I^{n-1} \backslash I^{n}$, assim $f_{n-1}(x) \in f_{n}\left(I_{\beta}^{n}\right)$ para algum $\beta \in \mathcal{A}$ e então definimos $a_{n}=(\beta, 1, n)$.

Note que $p_{2}\left(a_{n}\right)=0$ se e somente se $k_{n}(x)=k_{n-1}(x)$. Defina $s_{n}(x)=a_{n}$.

Dado um subconjunto finito $S=\left\{n_{1}, n_{2}, \ldots, n_{k}\right\} \subset \mathbb{N}$. Uma sequência finita $a_{n_{1}}, \ldots, a_{n_{k}} \in \mathcal{L}$ indexada por $S$ será chamada palavra e denotada por

$$
\omega=a_{n_{k}} a_{n_{k-1}} \ldots a_{n_{1}}
$$




\subsubsection{Caracterização dos cilindros admissíveis e suas propriedades}

Para cada palavra $w$ nós podemos definir o cilindro

$$
[\omega]=\left[a_{n_{k}} a_{n_{k-1}} \ldots a_{n_{1}}\right]=\overline{\left\{x \in \Lambda \text { t.q. } \quad s_{n_{i}}(x)=a_{n_{i}}, 1 \leq i \leq k\right\}} .
$$

Se o cilindro $[\omega]$ é não vazio nós dizemos que a palavra $\omega$ é admissivel. Afirmamos que o conjunto formado pelo fecho dos intervalos em $\tilde{\mathcal{P}}^{n}$ é exatamente o conjunto dos cilindros da forma $\left[a_{n} a_{n-1} \ldots a_{0}\right]$, onde a palavra $a_{n} a_{n-1} \ldots a_{0}$ é admissível. De fato, quando $n=0$, temos que $\tilde{\mathcal{P}}^{0}=\left\{f\left(I_{\alpha}\right)\right\}_{\alpha \in \mathcal{A}}$. Então

$$
\overline{f\left(I_{\alpha}\right)}=[(\alpha, 0,0)] .
$$

Suponha por indução que todos os elementos da forma $f^{i}\left(I_{\alpha}^{n}\right)$ para todo $1 \leq i \leq q_{n}^{\alpha}$ e para todo $\alpha \in \mathcal{A}$ é um cilindro da forma $\left[a_{n} a_{n-1} \ldots a_{0}\right]$. Relembremos que as letras $\alpha^{n}(\varepsilon), \alpha^{n}(1-\varepsilon) \in \mathcal{A}$ são as letras vencedoras e perdedoras, respectivamente, de $f_{n}$ se a mesma, tem tipo $\varepsilon$. Então

- Se $\alpha \neq \alpha^{n}(1-\varepsilon)$ então

$$
\overline{f^{i}\left(I_{\alpha}^{n+1}\right)}=\left[a_{n+1} a_{n} \ldots a_{0}\right]
$$

para cada $1 \leq i \leq q_{n+1}^{\alpha}=q_{n}^{\alpha}$, onde $a_{n+1}=(\alpha, 0, n+1)$ e $\overline{f^{i}\left(I_{\alpha}^{n}\right)}=\left[a_{n} \ldots a_{0}\right]$;

- Se $\alpha=\alpha^{n}(1-\varepsilon)$ e $1 \leq i \leq q_{n}^{\alpha^{n}(\varepsilon)}$ nós temos

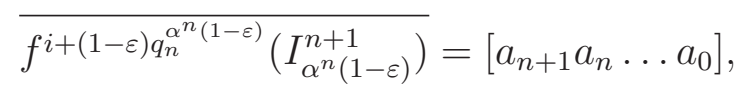

onde $a_{n+1}=\left(\alpha^{n}(1-\varepsilon), \varepsilon, n+1\right)$ e $\overline{f^{i}\left(I_{\alpha^{n}(\varepsilon)}^{n}\right)}=\left[a_{n} \ldots a_{0}\right]$;

- Se $\alpha=\alpha^{n}(1-\varepsilon)$ e $1 \leq j \leq q_{n}^{\alpha^{n}(1-\varepsilon)}$ nós temos

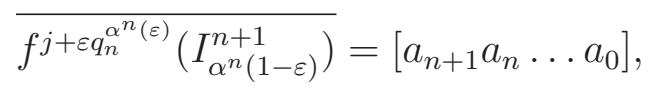

onde $a_{n+1}=\left(\alpha^{n}(1-\varepsilon), 1-\varepsilon, n+1\right)$ e $\overline{f^{j}\left(I_{\alpha^{n}(1-\varepsilon)}^{n}\right)}=\left[a_{n} \ldots a_{0}\right]$.

Observemos também que as funções $k_{1}, k_{2}, \ldots, k_{n}$ são constantes em $\left[a_{n} \ldots a_{0}\right]$, para qualquer palavra admissível $a_{n} a_{n-1} \ldots a_{0}$, e $f^{k_{n}}\left[a_{n} \ldots a_{0}\right]=f_{n}\left(I_{\alpha}^{n}\right)$, onde $\alpha \in \mathcal{A}$ é tal que $a_{n}=(\alpha, \chi, n)$.

A prova do Teorema 2 é baseado nas propriedades ergódicas da sequência de varáveis aleatórias $a_{n}$ com respeito a medida induzida pela medida de Lebesgue.

Se a palavra $a_{n} a_{n-1} \ldots a_{0}$ é admissível, nós podemos considerar a probabilidade condicional

$$
\ell\left(a_{n} \mid a_{n-1} \ldots a_{0}\right)=\frac{\ell\left(a_{n} a_{n-1} \ldots a_{0}\right)}{\ell\left(a_{n-1} \ldots a_{0}\right)}
$$


onde $\ell\left(a_{m} \ldots a_{0}\right)$ é a medida de Lebesgue do cilindro $\left[a_{m} \ldots a_{0}\right]$.

Lema 5.1.1. Sejam

$$
\begin{gathered}
\omega_{1}=a_{0}^{\prime} \ldots a_{n-1}^{\prime}, \\
\tilde{\omega}_{1}=a_{0}^{\prime \prime} \ldots a_{n-1}^{\prime \prime}, \\
\omega_{2}=a_{0}^{\prime} \ldots a_{n-1}^{\prime} a_{n}, \\
\tilde{\omega}_{2}=a_{0}^{\prime \prime} \ldots a_{n-1}^{\prime \prime}, a_{n}
\end{gathered}
$$

palavras admissiveis. Então existe um único $r \in \mathbb{Z}$ tal que $f^{r}$ é um difeomorfismo sobre $\left[\omega_{i}\right]$ e $f^{r}\left(\left[\omega_{i}\right]\right)=\left[\tilde{\omega}_{i}\right]$ para $i=1,2$.

Demonstração. A unicidade de $r$ segue do fato de que $f$ não tem pontos periódicos. De fato, se $f^{r_{1}}$ e $f^{r_{2}}$, com $r_{1}<r_{2}$ são difeomorfismos sobre $\left[\omega_{i}\right], f^{r_{i}}\left(\left[\omega_{i}\right]\right)=\left[\tilde{\omega}_{i}\right]$ para $i \in\{1,2\}$, então o ponto $\partial\left[\omega_{i}\right]$ é ponto fixo de $f^{r_{2}-r_{1}}$, o que é uma contradição.

Denote $a_{n-1}^{\prime}=\left(\alpha^{\prime}, \chi^{\prime}, n-1\right)$ e $a_{n-1}^{\prime \prime}=\left(\alpha^{\prime \prime}, \chi^{\prime \prime}, n-1\right)$. Pela definição da representação simbólica, temos que $\alpha^{\prime}=\alpha^{\prime \prime}=\alpha^{n-1}(1-\varepsilon)$ e se $\chi^{\prime}=\varepsilon$ então $\chi^{\prime \prime}=1-\varepsilon$. Assim

$$
\left[\omega_{1}\right]=\overline{f^{i_{0}}\left(I_{\alpha^{n-2}(1-\varepsilon)}^{n-1}\right)}=\overline{f^{i+(1-\varepsilon) q_{n-2}^{\alpha^{n-2}(1-\varepsilon)}\left(I_{\alpha^{n-2}(1-\varepsilon)}^{n-1}\right)},}
$$

para algum $1 \leq i \leq q_{n-3}^{\alpha^{n-3}(\varepsilon)} \mathrm{e}$

$$
\left[\tilde{\omega}_{1}\right]=\overline{f^{j 0}\left(I_{\alpha^{n-2}(1-\varepsilon)}^{n-1}\right)}=\overline{f^{j+\varepsilon q_{n-2}^{\alpha^{n-2}(\varepsilon)}}\left(I_{\alpha^{n-2}(1-\varepsilon)}^{n-1}\right)},
$$

para algum $1 \leq j \leq q_{n-3}^{\alpha^{n-3}(1-\varepsilon)}$. Supondo sem perda que $i_{0} \leq j_{0}$ e tomando $r=j_{0}-i_{0}$, temos que $f^{r}\left[\tilde{\omega}_{1}\right]=\left[\omega_{1}\right]$.

O fato de que $f^{r}\left[\tilde{\omega}_{2}\right]=\left[\omega_{2}\right]$ segue do fato de que $\left[\omega_{2}\right] \subset\left[\omega_{1}\right]$ e $\left[\tilde{\omega}_{2}\right] \subset\left[\tilde{\omega}_{1}\right]$.

Lema 5.1.2. Existem constantes $C_{19}>0$ and $0<\lambda_{3}=\lambda_{3}(\lambda)<1$ tal que

$$
e^{-C_{19} \lambda_{3}^{s}} \leq \frac{\ell\left(a_{n} \mid a_{n-1} \ldots a_{n-s} a_{n-s-1}^{\prime \prime} \ldots a_{0}^{\prime \prime}\right)}{\ell\left(a_{n} \mid a_{n-1} \ldots a_{n-s} a_{n-s-1}^{\prime} \ldots a_{0}^{\prime}\right)} \leq e^{C_{19} \lambda_{3}^{s}}
$$

dado que ambas as palavras são admissiveis.

Demonstração. Let $\overline{f^{i_{3}}\left(I_{\alpha}^{n-s}\right)}, \overline{f^{i_{2}}\left(I_{\beta}^{n}\right)}, \overline{f^{i_{1}}\left(I_{\gamma}^{n+1}\right)}$ os cilindros correspondentes as palavras

$$
a_{0}^{\prime} \ldots a_{n-s-1}^{\prime} a_{n-s} \ldots a_{n}, a_{0}^{\prime} \ldots a_{n-s-1}^{\prime} a_{n-s} \ldots a_{n-1}, a_{0}^{\prime} \ldots, a_{n-s-1}^{\prime}
$$


respectivamente. Pelo Lema (5.1.1) existe $j$ com $i_{3}+j \leq q_{n-s}^{\alpha}$, tal que $\overline{f^{i_{3}+j}\left(I_{\alpha}^{n-s}\right)}$, $\overline{f^{i_{2}+j}\left(I_{\beta}^{n}\right)}, \overline{f^{i_{1}+j}\left(I_{\gamma}^{n+1}\right)}$ são os cilindros correspondentes as palavras

$$
\left.\left.a_{0}^{\prime \prime} \ldots a_{n-s-1}^{\prime \prime} a_{n-s} \ldots a_{n}, a_{0}^{\prime \prime} \ldots a_{n-s-1}^{\prime \prime} a_{n-s} \ldots a_{n-1}\right) \text { e } a_{0}^{\prime \prime} \ldots a_{n-s-1}^{\prime \prime}\right)
$$

respectivamente. Defina para todo $1 \leq k \leq j$, o número

$$
\rho_{k}:=\frac{\left|f^{i_{2}}\left(I_{\beta}^{n+1}\right)\right| \cdot\left|f^{i_{1}+k}\left(I_{\gamma}^{n}\right)\right|}{\left|f^{i_{1}}\left(I_{\gamma}^{n}\right)\right| \cdot\left|f^{i_{2}+k}\left(I_{\beta}^{n+1}\right)\right|} .
$$

Assim,

$$
\begin{aligned}
\rho_{k+1} & :=\frac{\int_{f^{i_{1}+k}\left(I_{\gamma}^{n}\right)} D f(s) d s}{\int_{f^{i_{2}+k}\left(I_{\beta}^{n+1}\right)} D f(s) d s} \cdot \frac{\left|f^{i_{2}}\left(I_{\beta}^{n+1}\right)\right|}{\left|f^{i_{1}+k}\left(I_{\gamma}^{n}\right)\right|} \\
& =\frac{D f\left(s_{i_{1}+k}\right)\left|f^{i_{1}+k}\left(I_{\gamma}^{n}\right)\right|}{D f\left(s_{i_{2}+k}\right)\left|f^{i_{2}+k}\left(I_{\beta}^{n+1}\right)\right|} \cdot \frac{\left|f^{i_{2}}\left(I_{\beta}^{n+1}\right)\right|}{\left|f^{i_{1}+k}\left(I_{\gamma}^{n}\right)\right|} \\
& =\frac{D f\left(s_{i_{1}+k}\right)}{D f\left(s_{i_{2}+k}\right)} \cdot \rho_{k},
\end{aligned}
$$

onde $s_{i_{1}+k} \in f^{i_{1}+k}\left(I_{\gamma}^{n}\right)$ e $s_{i_{2}+k} \in f^{i_{2}+k}\left(I_{\beta}^{n+1}\right)$. Agora note que

$$
\begin{aligned}
\left|\ln \left(\frac{D f\left(s_{i_{1}+k}\right)}{D f\left(s_{i_{2}+k}\right)}\right)\right| & =\left|\ln D f\left(s_{i_{1}+k}\right)-\ln D f\left(s_{i_{2}+k}\right)\right| \leq C_{1} \cdot\left|s_{i_{1}+k}-s_{i_{2}+k}\right| \\
& \leq C_{1} \cdot\left|f^{i_{1}+k}\left(I_{\gamma}^{n}\right)\right|
\end{aligned}
$$

e portanto,

$$
\exp \left\{-C_{1} \cdot\left|f^{i_{1}+k}\left(I_{\gamma}^{n}\right)\right|\right\} \leq \frac{D f\left(s_{i_{1}+k}\right)}{D f\left(s_{i_{2}+k}\right)} \leq \exp \left\{C_{1} \cdot\left|f^{i_{1}+k}\left(I_{\gamma}^{n}\right)\right|\right\}
$$

Como $\rho_{j}=\prod_{t=0}^{j-1} \frac{D f\left(s_{i_{1}+t}\right)}{D f\left(s_{i_{2}+t}\right)}$, temos então por $(5.1)$

$$
\exp \left\{-C_{1} \sum_{t=0}^{j-1}\left|f^{i_{1}+t}\left(I_{\gamma}^{n}\right)\right|\right\} \leq \rho_{j} \leq \exp \left\{C_{1} \sum_{t=0}^{j-1}\left|f^{i_{1}+t}\left(I_{\gamma}^{n}\right)\right|\right\} .
$$

Entretanto, pela Proposição (4.0.1),

$$
\sum_{t=0}^{j-1}\left|f^{i_{1}+t}\left(I_{\gamma}^{n}\right)\right|=\sum_{t=0}^{j-1}\left|f^{i_{3}+t}\left(I_{\alpha}^{n-s}\right)\right| \cdot \frac{\left|f^{i_{1}+t}\left(I_{\gamma}^{n}\right)\right|}{\left|f^{i_{3}+t}\left(I_{\alpha}^{n-s}\right)\right|} \leq C_{20} \cdot \lambda_{3}^{s},
$$

onde $C_{20}=C_{20}\left(C_{17}, C_{18}, \lambda\right)>0$ é uma constante. Tomando $C_{19}=C_{1} \cdot C_{20}$, obtemos o resultado. 
O Lema (5.1.2) mostra que a sequência de variáveis aleatórias $\left\{a_{n}\right\}$ pode ser suficiente aproximada por cadeia de Markov.

Lema 5.1.3. Existe constante $C_{21}=C_{21}\left(C_{19}, \lambda\right)>0$ tal que para todo $n, m$,

$$
e^{-C_{21}} \leq \frac{\ell\left(a_{n+m}, \ldots, a_{n} \mid a_{n-1}^{\prime}, \ldots, a_{0}^{\prime}\right)}{\ell\left(a_{n+m}, \ldots, a_{n} \mid a_{n-1}^{\prime \prime}, \ldots, a_{0}^{\prime \prime}\right)} \leq e^{C_{21}},
$$

dado que ambas as palavras $\left(a_{0}^{\prime}, \ldots, a_{n-1}^{\prime}, a_{n}, \ldots, a_{n+m}\right)$ and $\left(a_{0}^{\prime \prime}, \ldots, a_{n-1}^{\prime \prime}, a_{n}, \ldots, a_{n+m}\right)$ são admissiveis.

Demonstração. Primeiro note que

$$
\begin{aligned}
& l\left(a_{n+m}, \ldots, a_{n} \mid a_{n-1}^{\prime}, \ldots, a_{0}^{\prime}\right)=\prod_{i=0}^{m} l\left(a_{n+i} \mid a_{n+i-1}, \ldots, a_{n-1}^{\prime}, \ldots, a_{0}^{\prime}\right), \\
& l\left(a_{n+m}, \ldots, a_{n} \mid a_{n-1}^{\prime \prime}, \ldots, a_{0}^{\prime \prime}\right)=\prod_{i=0}^{m} l\left(a_{n+i} \mid a_{n+i-1}, \ldots, a_{n-1}^{\prime \prime}, \ldots, a_{0}^{\prime \prime}\right) .
\end{aligned}
$$

Pelo Lema (5.1.2) temos que

$$
\frac{l\left(a_{n+i} \mid a_{n+i-1}, \ldots, a_{n-1}^{\prime}, \ldots, a_{0}^{\prime}\right)}{l\left(a_{n+i} \mid a_{n+i-1}, \ldots, a_{n-1}^{\prime \prime}, \ldots, a_{0}^{\prime \prime}\right)} \leq e^{C_{19} \cdot \lambda_{3}^{i}} .
$$

Logo,

$$
\begin{aligned}
\frac{l\left(a_{n+m}, \ldots, a_{n} \mid a_{n-1}^{\prime}, \ldots, a_{0}^{\prime}\right)}{l\left(a_{n+m}, \ldots, a_{n} \mid a_{n-1}^{\prime \prime}, \ldots, a_{0}^{\prime \prime}\right)} & =\prod_{i=0}^{m} \frac{l\left(a_{n+i} \mid a_{n+i-1}, \ldots, a_{n-1}^{\prime}, \ldots, a_{0}^{\prime}\right)}{l\left(a_{n+i} \mid a_{n+i-1}, \ldots, a_{n-1}^{\prime \prime}, \ldots, a_{0}^{\prime \prime}\right)} \\
& \leq \prod_{i=0}^{m} e^{C_{19} \cdot \lambda_{1}^{i}} \\
& =e^{C_{19} \sum_{i=0}^{m} \lambda_{1}^{i}} \\
& \leq e^{C_{21}}
\end{aligned}
$$

A outra desigualdade é obtida de forma similar.

Lema 5.1.4. Seja $a_{0} \ldots a_{n}$ and $a_{n}^{\prime} a_{n+1}^{\prime} \ldots a_{n+m}^{\prime}$ duas palavras admissíveis tal que $a_{n}=(\alpha, \chi, n), a_{n}^{\prime}=\left(\alpha, \chi^{\prime}, n\right)$. Então $a_{0} \ldots a_{n} a_{n+1}^{\prime} \ldots a_{n+m}^{\prime}$ é admissivel.

Demonstração. Se $a_{n}^{\prime} a_{n+1}^{\prime} \ldots a_{n+m}^{\prime}$ é admissível, então existe uma palavra admissível da forma $a_{0}^{\prime} \ldots a_{n}^{\prime} a_{n+1}^{\prime} \ldots a_{n+m}^{\prime}$. Note que as funções $k_{1}(x)=k_{1}^{\prime}, k_{2}(x)=k_{2}^{\prime}, \ldots$, $k_{n+m}(x)=k_{n+m}^{\prime}$ são constantes em $\left[a_{0}^{\prime} \ldots a_{n}^{\prime} a_{n+1}^{\prime} \ldots a_{n+m}^{\prime}\right] \mathrm{e}$

$$
f^{k_{n}^{\prime}}\left[a_{0}^{\prime} \ldots a_{n}^{\prime} a_{n+1}^{\prime} \ldots a_{n+m}^{\prime}\right] \subset f_{n}\left(I_{\alpha}^{n}\right), \text { pois } a_{n}^{\prime}=\left(\alpha, \chi^{\prime}, n\right) .
$$


As funções $k_{1}(x)=k_{1}, k_{2}(x)=k_{2}, \ldots, k_{n}(x)=k_{n}$ são constantes em $\left[a_{0} \ldots a_{n}\right] \mathrm{e}$

$$
f^{k_{n}}\left[a_{0} \ldots a_{n}\right]=f_{n}\left(I_{\alpha}^{n}\right), \text { pois } a_{n}=(\alpha, \chi, n) .
$$

Afirmamos que cada $x$ no conjunto não vazio

$$
J=f^{-k_{n}} f^{k_{n}^{\prime}}\left[a_{0}^{\prime} \ldots a_{n}^{\prime} a_{n+1}^{\prime} \ldots a_{n+m}^{\prime}\right] \cap \Lambda \subset\left[a_{0} \ldots a_{n}\right],
$$

pertence ao cilindro $\left[a_{0} \ldots a_{n} a_{n+1}^{\prime} \ldots a_{n+m}^{\prime}\right]$. De fato, desde que $x \in\left[a_{0} \ldots a_{n}\right]$ nós temos que $s_{i}(x)=a_{i}, k_{i}(x)=k_{i}$, para todo $0 \leq i \leq n$. Note que $f^{k_{n}}(x)=f^{k_{n}^{\prime}}(y)$, para algum $y \in\left[a_{0}^{\prime} \ldots a_{n}^{\prime} a_{n+1}^{\prime} \ldots a_{n+m}^{\prime}\right] \cap \Lambda$. Então

$$
f^{k_{i}^{\prime}}(y)=f^{k_{i}^{\prime}-k_{n}^{\prime}+k_{n}}(x) \in I^{i}, \text { para todo } n \leq i \leq n+m
$$

Assim, para todo $0 \leq j<k_{i}^{\prime}-k_{n}^{\prime}+k_{n}$ e para todo $n \leq i \leq n+m$, temos

$$
f^{j}(x)=f^{j-k_{n}+k_{n}^{\prime}}(y) \notin I^{i},
$$

pois $j-k_{n}+k_{n}^{\prime}<k_{i}^{\prime}$. Portanto

$$
k_{i}(x)=k_{i}(y)-k_{n}^{\prime}+k_{n}=k_{i}^{\prime}-k_{n}^{\prime}+k_{n} .
$$

Por (5.2) e (5.3), temos que $s_{i}(x)=a_{i}^{\prime}$ for $n<i \leq n+m$.

Lema 5.1.5. Seja $\alpha, \beta \in \mathcal{A}$. Para cada $n \geq 0$ existe uma palavra admissivel $a_{n} \ldots a_{n+k}$ com $a_{n}=(\alpha, \chi, n)$ e $a_{n+k}=\left(\beta, \chi^{\prime}, n+k\right)$.

Demonstração. Como a combinatória é $k$-limitada, sabemos que existem sequências $n \leq n_{0}<n_{2}<\ldots<n_{s} \leq n+k$ e $\alpha=\alpha_{0}, \alpha_{1}, \ldots, \alpha_{s}=\beta$ tal que $\alpha_{i}$ e $\alpha_{i+1}$ são as letras vencedoras e perdedoras para a $n_{i}$-ésima renormalização. Assim pela definição da dinâmica simbólica, sabemos que

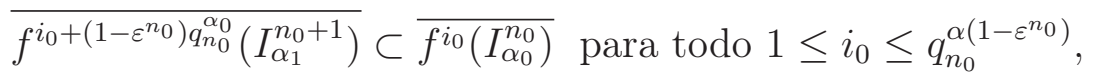

ou seja,

$$
\overline{f^{i_{1}}\left(I_{\alpha_{1}}^{n_{0}+1}\right)} \subset \overline{f^{i_{0}}\left(I_{\alpha}^{n_{0}}\right)}
$$

onde $i_{1}=i_{1}\left(i_{0}, \varepsilon^{n_{0}}\right)=i_{0}+\left(1-\varepsilon^{n_{0}}\right) q_{n_{0}}^{\alpha}$. Analogamente,

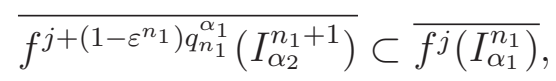

para todo $1 \leq j \leq q_{n_{1}}^{\alpha\left(1-\varepsilon^{n_{1}}\right)}$. 
Novamente pela definição da dinâmica simbólica e pelo Lema 5.1.4, existem $j$ e $i_{1}$ tal que

$$
\overline{f^{j}\left(I_{\alpha_{1}}^{n_{1}}\right)} \subset \overline{f^{i_{1}}\left(I_{\alpha_{1}}^{n_{0}+1}\right)} \subset \overline{f^{i_{0}}\left(I_{\alpha}^{n_{0}}\right)}
$$

Aplicado este processo $s-1$ vezes, obtemos o resultado.

Lema 5.1.6. Existe constante $C_{22}>0$ tal que para todo $n, m$, e qualquer palavras admissiveis $\left(a_{0}^{\prime}, \ldots, a_{n-k}^{\prime}\right),\left(a_{0}^{\prime \prime}, \ldots, a_{n-k}^{\prime \prime}\right),\left(a_{n}, \ldots, a_{n+m}\right)$ :

$$
e^{-C_{22}} \leq \frac{\ell\left(a_{n+m}, \ldots, a_{n} \mid a_{n-k}^{\prime}, \ldots, a_{0}^{\prime}\right)}{\ell\left(a_{n+m}, \ldots, a_{n} \mid a_{n-k}^{\prime \prime}, \ldots, a_{0}^{\prime \prime}\right)} \leq e^{C_{22}} .
$$

Demonstração. Segue do Lema 5.1.2 e do Lema 5.1.3.

Proposição 5.1.1. Existem constantes $C_{23}>0$ e $0<\lambda_{4}<1$ tal que

$$
\left|\ell\left(a_{n} \mid a_{n-r}, \ldots, a_{0}\right)-\ell\left(a_{n}=0\right)\right| \leq C_{23} \cdot \lambda_{4}^{\sqrt{r}},
$$

onde $r=\left[\frac{n}{2}\right]$.

Demonstração. A prova é baseada nos métodos da teoria de cadeias de Markov e é uma consequência do Teorema Ergódico para cadeias de Markov . Esta técnica é bem conhecida e sua prova pode ser encontrada em [Sin94a]. Assim nós descreveremos aqui os principais passos.

Fixe um inteiro $m, m \sim \sqrt{\frac{n}{2}}$ e introduza uma nova medida de probabilidade sobre as palavras

$$
\begin{aligned}
\tilde{a}= & \left(a_{n}, a_{n-1}, \ldots, a_{n-m+k}, a_{n-m}, \ldots, a_{n-2 m+k},\right. \\
& \left.a_{n-2 m}, \ldots, a_{n-3 m+k}, \ldots, a_{n-(i-1) m}, \ldots, a_{n-i m+k}, a_{n-i m}, \ldots, a_{0}\right)
\end{aligned}
$$

pela fórmula

$$
\begin{aligned}
\ell^{\prime}(\tilde{a})= & \ell\left(a_{0}, \ldots, a_{n-i m}\right) \ell\left(a_{n-(i-1) m}, \ldots, a_{n-i m+3} \mid a_{n-i m}, \ldots, a_{0}\right) \\
& \prod_{s=0}^{i-2} \ell\left(a_{n-s m}, \ldots, a_{n-(s+1) m+k} \mid a_{n-(s+1) m}, \ldots, a_{n-(s+2) m+k}\right),
\end{aligned}
$$

onde $i \sim \sqrt{\frac{n}{2}}$. Esta é uma medida de uma cadeia de Markov com memória $m$. Segue facilmente do Lema 5.1.2 que a medida $\ell^{\prime}$ é equivalente a medida $\ell$, isto é

$$
\exp \left(-C_{19} \cdot \lambda_{3}^{m} \cdot i\right) \leq \frac{\ell^{\prime}(\tilde{a})}{\ell(\tilde{a})} \leq \exp \left(C_{19} \cdot \lambda_{3}^{m} \cdot i\right)
$$

Considere o operador transição de Markov correspondente a $\ell^{\prime}$, para transição de $m$ passos. Assim pelo Lema 5.1.6 este é uma contração com coeficiente uniformemente 
menor do que 1. Então o Teorema Ergódico para cadeias de Markov mostra que a probabilidade condicional $\ell\left(a_{n} \mid a_{n-i m}, \ldots, a_{0}\right)$ para diferentes $a_{n-i m}, \ldots, a_{0}$ é assintoticamente um evento indepedente. Isto nos dá o resultado desejado.

\subsection{Prova dos Teoremas C e D.}

Proposição 5.2.1. Para todo $\alpha \in \mathcal{A}$ existe constante $0<\lambda_{5}=\lambda_{5}(k, \lambda)<1$ tal que

$$
\int_{I_{\alpha}^{n}} \frac{D^{2} f_{n}(s)}{D f_{n}(s)} d s=\ell((\alpha, 0, n)) \cdot \int_{I} \frac{D^{2} f(s)}{D f(s)} d s+O\left(\lambda_{5}^{\sqrt{\frac{n}{2}}}\right) .
$$

Demonstração. Seja $r=\left[\frac{n}{2}\right]$, o maior inteiro menor ou igual a $\frac{n}{2}$ e $\alpha \in \mathcal{A}$.

$$
\begin{aligned}
\int_{I_{\alpha}^{n}} \frac{D^{2} f_{n}(s)}{D f_{n}(s)} d s & =\sum_{i=1}^{q_{n}^{\alpha}} \int_{f^{i}\left(I_{\alpha}^{n}\right)} \frac{D^{2} f(s)}{D f(s)} d s \\
& =\sum_{\beta \in \mathcal{A}} \sum_{j=1}^{q_{r}^{\beta}} \sum_{i: f^{i}\left(I_{\alpha}^{n}\right) \subset f^{j}\left(I_{\beta}^{r}\right)} \int_{f^{i}\left(I_{\alpha}^{n}\right)} \frac{D^{2} f(s)}{D f(s)} d s \\
& \left.=\sum_{\beta \in \mathcal{A}} \sum_{j=1}^{q_{r}^{\beta}} \sum_{i: f^{i}\left(I_{\alpha}^{n}\right) \subset f^{j}\left(I_{\beta}^{r}\right)} \frac{D^{2} f\left(x_{j}\right)}{D f\left(x_{j}\right)}-\frac{D^{2} f\left(y_{j}^{\beta}\right)}{D f\left(y_{j}^{\beta}\right)}\right) \cdot\left|f^{i}\left(I_{\alpha}^{n}\right)\right| \\
& +\sum_{\beta \in \mathcal{A}} \sum_{j=1}^{q_{r}^{\beta}} \sum_{i: f^{i}\left(I_{\alpha}^{n}\right) \subset f^{j}\left(I_{\beta}^{r}\right)} \frac{D^{2} f\left(y_{j}^{\beta}\right)}{D f\left(y_{j}^{\beta}\right)} \cdot\left|f^{i}\left(I_{\alpha}^{n}\right)\right| \\
& =(\mathrm{I})+(\mathrm{II}),
\end{aligned}
$$

onde, $y_{j}^{\beta} \in f^{j}\left(I_{\beta}^{r}\right)$ e $x_{i} \in f^{i}\left(I_{\alpha}^{n}\right)$.

Agora estimaremos (I) : 


$$
\begin{aligned}
|(\mathrm{I})| & \leq \sum_{\beta \in \mathcal{A}} \sum_{j=1}^{q_{r}^{\beta}} \sum_{i: f^{i}\left(I_{\alpha}^{n}\right) \subset f^{j}\left(I_{\beta}^{r}\right)} C_{0} \cdot\left|x_{i}-y_{j}^{\beta}\right|^{\nu} \cdot\left|f^{i}\left(I_{\alpha}^{n}\right)\right| \\
& \leq C_{0} \cdot \sum_{\beta \in \mathcal{A}} \sum_{j=1}^{q_{r}^{\beta}} \sum_{i: f^{i}\left(I_{\alpha}^{n}\right) \subset f^{j}\left(I_{\beta}^{r}\right)}\left|f^{j}\left(I_{\beta}^{r}\right)\right|^{\nu} \cdot\left|f^{i}\left(I_{\alpha}^{n}\right)\right| \\
& \leq C_{0} \cdot \sum_{\beta \in \mathcal{A}} \sum_{j=1}^{q_{r}^{\beta}}\left|f^{j}\left(I_{\beta}^{r}\right)\right|^{\nu} \sum_{i: f^{i}\left(I_{\alpha}^{n}\right) \subset f^{j}\left(I_{\beta}^{r}\right)}\left|f^{i}\left(I_{\alpha}^{n}\right)\right| \\
& \leq C_{0} \cdot \sum_{\beta \in \mathcal{A}} \sum_{j=1}^{q_{r}^{\beta}}\left|f^{j}\left(I_{\beta}^{r}\right)\right|^{1+\nu} \\
& \leq C_{0} \cdot \sum_{\beta \in \mathcal{A}}\left(\max _{0 \leq j \leq q_{\beta}^{r}}\left|f^{j}\left(I_{\beta}^{r}\right)\right|\right)^{\nu} \cdot \sum_{j=1}^{q_{r}^{\beta}}\left|f^{j}\left(I_{\beta}^{r}\right)\right| \\
& \leq C_{0} \cdot \lambda^{\frac{n}{2} \nu} .
\end{aligned}
$$

Denote por $\omega_{j, \beta}$ a palavra adimissível tal que $\left[\omega_{j, \beta}\right]=\overline{f^{j}\left(I_{\beta}^{r}\right)}$. Assim

$$
\begin{aligned}
|(\mathrm{II})|= & \sum_{\beta \in \mathcal{A}} \sum_{j=1}^{q_{r}^{\beta}} \sum_{i: f^{i}\left(I_{\alpha}^{n}\right) \subset f^{j}\left(I_{\beta}^{r}\right)} \frac{D^{2} f\left(y_{j}^{\beta}\right)}{D f\left(y_{j}^{\beta}\right)} \cdot\left|f^{j}\left(I_{\beta}^{r}\right)\right| \cdot \frac{\left|f^{i}\left(I_{\alpha}^{n}\right)\right|}{\left|f^{j}\left(I_{\beta}^{r}\right)\right|} \\
= & \sum_{\beta \in \mathcal{A}} \sum_{j=1}^{q_{r}^{\beta}} \frac{D^{2} f\left(y_{j}^{\beta}\right)}{D f\left(y_{j}^{\beta}\right)} \cdot\left|f^{j}\left(I_{\beta}^{r}\right)\right| \frac{\sum_{i: f^{i}\left(I_{\alpha}^{n}\right) \subset f^{j}\left(I_{\beta}^{r}\right)}\left|f^{i}\left(I_{\alpha}^{n}\right)\right|}{\left|f^{j}\left(I_{\beta}^{r}\right)\right|} \\
= & \sum_{\beta \in \mathcal{A}} \sum_{j=1}^{q_{r}^{\beta}} \frac{D^{2} f\left(y_{j}^{\beta}\right)}{D f\left(y_{j}^{\beta}\right)} \cdot\left|f^{j}\left(I_{\beta}^{r}\right)\right| \cdot \ell\left((\alpha, \chi, n) \mid \omega_{j, \beta}\right) \\
& \pm \sum_{\beta \in \mathcal{A}} \sum_{j=1}^{q_{r}^{\beta}} \frac{D^{2} f\left(y_{j}^{\beta}\right)}{D f\left(y_{j}^{\beta}\right)} \cdot\left|f^{j}\left(I_{\beta}^{r}\right)\right| \cdot \ell((\alpha, 0, n)) \\
= & \sum_{\beta \in \mathcal{A}} \sum_{j=1}^{q_{r}^{\beta}} \frac{D^{2} f\left(y_{j}^{\beta}\right)}{D f\left(y_{j}^{\beta}\right)} \cdot\left|f^{j}\left(I_{\beta}^{r}\right)\right| \cdot\left[\ell\left((\alpha, \chi, n) \mid \omega_{j, \beta}\right)-\ell((\alpha, 0, n))\right] \\
& +\ell((\alpha, 0, n)) \cdot \sum_{\beta \in \mathcal{A}} \sum_{j=1}^{q_{r}^{\beta}} \frac{D^{2} f\left(y_{j}^{\beta}\right)}{D f\left(y_{j}^{\beta}\right)} \cdot\left|f^{j}\left(I_{\beta}^{r}\right)\right| \\
= & (\mathrm{III})+(\operatorname{IV}) .
\end{aligned}
$$

Pela Proposição 5.1.1, temos que (III) $=\mathrm{O}\left(\lambda_{4}^{\sqrt{r}}\right)$.

Sabemos que, 


$$
\int_{I} \frac{D^{2} f(s)}{D f(s)} d s=\sum_{\beta \in \mathcal{A}} \sum_{j=1}^{q_{r}^{\beta}} \frac{D^{2} f\left(z_{j}^{\beta}\right)}{D f\left(z_{j}^{\beta}\right)} \cdot\left|f^{j}\left(I_{\beta}^{r}\right)\right|,
$$

para $\operatorname{algum} z_{j}^{\beta} \in f^{j}\left(I_{\beta}^{r}\right)$. Então

$$
\begin{aligned}
|(\mathrm{IV})|= & \ell((\alpha, 0, n)) \cdot \sum_{\beta \in \mathcal{A}} \sum_{j=1}^{q_{r}^{\beta}} \frac{D^{2} f\left(y_{j}^{\beta}\right)}{D f\left(y_{j}^{\beta}\right)} \cdot\left|f^{j}\left(I_{\beta}^{r}\right)\right|+\ell((\alpha, 0, n)) \cdot \int_{I} \frac{D^{2} f(s)}{D f(s)} d s \\
& -\ell((\alpha, 0, n)) \cdot \sum_{\beta \in \mathcal{A}} \sum_{j=1}^{q_{r}^{\beta}} \frac{D^{2} f\left(z_{j}^{\beta}\right)}{D f\left(z_{j}^{\beta}\right)} \cdot\left|f^{j}\left(I_{\beta}^{r}\right)\right| \\
= & \ell((\alpha, 0, n)) \cdot \int_{I} \frac{D^{2} f(s)}{D f(s)} d s+\ell((\alpha, 0, n)) \cdot \sum_{\beta \in \mathcal{A}} \sum_{j=1}^{q_{r}^{\beta}}\left(\frac{D^{2} f\left(z_{j}^{\beta}\right)}{D f\left(z_{j}^{\beta}\right)}-\frac{D^{2} f\left(y_{j}^{\beta}\right)}{D f\left(y_{j}^{\beta}\right)}\right) \cdot\left|f^{j}\left(I_{\beta}^{r}\right)\right| \\
\leq & \ell((\alpha, 0, n)) \cdot \int_{I} \frac{D^{2} f(s)}{D f(s)} d s+C_{0} \cdot \lambda^{\frac{n}{2} \nu} .
\end{aligned}
$$

Em particular, se

$$
\int_{I} \frac{D^{2} f(x)}{D f(x)} d x=0
$$

então tomando $\lambda_{5}=\max \left\{\lambda, \lambda_{4}\right\}$ e $C_{25}=2 C_{0}+C_{23}$, obtemos pela Proposição 5.2.1 que

$$
\left|\int_{I_{\alpha}^{n}} \frac{D^{2} f_{n}(s)}{D f_{n}(s)} d s\right| \leq C_{25} \cdot \lambda_{5}^{\sqrt{\frac{n}{2}}}
$$

A prova do Teorema C, segue da Proposição 5.2.1, bastando o leitor notar que por definição

$$
\ell(\alpha, 0, n)=\frac{\sum_{i=1}^{q_{\alpha}^{n}}\left|f^{i}\left(I_{\alpha}^{n}\right)\right|}{|I|} .
$$

Para provar o Teorema D, precisamos do seguinte lema.

Lema 5.2.1. Sejam $a, b \in \mathbb{R}$. Então existe uma constante $C_{26}>0$ tal que

$$
\left|M_{a}-M_{b}\right|_{C^{2}} \leq C_{26} \cdot|a-b|
$$

onde $M_{a}$ e $M_{b}$ são definidas em (3.5).

Demonstração. Segue de (3.6) e (3.7). 
Demonstração. Teorema D: Como por hipótese

$$
\int_{I} \frac{D^{2} f(s)}{D f(s)} d s=0
$$

então pela Proposição 5.2.1 temos que para todo $\alpha \in \mathcal{A}$

$$
\left|\int_{I_{\alpha}^{n}} \frac{D^{2} f_{n}(s)}{D f_{n}(s)} d s\right| \leq C_{25} \cdot \lambda_{4}^{\sqrt{\frac{n}{2}}}
$$

Portanto, pelo Lema (5.2.1)

$$
\left\|M_{\int_{I_{\alpha}^{n}}^{n} \frac{D^{2} f_{n}(s)}{D f_{n}(s)} d s}-\mathrm{Id}\right\|_{C^{2}} \leq C_{26} \cdot\left|\int_{I_{\alpha}^{n}} \frac{D^{2} f_{n}(s)}{D f_{n}(s)} d s-0\right| \leq C_{25} \cdot C_{26} \cdot \lambda_{4}^{\sqrt{\frac{n}{2}}}
$$

para todo $\alpha \in \mathcal{A}$. O Teorema B junto com (5.4) nos dá

$$
\left\|Z_{I_{\alpha}^{n}}\left(R^{n}(f)\right)-\operatorname{Id}\right\|_{C^{2}} \leq C_{27} \cdot \lambda_{5}^{\sqrt{n}} \text { para todo } \alpha \in \mathcal{A},
$$

onde $\lambda_{5}=\lambda_{4}^{\frac{1}{\sqrt{2}}}$

Em particular a estimativa (5.5) nos diz que a função não linearidade de $R^{n}(f)$ vai pra zero, quando $n \rightarrow \infty$. 


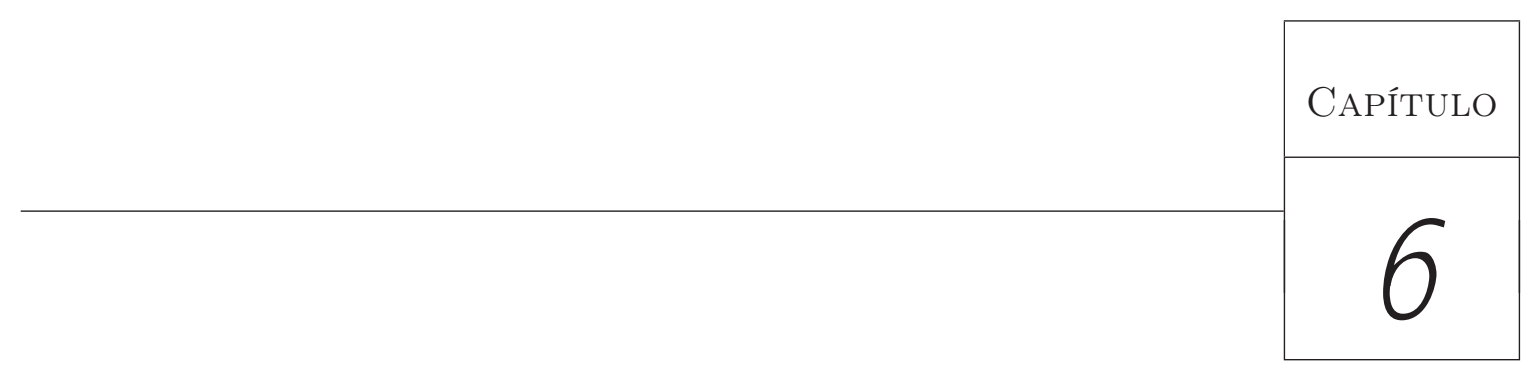

\section{Teoremas E e F}

Neste capítulo nós provaremos que dada uma $f \in \mathcal{B}_{k}^{2+\nu}$ nós conseguimos encontrar um modelo afim para $f$, ou seja, nós construímos uma t.i.i. afim $f_{A}$ que é conjugada a $f$ e só depende da combinatória de $f$ e das derivadas laterais de $f$ nos bordos dos intervalos $I_{\alpha}, \alpha \in \mathcal{A}$.

Teorema E. Seja $\left(f, \mathcal{A},\left\{I_{\alpha}\right\}_{\alpha \in \mathcal{A}}\right) \in \mathcal{B}_{k}^{2+\nu}$ e suponha que

$$
\int \frac{D^{2} f(x)}{D f(x)} d x=0
$$

Então existe uma t.i.i. $\left(f_{A}, \mathcal{A},\left\{\tilde{I}_{\alpha}\right\}_{\alpha \in \mathcal{A}}\right)$ e $0<\lambda<1$ tal que

(i) $\left.f_{A}\right|_{\tilde{I}_{\alpha}}$ é afim para cada $\alpha \in \mathcal{A}$ e tem a mesma combinatória de $f$;

(ii) $f_{A}$ é universal, isto é, se $f$ e g satisfazem as hipóteses do Teorema, tem a mesma combinatória e são break-equivalentes (Definição 1.1.3) então $f_{A}=g_{A}$.

(iii) $d_{C^{2}}\left(R^{n} f, R^{n} f_{A}\right)=\mathrm{O}\left(\lambda^{\sqrt{n}}\right)$.

Veja que se $f, g \in \mathcal{B}_{k}^{2+\nu}$ satisfazem as hipóteses do Teorema E, possuem a mesma combinatória e são break-equivalentes então por $(i i) f_{A}=g_{A}$ e portanto

$$
d_{C^{2}}\left(R^{n} f, R^{n} g\right)=\mathrm{O}\left(\lambda^{\sqrt{n}}\right) .
$$

Então temos o seguinte teorema que segue diretamente do Teorema E. 
Teorema F. Sejam $f, g \in \mathcal{B}_{k}^{2+\nu}$ tais que

- $f$ e g possuem a mesma combinatória;

- $f$ e g são break-equivalentes;

- $\int_{0}^{1} \frac{D^{2} f(s)}{D f(s)} d s=\int_{0}^{1} \frac{D^{2} g(s)}{D g(s)} d s=0$.

Então existe $0<\lambda<1$ tal que

$$
d_{C^{2}}\left(R^{n} f, R^{n} g\right)=\mathrm{O}\left(\lambda^{\sqrt{n}}\right)
$$

Fixada a combinatória de $f$, sabemos pela equação (2.8) que bsta encontrar um vetor derivada que é uma órbita do cociclo de Rauzy-Veech. Para isto mostraremos que o cociclo de Rauzy-Veech é parcialmente hiperbólico em $\mathbb{R}^{\mathcal{A}}$ e ademais se comporta como uma quasi-isometria na sua direção central.

\subsection{Hiperbolicidade Uniforme}

Seja $\Omega_{\pi}: \mathbb{R}^{\mathcal{A}} \rightarrow \mathbb{R}^{\mathcal{A}}$ definida em (2.12). Se $\pi$ é de genus 1 , sabemos que dim Ker $\Omega_{\pi}=$ $d-2$ e $\operatorname{dim} \operatorname{Im} \Omega_{\pi}=2$.

Lembramos, que pelo Lema 2.5.1, os operadores $\Theta_{\pi, \varepsilon}$ e $\Omega_{\pi}$ satisfazem a seguinte relação

$$
\Theta_{\pi, \varepsilon} \Omega_{\pi}=\Omega_{\pi^{\prime}}{ }^{t} \Theta_{\pi, \varepsilon}^{-1}
$$

onde $\pi^{\prime}=r_{\varepsilon}(\pi)$.

Nós provaremos que o cociclo de Rauzy-Veech é hiperbólico na $\operatorname{Im} \Omega$.

\subsubsection{Cones Invariantes}

Desde que $\Theta_{\pi, \varepsilon}$ e ${ }^{t} \Theta_{\pi, \varepsilon}$ são matrizes não negativas, elas preservam o cone positivo $\mathbb{R}_{+}^{\mathcal{A}}$. Pelo Corolário 2.5.1 temos que

$$
\begin{gathered}
\Theta_{\pi, \varepsilon} \operatorname{Im} \Omega_{\pi}=\operatorname{Im} \Omega_{\pi^{\prime}}, \\
{ }^{t} \Theta_{\pi, \varepsilon} \operatorname{Ker} \Omega_{\pi}=\operatorname{Ker} \Omega_{\pi^{\prime}} .
\end{gathered}
$$

Dada uma permutação irredutível $\pi=\left(\pi_{0}, \pi_{1}\right)$ e um vetor $\tau \in \mathbb{R}^{\mathcal{A}}$, defina $h \in \mathbb{R}^{\mathcal{A}}$ por

$$
h_{\alpha}=-\sum_{\pi_{1}(\beta)<\pi_{1}(\alpha)} \tau_{\beta}+\sum_{\pi_{0}(\beta)<\pi_{0}(\alpha)} \tau_{\beta}=-\Omega_{\pi}(\tau)_{\alpha} .
$$


Observe que se $\tau \in T_{\pi}^{+}$então

$$
\sum_{\pi_{0}(\beta)<\pi_{0}(\alpha)} \tau_{\beta}>0>\sum_{\pi_{1}(\beta)<\pi_{1}(\alpha)} \tau_{\beta} \Rightarrow h_{\alpha}>0
$$

para todo $\alpha \in \mathcal{A}$.

Agora nós definiremos cones dentro de $\operatorname{Im} \Omega_{\pi}$ que são invariantes pela ações de $\Theta_{\pi, \varepsilon}$ e $\Theta_{\pi, \varepsilon}^{-1}$. Definimos

$$
\begin{gathered}
C_{\pi}^{s}:=\Omega_{\pi} \mathbb{R}_{+}^{\mathcal{A}} \subset \operatorname{Im} \Omega_{\pi} \\
C_{\pi}^{u}=-\Omega_{\pi} T_{\pi}^{+} \subset \operatorname{Im} \Omega_{\pi} .
\end{gathered}
$$

O seguinte Lema pode ser encontrado em [Via08, Lemma 2.13].

Lema 6.1.1. A transformação linear ${ }^{t} \Theta_{\pi, \varepsilon}^{-1}$ leva $T_{\pi}^{+}$injetivamente dentro de $T_{\pi^{\prime}}^{+}$. Mais precisamente, a imagem coincide com o conjunto dos vetores $\tau^{\prime} \in T_{\pi^{\prime}}^{+}$cujo tipo é $1-\varepsilon$.

Lema 6.1.2. Os cones $C_{\pi}^{s}$ e $C_{\pi}^{u}$ são invariantes por $\Theta_{\pi, \varepsilon}^{-1}$ e $\Theta_{\pi, \varepsilon}$, respectivamente. Ou seja

$$
\Theta_{\pi, \varepsilon}^{-1} C_{\pi^{\prime}}^{s} \subset C_{\pi}^{s} \quad e \Theta_{\pi, \varepsilon} C_{\pi}^{u} \subset C_{\pi^{\prime}}^{u}
$$

Demonstração. Por (6.2) e sabendo que ${ }^{t} \Theta_{\pi, \varepsilon}\left(\mathbb{R}_{+}^{\mathcal{A}}\right) \subset \mathbb{R}_{+}^{\mathcal{A}}$, temos

$$
C_{\pi^{\prime}}^{s}=\Omega_{\pi^{\prime}}\left(\mathbb{R}_{+}^{\mathcal{A}}\right)=\Theta_{\pi, \varepsilon} \Omega_{\pi}^{t} \Theta_{\pi, \varepsilon}\left(\mathbb{R}_{+}^{\mathcal{A}}\right) \subset \Theta_{\pi, \varepsilon} \Omega_{\pi}\left(\mathbb{R}_{+}^{\mathcal{A}}\right)=\Theta_{\pi, \varepsilon} C_{\pi}^{s}
$$

Novamente por (6.2) e pelo Lema 6.1.1, temos

$$
C_{\pi^{\prime}}^{u}=-\Omega_{\pi^{\prime}}\left(T_{\pi^{\prime}}^{+}\right)=-\Theta_{\pi, \varepsilon} \Omega_{\pi}^{t} \Theta_{\pi, \varepsilon}\left(T_{\pi^{\prime}}^{+}\right) \supset-\Theta_{\pi, \varepsilon} \Omega_{\pi}\left(T_{\pi}^{+}\right)=\Theta_{\pi, \varepsilon} C_{\pi}^{u}
$$

Proposição 6.1.1 (Hiperbolicidade Uniforme). Seja $\pi^{0}, \pi^{1}, \ldots$ uma sequência em $\Pi^{1}$, com $r_{\varepsilon^{n}}\left(\pi^{n}\right)=\pi^{n+1}$ tal que $\left\{\pi^{i}, \varepsilon^{i}\right\}_{i \in \mathbb{N}}$ é $k$-limitado. Então existem constantes $\mu=\mu(k)>1$ e $C_{i}=C_{i}(k)>0$ com $i \in\{s, u\}$, tal que para cada $n \in \mathbb{N}$

A. $\left\|\left(\Theta_{\pi^{n}, \varepsilon^{n}} \cdots \Theta_{\pi^{1}, \varepsilon^{1}} \Theta_{\pi^{0}, \varepsilon^{0}}\right) v\right\| \geq C_{u} \mu^{n}\|v\| \forall v \in C_{\pi^{0}}^{u}$,

B. $\left\|\left(\Theta_{\pi^{n-1}, \varepsilon^{n-1}} \cdots \Theta_{\pi^{0}, \varepsilon^{0}}\right)^{-1} v\right\| \geq C_{s} \mu^{n}\|v\| \forall v \in C_{\pi^{n}}^{s}$.

Demonstração. Em [MMY05a, Seção 1.2.4] os autores mostram que se uma sequência qualquer de combintória $\left\{\left(\pi^{0}, \varepsilon^{0}\right), \ldots,\left(\pi^{j}, \varepsilon^{j}\right)\right\}$ é $(2 d-3)$-completo (veja Seção 2.3, para definição) então

$$
\Theta_{0, i}:=\Theta_{\pi^{i}, \varepsilon^{i}} \cdots \Theta_{\pi^{0}, \varepsilon^{0}}>0
$$


Como a combinatória $\left\{\pi^{i}, \varepsilon^{i}\right\}_{i \in \mathbb{N}}$ é $k$-limitado, temos que qualquer sequência finita $\left\{\pi^{i}, \varepsilon^{i}\right\}_{i=n}^{i=n+k}$ é completa. Pelo exposto acima, temos para todo $n \in \mathbb{N}$

$$
\Theta_{n, n+k(2 d-3)}=\Theta_{\pi^{n+k(2 d-3)}, \varepsilon^{n+k(2 d-3)}} \cdots \Theta_{\pi^{n+1}, \varepsilon^{n+1}} \Theta_{\pi^{n}, \varepsilon^{n}}>0 .
$$

Em [Yoc, Seção 10.3] o autor mostra que se uma sequência $\left\{\left(\pi^{0}, \varepsilon^{0}\right), \ldots,\left(\pi^{j}, \varepsilon^{j}\right)\right\}$ é $3 d-4$-completa, então

$$
{ }^{t}\left(\Theta_{0, j}\right)^{-1}:={ }^{t}\left(\Theta_{\pi^{j}, \varepsilon^{j}} \cdots \Theta_{\pi^{0}, \varepsilon^{0}}\right)^{-1}\left(\overline{T_{\pi^{0}}^{+}}\right) \subset T_{\pi^{j+1}}^{+} \cup\{0\} .
$$

Do exposto acima e do fato de que $\Theta_{\pi^{j}, \varepsilon^{j}} \overline{\Omega_{\pi^{j}}\left(T_{\pi^{j}}^{+}\right)}=\Omega_{\pi^{j+1}} \overline{t \Theta_{\pi^{j}, \varepsilon^{j}}^{-1}\left(T_{\pi^{j}}^{+}\right)}$, obtemos

$$
\Theta_{n, n+k(3 d-4)} \overline{C_{\pi^{n}}^{u}} \subset C_{\pi^{n+k(3 d-4)+1}}^{u} \cup\{0\}
$$

para todo $n \in \mathbb{N}$, e

$$
\left\|\Theta_{n, n+k(3 d-4)} v\right\|_{1} \geq d\|v\|_{1},
$$

para todo $v \in C_{\pi^{n}}^{u}$. Para todo $n \in \mathbb{N}$, temos $n=q k(3 d-4)+r$, com $q, r \in \mathbb{N}$ e $0 \leq r<k(3 d-4)$. Então

$$
\begin{aligned}
\left\|\Theta_{0, n} v\right\| & \geq d^{q}\left\|\Theta_{0, r} v\right\|_{1} \geq d^{(n-r) /(k(3 d-4)} \min _{0 \leq r<k(3 d-4)}\left\{\left\|\Theta_{0, r}^{-1}\right\|^{-1}\right\}\|v\|_{1} \\
& \geq C_{u} \mu^{n}\|v\|_{1},
\end{aligned}
$$

o que prova a afirmação A.

Agora provaremos a afirmação B. Usando argumentos similares da prova da afirmação A, podemos mostrar

$$
\left\|^{t}\left(\Theta_{\pi^{n}, \varepsilon^{n}} \cdots \Theta_{\pi^{0}, \varepsilon^{0}}\right) w\right\| \geq C_{\mu} \mu^{n}\|w\|,
$$

para todo $w \in \mathbb{R}_{+}^{\mathcal{A}}$. De $(6.2)$, obtemos

$$
\left(\Theta_{\pi^{n-1}, \varepsilon^{n-1}} \cdots \Theta_{\pi^{0}, \varepsilon^{0}}\right)^{-1} \Omega_{\pi^{n}}=\Omega_{\pi^{0}}{ }^{t}\left(\Theta_{\pi^{n-1}, \varepsilon^{n-1}} \cdots \Theta_{\pi^{0}, \varepsilon^{0}}\right) .
$$

O fato de que ${ }^{t} \Theta_{i, i+k(3 d-4)}$, para todo $i \in \mathbb{N}$, implica que existem $\delta_{1}, \delta_{2} \in(0,1)$ tal que

$$
{ }^{t} \Theta_{0, n} \mathbb{R}_{+}^{\mathcal{A}} \subset \Lambda_{\delta_{1}, \delta_{2}}=\left\{\left(\lambda_{\alpha}\right)_{\alpha \in \mathcal{A}} \in \mathbb{R}_{+}^{\mathcal{A}}, \delta_{1} \leq \frac{\lambda_{\alpha}}{\sum_{\beta} \lambda_{\beta}} \leq \delta_{2}\right\} .
$$

Afirmamos que

$$
\mathbb{R}_{+}^{\mathcal{A}} \cap \operatorname{Ker} \Omega_{\pi^{n}}=\emptyset
$$

De fato, seja $\lambda \in \mathbb{R}_{+}^{\mathcal{A}}$ tal que $\Omega_{\pi^{n}}(\lambda)=0$. Então pela definição de $\Omega_{\pi^{n}}$ temos que

$$
\sum_{\pi_{1}(\beta)<\pi_{1}(\alpha)} \lambda_{\beta}-\sum_{\pi_{0}(\beta)<\pi_{0}(\alpha)} \lambda_{\beta}=0, \text { para todo } \alpha \in \mathcal{A} .
$$


Seja $\alpha_{0} \in \mathcal{A}$ tal que $\pi^{0}\left(\alpha_{0}\right)=d$. Então

$$
\begin{aligned}
0 & =\sum_{\pi_{1}(\beta)<\pi_{1}\left(\alpha_{0}\right)} \lambda_{\beta}-\sum_{\pi_{0}(\beta)<d} \lambda_{\beta}=0 \\
& =\sum_{\pi_{1}(\beta)<\pi_{1}\left(\alpha_{0}\right)} \lambda_{\beta}-\sum_{\pi_{1}(\beta)<\pi_{1}\left(\alpha_{0}\right)} \lambda_{\beta}-\sum_{\pi_{1}(\beta)>\pi_{1}\left(\alpha_{0}\right)} \lambda_{\beta} \\
& =-\sum_{\pi_{1}(\beta)>\pi_{1}\left(\alpha_{0}\right)} \lambda_{\beta} .
\end{aligned}
$$

Temos que $\sharp\left\{\beta \in \mathcal{A}: \pi_{1}(\beta)>\pi_{1}\left(\alpha_{0}\right)\right\} \geq 1$, pois caso contrário, $\pi_{1}\left(\alpha_{0}\right)=d$, o que é impossível, pois $\pi=\left(\pi_{0}, \pi_{1}\right)$ é irredutível. Mas isto contradiz (6.11).

De (6.10) temos que existe constante $Q>0$, tal que

$$
Q=\inf \left\{\frac{\left|\Omega_{\pi^{n}} u\right|_{1}}{|u|_{1}}, u \neq 0, u \in \Lambda_{\delta_{1}, \delta_{2}}\right\}
$$

Para todo $v \in C_{\pi^{n}}^{s}$ existe $w \in \mathbb{R}_{+}^{\mathcal{A}}$ tal que

$$
\begin{aligned}
\left\|\left(\Theta_{\pi^{n-1}, \varepsilon^{n-1}} \cdots \Theta_{\pi^{0}, \varepsilon^{0}}\right)^{-1} v\right\| & =\left\|\Omega_{\pi^{0}} t\left(\Theta_{\pi^{n-1}, \varepsilon^{n-1}} \cdots \Theta_{\pi^{0}, \varepsilon^{0}}\right) w\right\| \\
& \geq Q \cdot\left\|^{t}\left(\Theta_{\pi^{n-1}, \varepsilon^{n-1}} \cdots \Theta_{\pi^{0}, \varepsilon^{0}}\right) w\right\| \\
& \geq Q \cdot C_{\mu} \cdot \mu^{n-1}\|w\| \\
& \geq Q \cdot C_{\mu} \cdot \frac{1}{S} \cdot \mu^{n-1}\|v\|,
\end{aligned}
$$

onde

$$
S=\sup _{\pi \in \Pi^{1}} \sup _{v \in \mathbb{R}^{\mathcal{A}}}\left\{\frac{\left\|\Omega_{\pi} v\right\|}{\|v\|}\right\}
$$

Motivado pelo resultado da Proposição 6.1.1, definimos a direção estável do ponto $\left\{\pi^{j}, \varepsilon^{j}\right\}$ como sendo

$$
E_{j}^{s}:=E^{s}\left(\pi^{j}\right)=\bigcap_{n \geq 0} \Theta_{j}^{-1} \cdots \Theta_{j+n}^{-1}\left(C_{\pi^{j+n+1}}^{s}\right) .
$$

É fácil ver que os subespaços definidos acima, são invariante pelo cociclo de RauzyVeech, ou seja, para todo $j \geq 0$

$$
\Theta_{j}\left(E_{j}^{s}\right)=E_{j+1}^{s}
$$

Agora definiremos a direção instável. Seja $u_{0} \in C_{\pi^{0}}^{u}$, tal que $\left\|u_{0}\right\|=1$. Então defina $E_{0}^{u}$ como sendo o subespaço gerado pelo vetor $u_{0}$, que denotaremos por $\left\langle u_{0}>\right.$. Agora para todo $j>0$ definimos

$$
E_{j}^{u}:=<\frac{u_{j}}{\left\|u_{j}\right\|}>\text {, onde } u_{j}=\Theta_{j-1}\left(u_{j-1}\right)
$$


Por definição os subespaços $E_{j}^{u}$ são invariantes pelo cocilco de Rauzy-Veech.

Mostraremos na próxima seção que fora de $\operatorname{Im} \Omega$ o cociclo de Rauzy-Veech possui uma direção central. Além disso, mostraremos que o mesmo se comporta como uma quase-isometria nesta direção.

\subsection{Direção Central}

Seja $p \in \mathbb{N}$ tal que $\left\{\pi^{0}, \varepsilon^{0}\right\}=\left\{\pi^{p}, \varepsilon^{p}\right\}$ e

$$
\Theta_{0, p-1}=\Theta_{\pi^{p-1}, \varepsilon^{p-1}} \cdots \Theta_{\pi^{0}, \varepsilon^{0}} .
$$

Denote por $E_{0, p-1}^{c}$ o espaço central associado a $\Theta_{0, p-1}$. No próximo Lema, é obtido uma caracterização para $E_{0, p-1}^{c}$.

Lema 6.2.1. $E_{0, p-1}^{c}=\left\{k+\Psi_{p}(k), k \in \operatorname{Ker} \Omega_{\pi^{0}}\right\}$, onde $\Psi_{p}(k)=\left(\Theta_{0, p-1}-\mathrm{Id}\right)^{-1}(k-$ $\left.\Theta_{0, p-1}(k)\right)$.

Demonstração. Dado qualquer $v \in E_{0, p-1}^{c}$, podemos escrever $v=i+k$, onde $i \in \operatorname{Im} \Omega_{\pi^{0}}$ e $k \in \operatorname{Ker} \Omega_{\pi^{0}}$. Assim,

$$
\Theta_{0, p-1}(i+k)=i+k \Longrightarrow\left(\Theta_{0, p-1}-\mathrm{Id}\right)(i)=k-\Theta_{0, p-1}(k) .
$$

Afirmamos que $k-\Theta_{0, p-1}(k) \in \operatorname{Im} \Omega_{\pi^{0}}$, ou equivalentemente, $k-\Theta_{0, p-1}(k) \perp$ Ker $\Omega_{\pi^{0}}$. De fato, pelo Lema 2.5.2, temos que $\left.\left(\Theta_{0, p-1}^{t}\right)^{-1}\right|_{\text {Ker } \Omega_{\pi^{0}}}=$ Id. Logo, para todo $u \in \operatorname{Ker} \Omega_{\pi^{0}}$

$$
\begin{aligned}
<u, k-\Theta_{0, p-1}(k)> & =<u, k>-<\Theta_{0, p-1}^{t}(u), k> \\
& =<u, k>-<u, k>=0 .
\end{aligned}
$$

Portanto $k-\Theta_{0, p-1}(k) \perp \operatorname{Ker} \Omega_{\pi^{0}}$, como afirmamos. Então

$$
i=\left(\Theta_{0, p-1}-\mathrm{Id}\right)^{-1}\left(k-\Theta_{0, p-1}(k)\right)=\Psi_{p}(k) .
$$

Suponhamos agora, que nossa combinatória é periódica, ou seja, existe $p \in \mathbb{N}$ tal que para todo $n \in \mathbb{N}$

$$
\left\{\pi^{n}, \varepsilon^{n}\right\}=\left\{\pi^{n+p}, \varepsilon^{n+p}\right\} .
$$

O Lema 6.2.1 nos diz que $\operatorname{dim} E_{0, p-1}^{c}=\operatorname{dim} \operatorname{Ker} \Omega_{\pi^{0}}=d-2$. Nosso próximo resultado, mostra a invariância de $E_{0, p-1}^{c}$. 
Lema 6.2.2. Nas condições mencionadas acima, temos

$$
\Theta_{\pi^{0}, \varepsilon^{0}}\left(E_{0, p-1}^{c}\right)=E_{1, p}^{c}
$$

Demonstração. Seja $v \in E_{0, p-1}^{c}$. Então

$$
\Theta_{\pi^{p-1}, \varepsilon^{p-1}} \cdots \Theta_{\pi^{0}, \varepsilon^{0}}(v)=v .
$$

Aplicando $\Theta_{\pi^{0}, \varepsilon^{0}}$ em ambos os lados na equação acima,

$$
\Theta_{\pi^{0}, \varepsilon^{0}} \cdot \Theta_{\pi^{p-1}, \varepsilon^{p-1}} \cdots \Theta_{\pi^{1}, \varepsilon^{1}} \cdot \Theta_{\pi^{0}, \varepsilon^{0}}(v)=\Theta_{\pi^{0}, \varepsilon^{0}}(v) .
$$

Assim

$$
\Theta_{1, p} \cdot \Theta_{\pi^{0}, \varepsilon^{0}}(v)=\Theta_{\pi^{0}, \varepsilon^{0}}(v) \Rightarrow \Theta_{\pi^{0}, \varepsilon^{0}}(v) \in E_{1, p}^{c} .
$$

Agora mostraremos que o cociclo de Zorich se comporta basicamente como uma quasi-isometria na direção central. Pela Proposição 6.1.1, podemos escolher $n_{0}>0 \mathrm{e}$ constante $\mu>1$, tal que

$$
\left\|\Theta_{n, n+n_{0}-1}(x)\right\| \geq \mu\|x\| \forall x \in C_{\pi^{n}}^{u} \quad \text { and } \quad\left\|\Theta_{n+n_{0}-1, n}(y)\right\| \geq \mu\|y\| \forall y \in C_{\pi^{n+n_{0}}}^{s} .
$$

Defina para cada $\epsilon>0$, o cone $C_{\epsilon}^{n}=C_{\epsilon, u}^{n} \dot{\cup} C_{\epsilon, s}^{n}$, onde $C_{\epsilon, u}^{n}$ é o conjunto de vetores $x=x_{k}+x_{i} \in \operatorname{Ker} \Omega_{\pi^{n}} \oplus \operatorname{Im} \Omega_{\pi^{n}}$ tal que

- $\left\|x_{k}\right\| \leq \epsilon\left\|x_{i}\right\|$

- $\exists x_{i}^{s} \in \Theta_{n+n_{0}-1, n} C_{\pi^{n+n_{0}}}^{s} \subset C_{\pi^{n}}^{s}$ e $\exists x_{i}^{u} \in \Theta_{n-n_{0}, n-1} C_{\pi^{n-n_{0}}}^{u} \subset C_{\pi^{n}}^{u}$ tal que $x_{i}=x_{i}^{s}+x_{i}^{u}$ e $\left\|x_{i}^{s}\right\| \leq\left\|x_{i}^{u}\right\|$.

Analogamente definimos $C_{\epsilon, s}^{n}$ mudando a última condição para $\left\|x_{i}^{u}\right\| \leq\left\|x_{i}^{s}\right\|$.

Proposição 6.2.1. Existe $\epsilon_{0}=\epsilon_{0}(k)>0$ e $\gamma<1$ tal que se $\epsilon<\epsilon_{0}$ então

$$
\Theta_{n, n+n_{0}-1}\left(C_{\epsilon, u}^{n}\right) \subset C_{\gamma \epsilon, u}^{n+n_{0}} \text { and } \Theta_{n-1, n-n_{0}}\left(C_{\epsilon, s}^{n}\right) \subset C_{\gamma \epsilon, s}^{n-n_{0}} .
$$

Antes de provar a Proposição 6.2.1, precisaremos de dois lemas.

Tomando $C_{28}=C_{28}\left(n_{0}\right):=\max \underset{\substack{\pi \in \Pi_{d} \\ \varepsilon \in\{0,1\}}}{ }\left\{\left\|\prod_{i=0}^{n_{0}-1} \Theta_{\pi^{i}, \varepsilon^{i}}\right\|\right\}$ temos:

Lema 6.2.3. Para todo $v \in C_{\pi^{n+n_{0}}}^{s}$, existe $w \in \Theta_{n+2 n_{0}-1, n+n_{0}}\left(C_{\pi^{n+2 n_{0}}}^{s}\right)$ satisfazendo

$$
\|w\| \leq C_{28}\|v\|
$$


Lema 6.2.4. Para todo $n>n_{0}$ e para todo $x_{k} \in \operatorname{Ker} \Omega_{\pi^{n}}$

$$
\begin{aligned}
\left\|\Theta_{n, n+n_{0}-1}\left(x_{k}\right)\right\|_{\operatorname{Ker} \Omega_{\pi^{n+n_{0}}}}:=\sup _{k_{n+n_{0}} \in \operatorname{Ker} \Omega_{\pi^{n+n_{0}}}}<\Theta_{n, n+n_{0}-1}\left(x_{k}\right), k_{n+n_{0}}> \\
=\left\|x_{k}\right\|_{\operatorname{Ker} \Omega_{\pi^{n}}},
\end{aligned}
$$

onde $\left\langle\cdot, \cdot>\right.$ denota o produto interno usual do $\mathbb{R}^{\mathcal{A}}$.

Demonstração. Note quet ${ }^{t} \Theta_{n, n+n_{0}-1}\left(\operatorname{Ker} \Omega_{\pi^{n+n_{0}}}\right)=\operatorname{Ker} \Omega_{\pi^{n}}$. Portanto,

$$
\begin{aligned}
& \sup _{k_{n+n_{0}} \in \operatorname{Ker} \Omega_{\pi^{n+n_{0}}}<\Theta_{n, n+n_{0}-1}\left(x_{k}\right), k_{n+n_{0}}>=}=\sup _{k_{n+n_{0}} \in \operatorname{Ker} \Omega_{\pi^{n+n_{0}}}<x_{k},{ }^{t} \Theta_{n, n+n_{0}-1}\left(k_{n+n_{0}}\right)>} \quad \sup _{k_{n} \in \operatorname{Ker} \Omega_{\pi^{n}}}<x_{k}, k_{n}> \\
& =\left\|x_{k}\right\|_{\text {Ker } \Omega_{\pi^{n}} .}
\end{aligned}
$$

Demonstração da Proposição 6.2.1. Seja $x=x_{i}+x_{k} \in C_{\epsilon, u}^{n}$. Primeiro note que,

$$
\begin{aligned}
\left\|\Theta_{n, n+n_{0}-1}\left(x_{i}\right)\right\| & =\left\|\Theta_{n, n+n_{0}-1}\left(x_{i}^{u}+x_{i}^{s}\right)\right\| \\
& \geq \mu\left\|x_{i}^{u}\right\|-\frac{1}{\mu} C_{28}\left\|x_{i}^{s}\right\| \\
& \geq\left(\mu-\frac{1}{\mu} C_{28}\right)\left\|x_{i}^{u}\right\|,
\end{aligned}
$$

e que

$$
\begin{aligned}
\left\|\Theta_{n, n+n_{0}-1}\left(x_{k}\right)\right\|_{\operatorname{Im} \Omega_{\pi^{n+n_{0}}}} & \leq\left\|\Theta_{n, n+n_{0}-1}\left(x_{k}\right)\right\| \\
& \leq\left\|\Theta_{n, n+n_{0}-1}\right\| \cdot\left\|x_{k}\right\| \\
& \leq \epsilon \cdot\left\|\Theta_{n, n+n_{0}-1}\right\| \cdot\left\|x_{i}\right\| .
\end{aligned}
$$

Agora notemos que pelo Lema 6.2.4 nós temos

$$
\begin{aligned}
\left\|\Theta_{n, n+n_{0}-1}\left(x_{k}\right)\right\|_{\text {Ker } \Omega_{\pi^{n+n_{0}}}} & =\left\|x_{k}\right\|_{\text {Ker } \Omega_{\pi^{n}}} \leq \epsilon\left\|x_{i}\right\| \\
& \leq 2 \epsilon\left\|x_{i}^{u}\right\| .
\end{aligned}
$$

Então 


$$
\begin{aligned}
& \left\|\Theta_{n, n+n_{0}-1}(x)\right\|_{\operatorname{Im} \Omega_{\pi^{n+n_{0}}}} \\
& \quad \geq\left\|\Theta_{n, n+n_{0}-1}\left(x_{i}\right)\right\|_{\operatorname{Im} \Omega_{\pi^{n+n_{0}}}}-\left\|\Theta_{n, n+n_{0}-1}\left(x_{k}\right)\right\|_{\operatorname{Im} \Omega_{\pi^{n+n_{0}}}} \\
& \quad \geq\left(\mu-\frac{1}{\mu} C_{28}\right)\left\|x_{i}^{u}\right\|-2 \epsilon \cdot\left\|\Theta_{n, n+n_{0}-1}\right\| \cdot\left\|x_{i}^{u}\right\|, \text { por }(6.14) \text { e }(6.15) \\
& \quad \geq \frac{1}{2 \epsilon}\left(\mu-\frac{1}{\mu} C_{28}-2 \epsilon \cdot\left\|\Theta_{n, n+n_{0}-1}\right\|\right)\left\|\Theta_{n, n+n_{0}-1}\left(x_{k}\right)\right\|_{\operatorname{Ker} \Omega_{\pi^{n+n}}} \text { por }(6.16) \\
& \quad \geq \frac{1}{2 \epsilon}\left(\mu-\frac{1}{\mu} C_{28}-2 \epsilon \cdot\left\|\Theta_{n, n+n_{0}-1}\right\|\right)\left\|\Theta_{n, n+n_{0}-1}(x)\right\|_{\operatorname{Ker} \Omega_{\pi^{n+n_{0}}}} .
\end{aligned}
$$

Portanto,

$$
\begin{aligned}
& \left\|\Theta_{n, n+n_{0}-1}(x)\right\|_{\text {Ker } \Omega_{\pi^{n+n_{0}}}} \\
& \quad \leq 2 \epsilon\left(\mu-\frac{1}{\mu} C_{28}-2 \epsilon \cdot\left\|\Theta_{n, n+n_{0}-1}\right\|\right)^{-1}\left\|\Theta_{n, n+n_{0}-1}(x)\right\|_{\operatorname{Im} \Omega_{\pi^{n+n_{0}}}} .
\end{aligned}
$$

Tomando $\mu>1+\sqrt{1+C_{28}}$ e $\epsilon=\frac{1}{2\left\|\Theta_{n, n+n_{0}-1}\right\|}\left(\mu-\frac{1}{\mu} C_{28}-2\right)$, nós obtemos o resultado.

Proposição 6.2.2. Seja $p>n_{0}$ tal que $\pi^{p+n}=\pi^{n}$ para todo $n$. Então existe $\epsilon_{0}>0$ tal que

$$
\sup _{\substack{k \in \operatorname{Ker} \Omega_{\pi^{0}} \\ k \neq 0}} \frac{\left\|\Psi_{p}(k)\right\|}{\|k\|} \leq \frac{1}{\epsilon_{0}} .
$$

Demonstração. Suponha que a proposição é falsa. Então podemos encontrar $\epsilon<\epsilon_{0}$ tal que

$$
\sup _{\substack{k \in \operatorname{Ker} \Omega \\ k \neq 0}} \frac{\left\|\Psi_{p}(k)\right\|}{\|k\|}=\frac{1}{\epsilon}>\frac{1}{\epsilon_{0}} .
$$

Seja $k_{0} \in \operatorname{Ker} \Omega_{\pi^{0}}$ tal que o supremo é atingido. Temos que $k_{0}+\Psi_{p}\left(k_{0}\right) \in C_{\epsilon}^{0}=$ $C_{\epsilon, u}^{0} \dot{\cup} C_{\epsilon, s}^{0}$. Assuma, sem perda de generalidade, que $k_{0}+\Psi_{p}\left(k_{0}\right) \in C_{\epsilon, u}^{0}$. Por hipótese $E_{0, p-1}^{c}=E_{n_{0} p,\left(n_{0}+1\right) p-1}^{c}$ e $\Theta_{0, n_{0} p-1}\left(E_{0, p-1}^{c}\right)=E_{n_{0} p,\left(n_{0}+1\right) p-1}^{c}$, pelo Lema 6.2.2. Assim, pela Proposição 6.2.1

$$
\begin{gathered}
\tilde{k}_{0}+\Psi_{p}\left(\tilde{k}_{0}\right)=\Theta_{0, n_{0} p-1}\left(k_{0}+\Psi_{p}\left(k_{0}\right)\right) \in C_{\gamma \epsilon, u}^{n_{0} p} \Longleftrightarrow \\
\left\|\tilde{k}_{0}\right\| \leq \gamma \epsilon\left\|\Psi_{p}\left(\tilde{k}_{0}\right)\right\| \Longleftrightarrow \frac{\left\|\Psi_{p}\left(\tilde{k}_{0}\right)\right\|}{\left\|\tilde{k}_{0}\right\|} \geq \frac{1}{\gamma \epsilon}>\frac{1}{\epsilon},
\end{gathered}
$$

o que contradiz (6.19). 
Seja $f \in \mathcal{B}_{k, *}^{2+\nu}$ e $\gamma(f)=\left\{\pi^{i}, \varepsilon^{i}\right\}_{i \in \mathbb{N}}$ sua combinatória. Para cada $n \in \mathbb{N}$ defina a seguinte combinatória periódica $\gamma_{n}(f)=\left\{\tilde{\pi}^{i}, \tilde{\varepsilon}^{i}\right\}_{i \in \mathbb{N}}$ para $f$ :

(a) Para $i \leq n$ defina $\left(\tilde{\pi}^{i}, \tilde{\varepsilon}^{i}\right)=\left(\pi^{i}, \varepsilon^{i}\right)$, e denote esse caminho por $\gamma_{n}$;

(b) Seja $\tilde{\gamma}$ um caminho orientado ligando $\left(\pi^{n}, \varepsilon^{n}\right)$ a $\left(\pi^{0}, \varepsilon^{0}\right)$. A existência desse caminho é garantido em [Via08].

Seja $\tilde{\gamma}_{n}=\gamma_{n} * \tilde{\gamma}$, onde $*$ é a operação de justaposição. Agora defina a seguinte combinatória periódica de período $p_{n}$

$$
\gamma_{n}(f):=\tilde{\gamma}_{n} * \tilde{\gamma}_{n} * \cdots * \tilde{\gamma}_{n} * \cdots
$$

O cocilo de Rauzy-Veech associado a $\gamma_{n}(f)$ será denotado por $\tilde{\Theta}$.

É claro que $\gamma_{n}(f) \rightarrow \gamma(f)$ quando $n \rightarrow \infty$ e $\Theta_{j}=\tilde{\Theta}_{j}$ se e somente se $j \leq n$.

Pelos Lemas 6.2.1 e 6.2.2 temos que para todo $s \geq 0$ o subespaço $E_{s, p_{n}}^{c}$ é o gráfico da aplicação $\Psi_{s, p_{n}}$ e $\Theta_{s}\left(E_{s, p_{n}}^{c}\right)=E_{s+1, p_{n}}^{c}$. Pela Proposição 6.2 .2 a sequência $\left\{\Psi_{0, p_{n}}\right\}_{n \in \mathbb{N}}$ é equicontínua e equilimitada, logo possui uma subsequência uniformemente convergente $\left\{\Psi_{0, p_{n}}\right\}_{n \in \mathbb{N}_{0}}$. Igualmente, a sequência $\left\{\Psi_{1, p_{n}}\right\}_{n \in \mathbb{N}_{0}}$ é equicontínua e equilimitada, logo podemos encontrar um subconjunto infinito $\mathbb{N}_{1} \subset \mathbb{N}_{0}$ tal que $\left\{\Psi_{1, p_{n}}\right\}_{n \in \mathbb{N}_{1}}$ seja convergente. Prosseguindo analogamente, conseguimos, para cada $j \in \mathbb{N}$ um subconjunto infinito $\mathbb{N}_{j} \subset \mathbb{N}$, de tal modo que $\mathbb{N}_{0} \supset \mathbb{N}_{1} \supset \cdots \supset \mathbb{N}_{j} \supset \cdots$ e $\left\{\Psi_{j, p_{n}}\right\}_{n \in \mathbb{N}_{j}}$ converge uniformemente para $\Psi_{j, \infty}$. Definimos então um subconjunto infinito $\tilde{\mathbb{N}} \subset \mathbb{N}$ tomando como $j$-ésimo elemento de $\tilde{\mathbb{N}}$ o $j$-ésimo elemento de $\mathbb{N}_{j}$. Desta maneira, fixado $j \in \mathbb{N}$, a sequência $\left\{\Psi_{j, p_{n}}\right\}_{n \in \tilde{N}}$ é, a partir de seu $j$-ésimo termo uma subsequência de $\left\{\Psi_{j, p_{n}}\right\}_{n \in \mathbb{N}_{j}}$, logo converge para $\Psi_{j, \infty}$. Defina o subespaço $E_{j, \infty}^{c}$ como sendo o gráfico de

$$
\Psi_{j, \infty}=\lim _{\substack{n \rightarrow \infty \\ n \in \mathbb{N}}} \Psi_{j, p_{n}} .
$$

Por construção temos que $\operatorname{dim} E_{j, \infty}^{c}=d-2$. O próximo lema nos diz que os subespaços $E_{j, \infty}^{c}$ é invariante pelo cociclo de Rauzy-Veech.

Lema 6.2.5. Para todo $j \geq 0$, temos que $\Theta_{j}\left(E_{j, \infty}^{c}\right)=E_{j+1, \infty}^{c}$.

Demonstração. Denote por $n_{j} \in \mathbb{N}$ o $j$-ésimo termo de $\tilde{\mathbb{N}}$.

$$
\begin{aligned}
\Theta_{j}\left(E_{j, \infty}^{c}\right) & =\Theta_{j}\left(\lim _{\substack{n \rightarrow \infty \\
n \in \mathbb{N}}} \operatorname{graph}\left(\Psi_{j, p_{n}}\right)\right) \\
& =\lim _{\substack{n \rightarrow \infty \\
n \geq n_{j}}} \Theta_{j}\left(\operatorname{graph}\left(\Psi_{j, p_{n}}\right)\right) \\
& =\lim _{\substack{n \rightarrow \infty \\
n \geq n_{j}}} \operatorname{graph}\left(\Psi_{j+1, p_{n}}\right), \text { pelo Lema } 6.2 .2 \\
& =E_{j+1, \infty}^{c} .
\end{aligned}
$$


Agora mostraremos que o cociclo de Rauzy-Veech, é uma quase-isometria na direção central.

Proposição 6.2.3. Para todo vetor $v \in E_{0, \infty}^{c}$ e para todo $n \geq 0$, existe constante $C_{29}>1$ tal que

$$
\frac{1}{C_{29}} \cdot\|v\| \leq\left\|\Theta_{0, n} v\right\| \leq C_{29} \cdot\|v\|
$$

onde $\Theta_{0, n}=\Theta_{n} \Theta_{n-1} \cdots \Theta_{0}$.

Demonstração. Para todo $v \in E_{0, \infty}^{c}$, existe um $k \in \operatorname{Ker} \Omega_{\pi^{0}}$ tal que $v=k+\Psi_{0, \infty}(k)$. Pela continuidade do produto interno, temos que $k \perp \Psi_{0, \infty}(k)$. Logo

$$
\|k\| \leq \sqrt{\|k\|^{2}+\left\|\Psi_{0, \infty}(k)\right\|^{2}}=\|v\| \leq\|k\|+\left\|\Psi_{0, \infty}(k)\right\| \leq\|k\|+\frac{1}{\varepsilon_{0}}\|k\|,
$$

pela Proposição 6.2.2. Portanto

$$
\|k\| \leq\|v\| \leq\left(1+\frac{1}{\varepsilon_{0}}\right) \cdot\|k\| .
$$

Sabemos que ${ }^{t} \Theta_{0, n}$ : Ker $\Omega_{\pi^{n}} \rightarrow$ Ker $\Omega_{\pi^{0}}$, pelo Corolário 2.5.1, e que ${ }^{t} \Theta_{0, n}$ leva base de Ker $\Omega_{\pi^{n}}$ em base de Ker $\Omega_{\pi^{0}}$, pelo Lema 2.5.2. Seja $\mathcal{C}:=\left\{u=\left(u_{\alpha}\right)_{\alpha \in \mathcal{A}} \in \mathbb{R}^{\mathcal{A}}: u_{\alpha}=\right.$ $-1,0$, ou 1$\}$. Logo existe constante $C_{30}>0$ tal que

$$
\|\mathcal{C}\|:=\max _{u \in \mathcal{C}}\|u\| \leq C_{30} .
$$

Para todo $\pi \in \Pi^{g}$ e todo $k \in \operatorname{Ker} \Omega_{\pi}$ nós podemos escrever $k=\sum_{i=1}^{d} a_{i} u_{i}$, onde $a_{i} \in \mathbb{R}$ e $u_{i} \in \mathcal{C}(\operatorname{ver}(2.14))$. Definimos $\|k\|$ como sendo a norma da soma, ou seja, $\|k\|=\sum_{i=1}^{d}\left|a_{i}\right|$. Então

$$
\begin{aligned}
\left\|\Theta_{0, n} k\right\| & =\left\|\sum_{i=1}^{d} a_{i}{ }^{t} \Theta_{0, n} u_{i}\right\| \\
& =\left\|\sum_{i=1}^{d} a_{i} \tilde{u}_{i}\right\|, \text { onde } \tilde{u}_{i} \in \mathcal{C} \\
& \leq \sum_{i=1}^{d}\left|a_{i}\right| \cdot\left\|\tilde{u}_{i}\right\| \\
& \leq C_{30} \cdot\|k\| .
\end{aligned}
$$

Portanto

$$
\left\|^{t} \Theta_{0, n}\right\|_{\text {Ker } \Omega_{\pi^{n}}} \leq C_{30}
$$


Assim,

$$
\begin{aligned}
\left\|\Theta_{0, n} v\right\|_{\text {Ker } \Omega_{\pi^{n}}} & =\sup _{\substack{k \in \operatorname{Ker} \Omega_{\pi^{n}} \\
\|k\| \leq 1}}<\Theta_{0, n} v, k> \\
& =\sup _{\substack{k \in \operatorname{Ker} \Omega_{\pi^{n}} \\
\|k\| \leq 1}}<v,{ }^{t} \Theta_{0, n} k> \\
& \leq\|v\| \cdot\left\|^{t} \Theta_{0, n}\right\|_{\text {Ker } \Omega_{\pi^{n}}}\|k\| \\
& \leq C_{30} \cdot\|v\|, \text { por }(6.21) .
\end{aligned}
$$

Agora

$$
\begin{aligned}
\left\|\Theta_{0, n} v\right\| & \leq\left(1+\frac{1}{\varepsilon_{0}}\right)\left\|\Theta_{0, n} v\right\|_{\operatorname{Ker} \Omega_{\pi^{n}}}, \text { por }(6.20) \\
& \leq\left(1+\frac{1}{\varepsilon_{0}}\right) \cdot C_{30} \cdot\|v\| \text { por }(6.22)
\end{aligned}
$$

Agora acharemos uma estimativa inferior para $\left\|\Theta_{0, n} v\right\|$. Primeiro note que

$$
\begin{aligned}
\left\|\Theta_{0, n} v\right\|_{\text {Ker } \Omega_{\pi^{n}}} & =\sup _{\substack{k \in \operatorname{Ker} \Omega_{\pi^{n}} \\
\|k\| \leq 1}}<v,{ }^{t} \Theta_{0, n} k> \\
& \geq \sup _{\substack{\tilde{k} \in \operatorname{Ker} \Omega_{\pi^{0}} \\
\|\tilde{k}\| \leq \frac{1}{C_{1}}}}<v, \tilde{k}> \\
& =\frac{1}{C_{30}} \cdot \sup _{\substack{\tilde{k} \in \operatorname{Ker} \Omega_{\pi^{0}} \\
\|\tilde{k}\| \leq 1}}<v, \tilde{k}> \\
& =\frac{1}{C_{30}} \cdot\|v\|_{\operatorname{Ker} \Omega_{\pi^{0}}} .
\end{aligned}
$$

Usando (6.20) temos que

$$
\|v\| \leq\left(1+\frac{1}{\varepsilon_{0}}\right) \cdot\|v\|_{\text {Ker } \Omega_{\pi^{0}}} \Rightarrow\left(1+\frac{1}{\varepsilon_{0}}\right)^{-1}\|v\| \leq\|v\|_{\text {Ker } \Omega_{\pi^{0}}} .
$$

Isto junto com as estimativas (6.24) e (6.23), nos dá

$\frac{1}{C_{30}}\left(1+\frac{1}{\varepsilon_{0}}\right)^{-1}\|v\| \leq \frac{1}{C_{30}}\|v\|_{\text {Ker } \Omega_{\pi^{0}}} \leq\left\|\Theta_{0, n} v\right\|_{\text {Ker } \Omega_{\pi^{n}}} \leq\left\|\Theta_{0, n} v\right\| \leq C_{30}\left(1+\frac{1}{\varepsilon_{0}}\right)\|v\|$.

Tomando $C_{29}=C_{30}\left(1+\frac{1}{\varepsilon_{0}}\right)$ temos o resultado.

\subsection{Dinâmica do Cociclo de Rauzy-Veech}

Seja $f_{A}:[0,1) \rightarrow[0,1)$ uma t.i.i. afim sem conexão. Então $f_{A}$ é unicamente determinada pela tripla $\left(\pi,\left(\left|\tilde{I}_{\alpha}\right|\right)_{\alpha \in \mathcal{A}}, \omega^{0}\right)$, onde $\omega^{0}=\left(\omega_{\alpha}^{0}\right)_{\alpha \in \mathcal{A}} \in \mathbb{R}^{d}$ é tal que

$$
f_{A}(x)=e^{\omega_{\alpha}^{0}} x+\delta_{\alpha} \text { para todo } x \in \tilde{I}_{\alpha} .
$$


O vetor $\omega^{0}=\left(\omega_{\alpha}^{0}\right)_{\alpha \in \mathcal{A}}$ é chamado de slope vector de $f_{A}$. Denote por $\left(\omega_{\alpha}^{n}\right)_{\alpha \in \mathcal{A}}$ o slope vector de $R^{n} f_{A}$. Sabemos que $\Theta_{\pi^{n}, \varepsilon^{n}}\left(\omega^{n}\right)=\omega^{n+1}$, onde $\varepsilon^{n} \in\{0,1\}$ é o tipo de $R^{n} f_{A}$. Mais precisamente, sabemos que

$$
\begin{aligned}
\omega_{\alpha}^{n+1} & =\omega_{\alpha}^{n} \text { if } \alpha \neq \alpha^{n}(1-\varepsilon) \\
\omega_{\alpha^{n}(1-\varepsilon)}^{n+1} & =\omega_{\alpha^{n}(\varepsilon)}^{n}+\omega_{\alpha^{n}(1-\varepsilon)}^{n},
\end{aligned}
$$

onde $\alpha^{n}(\varepsilon)$ e $\alpha^{n}(1-\varepsilon)$ são as letras vencedoras e perdedoras de $R^{n}\left(f_{\mathcal{A}}\right)$, respectivamente. Portanto

$$
\Theta_{n}\left(\omega^{n}\right):=\Theta_{\pi^{n}, \varepsilon^{n} n}\left(\omega^{n}\right)=\omega^{n+1},
$$

onde $\Theta_{n}$ foi definido em (2.6). Repetindo-se este processo indutivamente nós temos

$$
\Theta_{n} \Theta_{n-1} \cdots \Theta_{1} \Theta_{0}\left(\omega^{0}\right)=\omega^{n} .
$$

Seja $f \in \mathcal{B}_{k}^{2+\nu}$ tal que $\int_{I} D^{2} f(s) / D f(s) d s=0$. Defina o vetor $L_{n}=\left(L_{n, \alpha}\right)_{\alpha \in \mathcal{A}}$ por

$$
L_{n, \alpha}=\frac{1}{\left|I_{\alpha}^{n}\right|} \int_{I_{\alpha}^{n}} \ln D f_{n}(s) d s=\frac{1}{\left|I_{\alpha}^{n}\right|} \int_{I_{\alpha}^{n}} \ln D f^{q_{n}^{\alpha}}(s) d s .
$$

Note que se $f$ é uma t.i.i. afim então $L_{n, \alpha}=\omega_{\alpha}^{n}$ para todo $\alpha \in \mathcal{A}$ e para todo $n \geq 0$. A próxima proposição nos diz que a sequência de vetores $L_{n}$ é uma pseudo-órbita para o cociclo de Rauzy-Veech.

Proposição 6.3.1. Let $f \in \mathcal{B}_{k}^{2+\nu} \operatorname{com} \int_{I} D^{2} f(s) / D f(s) d s=0$. Então para todo $n \geq 0$

$$
L_{n+1}=\Theta_{n} L_{n}+\overrightarrow{\epsilon_{n}}
$$

onde $\left\|\overrightarrow{\epsilon_{n}}\right\|=\mathrm{O}\left(\lambda_{5}^{\sqrt{n}}\right)$.

Demonstração. Denote por $x_{\alpha}^{n} \in I_{\alpha}^{n}$ o ponto tal que $L_{n, \alpha}=\ln D f_{\alpha}^{n}\left(x_{\alpha}^{n}\right)$, para todo $n \geq 0$.

- Se $\alpha \neq \alpha^{n}(\varepsilon), \alpha^{n}(1-\varepsilon)$ então claramente $L_{n+1, \alpha}=L_{n, \alpha}$.

- Se $\alpha=\alpha^{n}(\varepsilon)$ então $q_{\alpha^{n}(\varepsilon)}^{n}=q_{\alpha^{n}(\varepsilon)}^{n+1}$ e portanto

$$
\begin{aligned}
L_{n+1, \alpha} & =\ln D f^{q_{\alpha}^{n}}\left(x_{\alpha}^{n+1}\right) \\
& =\ln D f^{q_{\alpha}^{n}}\left(x_{\alpha}^{n+1}\right)+\ln D f^{q_{\alpha}^{n}}\left(x_{\alpha}^{n}\right)-\ln D f^{q_{\alpha}^{n}}\left(x_{\alpha}^{n}\right) \\
& =L_{n, \alpha}+\ln D f_{\alpha}^{q_{\alpha}^{n}}\left(x_{\alpha}^{n+1}\right)-\ln D f^{q_{\alpha}^{n}}\left(x_{\alpha}^{n}\right) \\
& =L_{n, \alpha}+\mathrm{O}\left(\lambda_{5}^{\sqrt{n}}\right),
\end{aligned}
$$

by Proposition 5.2.1. 
- Se $\alpha=\alpha^{n}(1-\varepsilon)$ então $q_{\alpha^{n}(1-\varepsilon)}^{n+1}=q_{\alpha^{n}(1-\varepsilon)}^{n}+q_{\alpha^{n} n(\varepsilon)}^{n}$. Note também que $f^{q_{\alpha(1-\varepsilon)}^{n}}\left(x_{\alpha^{n} n(1-\varepsilon)}^{n}\right) \in I_{\alpha^{n}(\varepsilon)}^{n}$. Portanto

$$
\begin{aligned}
& L^{n+1, \alpha^{n}(1-\varepsilon)}=\ln D f^{q_{\alpha^{n}(1-\varepsilon)}^{n+1}}\left(x_{\alpha^{n}(1-\varepsilon)}^{n+1}\right) \\
& =\ln D f^{q_{\alpha^{n}(\varepsilon)}^{n}}\left(f^{q_{\alpha^{n}(1-\varepsilon)}^{n}}\left(x_{\alpha^{n}(1-\varepsilon)}^{n+1}\right)\right)+\ln D f^{q_{\alpha^{n}(1-\varepsilon)}^{n}}\left(x_{\alpha^{n}(1-\varepsilon)}^{n+1}\right) \\
& =\ln D f^{q_{\alpha^{n}(\varepsilon)}^{n}}\left(f^{q_{\alpha^{n}(1-\varepsilon)}^{n}}\left(x_{\alpha^{n}(1-\varepsilon)}^{n+1}\right)\right)-\ln D f^{q_{\alpha^{n}(\varepsilon)}^{n}}\left(x_{\alpha^{n}(\varepsilon)}^{n}\right)+L_{\alpha^{n}(\varepsilon)}^{n} \\
& +\ln D f^{q_{\alpha^{n}(1-\varepsilon)}^{n}}\left(x_{\alpha^{n}(1-\varepsilon)}^{n+1}\right)-\ln D f_{\alpha^{n}(1-\varepsilon)}^{q^{n}}\left(x_{\alpha^{n}(1-\varepsilon)}^{n}\right)+L_{\alpha^{n}(1-\varepsilon)}^{n} \\
& =L_{n, \alpha^{n}(\varepsilon)}+L_{n, \alpha^{n}(1-\varepsilon)}+\mathrm{O}\left(\lambda_{5}^{\sqrt{n}}\right) \text {, }
\end{aligned}
$$

pela Proposição 5.2.1.

Isto termina a prova.

Seja $L_{n}$ o vetor dado pela definição (6.27). Para todo $n \in \mathbb{N}$ usaremos a seguinte decomposição

$$
L_{n}=L_{n}^{s} \oplus L_{n}^{c} \oplus L_{n}^{u} \in E_{n}^{s} \oplus E_{n, \infty}^{c} \oplus E_{n}^{u}
$$

Lema 6.3.1. A sequência $\left\{L_{n}^{s}\right\}_{n \in \mathbb{N}}$ converge para o vetor nulo.

Demonstração. Sabemos pela Proposição 6.1.1 que para todo $v \in E_{j}^{s}$ temos que para todo $n \geq 0$

$$
\left\|\Theta_{j+n-1} \cdot \Theta_{j+n-2} \cdots \Theta_{j} v\right\| \leq \frac{1}{C_{s} \cdot \mu^{n}}\|v\|
$$

Substituindo a norma acima, por uma equivalente, que chamamos de norma adaptada (ver [Shu87, Proposição 4.2]) e, ainda denotaremos por $\|\cdot\|$, por simplicidade, podemos encontrar uma constante $\mu>\tilde{\mu}>1$ tal que para todo $n \geq 0$ e para todo $v \in E_{n}^{s}$ temos

$$
\left\|\Theta_{n} v\right\| \leq \frac{1}{\tilde{\mu}}\|v\|
$$

Pela Proposição 6.3.1 sabemos que para todo $n \geq 0$

$$
L_{n}^{s}=\Theta_{n-1} L_{n-1}^{s}+\varepsilon_{n-1}^{s},
$$

e portanto $\left\|L_{n}^{s}\right\| \leq \frac{1}{\tilde{\mu}}\left\|L_{n-1}^{s}\right\|+C \cdot \lambda_{5}^{\sqrt{n-1}}$. Aplicando estas estimativas $n$ vezes temos que

$$
\left\|L_{n}^{s}\right\| \leq \frac{1}{\tilde{\mu}^{n}} \cdot\left\|L_{0}^{s}\right\|+C \cdot \sum_{i=0}^{n-1} \frac{1}{\tilde{\mu}^{i}} \lambda_{5}^{\sqrt{n-i-1}} .
$$

Sabemos que a sequência $\left\{L_{n}^{s}\right\}_{n \in \mathbb{N}}$ é uniformemente limitada, logo

$$
\frac{1}{\tilde{\mu}^{n}} \cdot\left\|L_{0}^{s}\right\| \rightarrow 0 \text { quando } n \rightarrow \infty
$$


Analisaremos agora a outra parcela. Denote por $a_{n, i}=\frac{1}{\tilde{\mu}^{i}} \cdot \lambda_{5}^{\sqrt{n-i+1}}$. Então

$$
\frac{a_{n, i+1}}{a_{n, i}}=\frac{1}{\tilde{\mu}} \cdot \lambda_{5}^{\sqrt{n-i-2}-\sqrt{n-i-1}} \leq \frac{1}{\tilde{\mu}} \cdot \lambda_{5}^{\frac{-1}{2 \sqrt{n-i-2}}}
$$

onde a desigualdade acima é dada pelo Teorema do valor médio. Seja $n_{0} \in \mathbb{N}$ tal que se $n-i-2 \geq n_{0}$ então

$$
\lambda_{5}^{\frac{-1}{2 \sqrt{n-i-2}}} \leq \frac{1}{\tilde{\mu}}
$$

Assim

$$
\sum_{i=0}^{n-1} \frac{1}{\tilde{\mu}^{i}} \lambda_{5}^{\sqrt{n-i-1}}=\sum_{i=0}^{n-n_{0}-2} \frac{1}{\tilde{\mu}^{i}} \lambda_{5}^{\sqrt{n-i-1}}+\sum_{i=n-n_{0}-1}^{n-1} \frac{1}{\tilde{\mu}^{i}} \lambda_{5}^{\sqrt{n-i-1}} .
$$

Analisando cada soma acima, em separado, obtemos

$$
\begin{aligned}
\sum_{i=0}^{n-n_{0}-2} \frac{1}{\tilde{\mu}^{i}} \lambda_{5}^{\sqrt{n-i-1}} & =\sum_{i=0}^{n-n_{0}-2} a_{n, i} \\
& \leq \sum_{i=0}^{n-n_{0}-2} \frac{1}{\tilde{\mu}^{2 i}} \lambda_{5}^{\sqrt{n}} \\
& \leq \lambda_{5}^{\sqrt{n}} \cdot \sum_{i=0}^{n-n_{0}-2} \frac{1}{\tilde{\mu}^{2 i}}, \text { por }(6.30) \\
& \leq \frac{1}{1-\tilde{\mu}^{-2}} \cdot \lambda_{5}^{\sqrt{n}}
\end{aligned}
$$

$\mathrm{e}$

$$
\begin{aligned}
\sum_{i=n-n_{0}-1}^{n-1} \frac{1}{\tilde{\mu}^{i}} \lambda_{5}^{\sqrt{n-i-1}} & \leq \sum_{i=n-n_{0}-1}^{n-1} \frac{1}{\tilde{\mu}^{i}} \\
& \leq \frac{1}{\tilde{\mu}^{n}} \sum_{i=n-n_{0}-1}^{n-1} \frac{1}{\tilde{\mu}^{i-n}} \\
& \leq C \cdot \frac{1}{\tilde{\mu}^{n}}
\end{aligned}
$$

Das equações (6.29), (6.31) e (6.32), obtemos o resultado.

Lema 6.3.2. A sequência $\left\{L_{n}^{u}\right\}_{n \in \mathbb{N}}$ converge para o vetor nulo.

Demonstração. A prova é similar ao do Lema 6.3.1 e novamente usamos uma norma adaptada. Para todo $n \leq 0$ temos que

$$
\left\|L_{n+1}^{u}\right\| \geq \tilde{\mu} \cdot\left\|L_{n}^{u}\right\|-C \cdot \lambda_{5}^{\sqrt{n}} .
$$


Aplicando essa estimativa $k$ vezes, obtemos

$$
\left\|L_{n+k}^{u}\right\| \geq \tilde{\mu}^{k}\left\|L_{n}^{u}\right\|-C \cdot \sum_{j=0}^{k-1} \tilde{\mu}^{j} \cdot \lambda_{5}^{\sqrt{n+k-1-j}},
$$

e portanto

$$
\left\|L_{n}^{u}\right\| \leq \frac{1}{\tilde{\mu}^{k}}\left\|L_{n+k}^{u}\right\|+C \cdot \sum_{j=0}^{k-1} \tilde{\mu}^{j-k} \cdot \lambda_{5}^{\sqrt{n+k-1-j}} .
$$

Fazendo $k=n$, temos

$$
\left\|L_{n}^{u}\right\| \leq \frac{1}{\tilde{\mu}^{n}}\left\|L_{2 n}^{u}\right\|+C \cdot \sum_{j=0}^{n-1} \tilde{\mu}^{j-n} \cdot \lambda_{5}^{\sqrt{2 n-1-j}} .
$$

A sequência $\left\{L_{2 n}^{u}\right\}_{n \in \mathbb{N}}$ é uniformemente limitada, e isto nos dá

$$
\frac{1}{\tilde{\mu}^{n}}\left\|L_{2 n}^{u}\right\| \rightarrow 0, \text { quando } n \rightarrow \infty
$$

Agora, analisaremos o somatório acima. Primeiro note que

$$
\sum_{j=0}^{n-1} \tilde{\mu}^{j-n} \cdot \lambda_{5}^{\sqrt{2 n-1-j}}=\sum_{s=0}^{n-1} \tilde{\mu}^{-1-s} \cdot \lambda_{5}^{\sqrt{n+s}} .
$$

Seja $a_{s}=\tilde{\mu}^{-1-s} \cdot \lambda_{5}^{\sqrt{n+s}}$. Então

$$
\frac{a_{s+1}}{a_{s}}=\frac{1}{\tilde{\mu}} \cdot \lambda_{5}^{\sqrt{n+s+1}-\sqrt{n+s}} \leq \frac{1}{\tilde{\mu}} \cdot \lambda_{5}^{\frac{1}{2 \sqrt{n+s+1}}}
$$

Logo

$$
\begin{aligned}
\sum_{s=0}^{n-1} \tilde{\mu}^{-1-s} \cdot \lambda_{5}^{\sqrt{n+s}} & =\tilde{\mu}^{-1} \lambda_{5}^{\sqrt{n}}+\tilde{\mu}^{-2} \lambda_{5}^{\sqrt{n+1}}+\cdots+\tilde{\mu}^{-n} \lambda_{5}^{\sqrt{2 n-1}} \\
& \leq \tilde{\mu}^{-1} \lambda_{5}^{\sqrt{n}}\left(1+\tilde{\mu}^{-1} \lambda_{5}^{\sqrt{n+1}-\sqrt{n}}+\tilde{\mu}^{-2} \lambda_{5}^{\sqrt{n+2}-\sqrt{n}}+\cdots+\tilde{\mu}^{-n+1} \lambda_{5}^{\sqrt{2 n-1}-\sqrt{n}}\right) \\
& \leq \tilde{\mu}^{-1} \lambda_{5}^{\sqrt{n}}\left(1+\tilde{\mu}^{-1} \lambda_{5}^{\frac{1}{2 \sqrt{n+1}}}+\ldots+\tilde{\mu}^{-n+1} \lambda_{5}^{\frac{1}{2 \sqrt{n+1}}+\ldots+\frac{1}{2 \sqrt{2 n-1}}}\right) \quad \text { por }(6.34) \\
& \leq \tilde{\mu}^{-1} \lambda_{5}^{\sqrt{n}}\left(1+\tilde{\mu}^{-1}+\ldots+\tilde{\mu}^{-n+1}\right) \\
& \leq \tilde{\mu}^{-1} \lambda_{5}^{\sqrt{n}} \cdot \frac{1}{1-\tilde{\mu}^{-1}} .
\end{aligned}
$$

De (6.33) e (6.35) temos o resultado. 
Defina para todo $n \geq 0$ o seguinte vetor:

$$
\tilde{\omega}_{n}:=\Theta_{0}^{-1} \Theta_{1}^{-1} \cdots \Theta_{n-1}^{-1}\left(L_{n}^{c}\right) .
$$

Lema 6.3.3. A sequência $\left\{\tilde{\omega}_{n}\right\}_{n \in \mathbb{N}}$ converge.

Demonstração. Mostraremos que $\left\{\tilde{\omega}_{n}\right\}_{n \in \mathbb{N}}$ é uma sequência de Cauchy. Pela Proposição 6.3.1 sabemos que

$$
L_{n+1}^{c}=\Theta_{n} L_{n}^{c}+\varepsilon_{n}^{c}
$$

Então

$$
\begin{aligned}
\tilde{\omega}_{n+1} & =\Theta_{0}^{-1} \cdots \Theta_{n}^{-1} L_{n+1}^{c} \\
& =\Theta_{0}^{-1} \cdots \Theta_{n-1}^{-1} L_{n}^{c}+\Theta_{0}^{-1} \cdots \Theta_{n}^{-1} \Theta_{0}^{-1} \cdots \Theta_{n}^{-1} \varepsilon_{n} \\
& =\tilde{\omega}_{n}+\tilde{\varepsilon}_{n},
\end{aligned}
$$

onde $\tilde{\varepsilon}_{n}=\mathrm{O}\left(\lambda_{5}^{\sqrt{n}}\right)$, pela Proposição 6.2.3.

Lema 6.3.4. Seja $\omega=\lim _{n \rightarrow \infty} \tilde{\omega}_{n} \in E_{0, \infty}^{c}$ e $\omega^{n} \in E_{n, \infty}^{c}$ a órbita pelo cociclo de RauzyVeech de $f$, ou seja, $\omega^{n}=\Theta_{0} \cdots \Theta_{n-1} \omega$. Então

$$
\left\|\omega^{n}-L_{n}\right\|=\mathrm{O}\left(\lambda_{5}^{\sqrt{n}}\right)
$$

Demonstração. Pelos Lemas 6.3.1 e 6.3.2 basta estimarmos $\omega^{n}-L_{n}^{c}$.

$$
\begin{aligned}
\left\|\omega^{n}-L_{n}^{c}\right\| & =\left\|\Theta_{0} \cdots \Theta_{n-1} \omega-L_{n}^{c}\right\| \\
& \leq\left\|\left.\Theta_{0} \cdots \Theta_{n-1}\right|_{E_{0 . \infty}^{c}}\right\| \cdot\left\|\omega-\left(\Theta_{0} \cdots \Theta_{n-1}\right)^{-1} L_{n}^{c}\right\| \\
& \leq C_{30} \cdot\left(1+\frac{1}{\varepsilon_{0}}\right) \cdot\left\|\omega-\tilde{\omega}_{n}\right\|, \text { pela Proposição } 6.2 .3 \\
& =\mathrm{O}\left(\lambda_{5}^{\sqrt{n}}\right), \text { pelo Lema } 6.3 .3 .
\end{aligned}
$$

Seja Aff $\left(\left\{\pi^{i}, \varepsilon^{i}\right\}_{i \in \mathbb{N}}, \omega\right)$ o conjunto das t.i.i. afins cuja a combinatória é $\left\{\pi^{i}, \varepsilon^{i}\right\}_{i \in \mathbb{N}}$ e cujo slope vector é $\omega$. Denote por $\operatorname{Aff}^{(1)}\left(\left\{\pi^{i}, \varepsilon^{i}\right\}_{i \in \mathbb{N}}, \omega\right)$ o conjunto das t.i.i. afins em $\operatorname{Aff}\left(\left\{\pi^{i}, \varepsilon^{i}\right\}_{i \in \mathbb{N}}, \omega\right)$ cujo o domínio é o intervalo $[0,1]$. O próximo resultado nos dá uma condição necessária e suficiente para que $\operatorname{Aff}(\gamma, \omega) \neq \emptyset$. Este resultado foi estabelecido em [MMY09, Proposição 2.3].

Proposição 6.3.2. O conjunto $\operatorname{Aff}\left(\left\{\pi^{i}, \varepsilon^{i}\right\}_{i \in \mathbb{N}}, \omega\right)$ é não-vazio, se e somente se, o hiperplano $\left\{\sum_{\alpha \in \mathcal{A}} \chi_{\alpha} \omega_{\alpha}=0\right\}$ contém uma t.i.i. standard cuja a combinatória é igual $a\left\{\pi^{i}, \varepsilon^{i}\right\}_{i \in \mathbb{N}}$. Neste caso, o conjunto $\operatorname{Aff}^{(1)}\left(\left\{\pi^{i}, \varepsilon^{i}\right\}_{i \in \mathbb{N}}, \omega\right)$, é convexo e compacto. 
Quando existe uma única (a menos de reescalonamento) t.i.i. standard cuja combinatória é $\left\{\pi^{i}, \varepsilon^{i}\right\}_{i \in \mathbb{N}}$, a condição descrita na Proposição 6.3.2 significa que o vetor $\omega$ pertence ao hiperplano $\left\{\sum_{\alpha \in \mathcal{A}} \chi_{\alpha} \omega_{\alpha}=0\right\}$.

Em geral, como mencionado acima, $\operatorname{Aff}\left(\left\{\pi^{i}, \varepsilon^{i}\right\}_{i \in \mathbb{N}}, 0\right)$ é um cone simplicial de dimensão $r \leq g$, onde $g$ é o genus da superfície associada a classe de Rauzy de $\left\{\pi^{i}, \varepsilon^{i}\right\}_{i \in \mathbb{N}}$. Denote por $\chi^{(1)}, \ldots, \chi^{(r)}$ os vetores extremais normalizados deste cone simplicial. Uma condição necessária e suficiente para assegurar que $\operatorname{Aff}\left(\left\{\pi^{i}, \varepsilon^{i}\right\}_{i \in \mathbb{N}}, \omega\right)$ é não-vazio é que os números

$$
\sum_{\alpha \in \mathcal{A}} \chi_{\alpha}^{(j)} \omega_{\alpha}, j=1, \ldots, r
$$

não são todos estritamentes positivos, nem todos estritamentes negativos. (ver [MMY09].)

Em nosso caso, $g=1$, temos que $\operatorname{Aff}^{(1)}\left(\left\{\pi^{i}, \varepsilon^{i}\right\}_{i \in \mathbb{N}}, \omega\right)$ é unitário. Este elemento nós chamamos de modelo afim de $f$ e o denotamos por $f_{A}$.

\subsection{Demonstração do Teorema E}

É claro que, por construção, a t.i.i. afim $f_{A}$ construída na seção anterior satisfaz a condição $(i)$. Suponhamos, sem perda de generalidade, que $f \in \mathcal{B}_{k}^{2+\nu}$ possua apenas uma descontinuidade. Para demonstrar (ii) precisaremos do seguinte lema:

Proposição 6.4.1. Sejam $f \in \mathcal{B}_{k, *}^{2+\nu}$ tal que $f$ só possua um único ponto de descontinuidade, digamos $\partial I_{\alpha^{*}}$ e $f_{A}$ seu modelo afim. Então $B P_{f}\left(\partial I_{\alpha}\right)=B P_{f_{A}}\left(\partial \tilde{I}_{\alpha}\right)$ para todo $\alpha \in \mathcal{A}$ tal que $\alpha \neq \alpha^{*}$ e $\pi_{0}(\alpha)>1$.

Demonstração. Sabemos pelo Corolário 2.6.2 que existe subsequência $n_{1}<n_{2}<$ $\ldots<n_{s}<\ldots$ tal que $R_{\text {rot }}^{s} f=R^{n_{s}} f$. Para todo $s>0$ seja $\partial I_{\alpha_{s}^{*}}^{n_{s}}$ o único ponto de descontinuidade de $R^{n_{s}} f$. Então para todo $\gamma \in \mathcal{A} \operatorname{com} \pi_{0}^{n_{s}}(\gamma)>1$ e $\gamma \neq \alpha_{s}^{*}$ temos que existe um único $1 \leq j_{\gamma}<q_{\gamma}^{n_{s}}$ e um único $\alpha \in \mathcal{A}$ tal que $f^{j_{\gamma}}\left(\partial I_{\gamma}^{n_{s}}\right)=\partial I_{\alpha}$, pois $f$ não tem conexão.

Afirmamos que $\alpha \neq \alpha^{*}$ e que $\pi_{0}(\alpha)>1$. De fato, suponhamos que $f^{j_{\gamma}}\left(\partial I_{\gamma}^{n_{s}}\right)=\partial I_{\alpha^{*}}$. Então $f^{j_{\gamma}+1}\left(\partial I_{\gamma}^{n_{s}}\right)=0=f^{q_{\alpha^{*}}^{n_{s}}}\left(\partial I_{\alpha_{s}^{*}}^{n_{s}}\right)$. Suponhamos que $q_{\gamma}^{n_{s}} \leq q_{\alpha_{s}^{*}}^{n_{s}}$, o outro caso é analisado similarmente. Então $f^{q_{\alpha_{s}^{*}}^{n_{s}-j_{\gamma}-1}}\left(\partial I_{\alpha_{s}^{*}}^{n_{s}}\right)=\partial I_{\gamma}^{n_{s}} \in I^{n_{s}}$. Como $q_{\alpha_{s}^{*}}^{n_{s}}$ é o tempo de primeiro retorno de $I_{\alpha_{s}^{*}}^{n_{s}}$ por $f$ ao intervalo $I^{n_{s}}$, temos que $q_{\alpha_{s}^{*}}^{n_{s}}-j_{\gamma}-1=q_{\alpha_{s}^{*}}^{n_{s}}$, o que é absurdo. Agora se $\pi_{0}(\alpha)=1$ temos que $\partial I_{\alpha}=0$, logo $f^{j_{\gamma}}\left(\partial I_{\gamma}^{n_{s}}\right)=0=f^{q_{\alpha^{*}}}\left(\partial I_{\alpha_{s}^{*}}^{n_{s}}\right)$, ou seja, $f_{\alpha_{s}^{*}}^{n_{s}^{n_{s}}-j_{\gamma}}\left(\partial I_{\alpha_{s}^{*}}^{n_{s}}\right)=\partial I_{\gamma}^{n_{s}} \in I^{n_{s}}$, o que pelo mesmo argumento anterior nos dá um absurdo. 
Agora pela definição de $n_{s}$ e de $R_{\text {rot }}$ temos que $q_{\beta}^{n_{s}}=q_{\gamma}^{n_{s}}$, onde $\beta \in \mathcal{A}$ é tal que $\pi_{0}^{n_{s}}(\beta)+1=\pi_{0}^{n_{s}}(\gamma)$. Então

$$
\begin{aligned}
B P_{R^{n_{s}}}\left(\partial I_{\gamma}^{n_{s}}\right) & =\ln D_{-} R^{n_{s}} f\left(\partial I_{\gamma}^{n_{s}}\right)-\ln D_{+} R^{n_{s}} f\left(\partial I_{\gamma}^{n_{s}}\right) \\
& =\sum_{i=0}^{q_{\beta}^{n_{s}}-1} \ln D_{-} f\left(f^{i}\left(\partial I_{\gamma}^{n_{s}}\right)\right)-\sum_{i=0}^{q_{\gamma}^{n_{s}}-1} \ln D_{+} f\left(f^{i}\left(\partial I_{\gamma}^{n_{s}}\right)\right) \\
& =\sum_{i=0}^{q_{\gamma}^{n_{s}}-1}\left(\ln D_{-} f\left(f^{i}\left(\partial I_{\gamma}^{n_{s}}\right)\right)-\ln D_{+} f\left(f^{i}\left(\partial I_{\gamma}^{n_{s}}\right)\right)\right) \\
& =\ln D_{-} f\left(f^{j_{\gamma}}\left(\partial I_{\gamma}^{n_{s}}\right)\right)-\ln D_{+} f\left(f^{j_{\gamma}}\left(\partial I_{\gamma}^{n_{s}}\right)\right) \\
& =\ln D_{-} f\left(\partial I_{\alpha}\right)-\ln D_{+} f\left(\partial I_{\alpha}\right) \\
& =B P_{f}\left(\partial I_{\alpha}\right)
\end{aligned}
$$

Como $f$ e seu modelo afim $f_{A}$ possuem a mesma combinatória temos que

$$
B P_{R^{n_{s} f_{A}}}\left(\partial \tilde{I}_{\gamma}^{n_{s}}\right)=B P_{f_{A}}\left(\partial \tilde{I}_{\alpha}\right)
$$

Portanto,

$$
\begin{aligned}
B P_{f}\left(\partial I_{\alpha}\right)-B P_{f_{A}}\left(\partial \tilde{I}_{\alpha}\right)= & B P_{R^{n_{s} f}}\left(\partial I_{\gamma}^{n_{s}}\right)-B P_{R^{n_{s} f_{A}}}\left(\partial \tilde{I}_{\gamma}^{n_{s}}\right) \\
= & \ln D_{-} R^{n_{s}} f\left(\partial I_{\gamma}^{n_{s}}\right)-\ln D_{+} R^{n_{s}} f\left(\partial I_{\gamma}^{n_{s}}\right) \\
& -\ln D_{-} R^{n_{s}} f_{A}\left(\partial \tilde{I}_{\gamma}^{n_{s}}\right)+\ln D_{+} R^{n_{s}} f_{A}\left(\partial \tilde{I}_{\gamma}^{n_{s}}\right) \\
= & L_{n_{s}, \beta}-L_{n_{s}, \gamma}+\mathrm{O}\left(\lambda^{\sqrt{n_{s}}}\right)-\omega_{\beta}^{n_{s}}+\omega_{\gamma}^{n_{s}} \\
= & \mathrm{O}\left(\lambda^{\sqrt{n_{s}}}\right), \text { pela Proposição 6.3.4. }
\end{aligned}
$$

Fazendo $s \rightarrow \infty$ temos que $B P_{f}\left(\partial I_{\alpha}\right)=B P_{f_{A}}\left(\partial \tilde{I}_{\alpha}\right)$.

A Proposição 6.4.1 nos mostra que $f$ e $f_{A}$ possuem $d-2$ breaks iguais.

Lema 6.4.1. Sejam $f, g \in \mathcal{B}^{2+k}$ break-equivalentes, com combinatórias iguais e apenas uma descontinuidade. Então os modelos afim de $f$ e $g$ são iguais, ou seja, $f_{A}=g_{A}$.

Demonstração. Por simplicidade denote $\mathcal{A}=\{1,2, \ldots, d\}$ e $\left\{I_{j}\right\}_{j \in \mathcal{A}},\left\{J_{j}\right\}_{j \in \mathcal{A}}$ as partições de $f_{A}$ e $g_{A}$ respectivamente. Seja $j_{0} \in \mathcal{A}$ tal que $\partial I_{j_{0}}$ e $\partial J_{j_{0}}$ são os pontos de descontinuidade de $f$ e $g$ respectivamente. Como $f$ e $g$ são break-equivalentes, temos pela Proposição 6.4.1 que

$$
\omega_{i+1}^{f}-\omega_{i}^{f}=\omega_{i+1}^{g}-\omega_{i}^{g} \text { para todo } i \in \mathcal{A} \text { tal que } i \neq j_{0}-1, d,
$$


o que é equivalente a

$$
\begin{gathered}
\omega_{1}^{f}-\omega_{1}^{g}=\ldots=\omega_{j_{0}-1}^{f}-\omega_{j_{0}-1}^{g}, \\
\omega_{j_{0}}^{f}-\omega_{j_{0}}^{g}=\ldots=\omega_{d}^{f}-\omega_{d}^{g},
\end{gathered}
$$

onde $\omega^{f}=\left(\omega_{i}^{f}\right)_{i \in \mathcal{A}} \in E_{0, \infty}^{c}$ e $\omega^{g}=\left(\omega_{i}^{g}\right)_{i \in \mathcal{A}} \in E_{0, \infty}^{c}$ são os slope-vector de $f_{A}$ e $g_{A}$ respectivamente, obtidos pelo Lema 6.3.4. Denotando por $v:=\omega_{1}^{f}-\omega_{1}^{g}$ e por $\tilde{v}:=\omega_{j_{0}}^{f}-\omega_{j_{0}}^{g}$ temos que

$$
\omega^{f}-\omega^{g}=\left(\omega_{i}^{f}-\omega_{i}^{g}\right)_{i \in \mathcal{A}}=(v, \ldots, v, \underbrace{\tilde{v}}_{j_{0}-\text { posição }}, \ldots, \tilde{v}) \in E_{0, \infty}^{c},
$$

ou seja o vetor $\omega^{f}-\omega^{g}$ pode ser visto como um slope-vector de uma t.i.i. afim de dois intervalos. Pela Proposição 2.6.1 temos que a renormalização da t.i.i. afim com slope-vector $(v, \tilde{v})$ é uma subsequência da renormalização da afim de $d$ intervalos com slope-vector $\omega^{f}-\omega^{g}$. Assim $(v, \tilde{v}) \in E_{0, \infty}^{c}(2)$, onde $E_{0, \infty}^{c}(2)$ é o espaço central definido pela renormalização de dois intervalos. Como $\operatorname{dim} E_{0, \infty}^{c}(2)=0$ temos que $v=\tilde{v}=0$ e portanto $\omega^{f}=\omega^{g}$.

Para demonstrar (iii) primeiro note que como $R^{n} f_{A}$ é uma t.i.i. afim então $Z_{\tilde{I}_{\alpha}^{n}} R^{n} f_{A}=\mathrm{Id}$ para todo $n \geq 0$ e para todo $\alpha \in \mathcal{A}$. Então por (5.5) temos que

$$
\left\|Z_{I_{\alpha}^{n}} R^{n} f-Z_{\tilde{I}_{\alpha}^{n}} R^{n} f_{A}\right\|_{C^{2}}=\mathrm{O}\left(\lambda_{5}^{\sqrt{n}}\right)
$$

Resta estimarmos $\left\|\left(I_{\alpha}^{n}\right)_{\alpha \in \mathcal{A}}-\left(\tilde{I}_{\alpha}^{n}\right)_{\alpha \in \mathcal{A}}\right\|_{1}$ e $\left\|\left(R^{n} f\left(I_{\alpha}\right)\right)_{\alpha \in \mathcal{A}}-\left(R^{n} f_{A}\left(\tilde{I}_{\alpha}\right)\right)_{\alpha \in \mathcal{A}}\right\|_{1}$. Faremos isto na próxima seção.

\subsubsection{Métrica Projetiva}

O início desta seção segue de [Via08, Seção 4.6]. Considere o cone convexo $\mathbb{R}_{+}^{A}$. Dado qualquer $\theta, \gamma \in \mathbb{R}_{+}^{A}$, defina

$$
a(\theta, \gamma)=\inf _{\alpha \in \mathcal{A}} \frac{\theta_{\alpha}}{\gamma_{\alpha}} \quad \text { e } \quad b(\theta, \gamma)=\sup _{\beta \in \mathcal{A}} \frac{\theta_{\beta}}{\gamma_{\beta}}
$$

A métrica projectiva associado a $\mathbb{R}_{+}^{A}$ é definida por

$$
\mathrm{d}_{p}(\lambda, \gamma)=\log \frac{b(\theta, \gamma)}{a(\theta, \gamma)}=\log \sup _{\theta, \beta \in \mathcal{A}} \frac{\theta_{\alpha} \gamma_{\beta}}{\gamma_{\alpha} \theta_{\beta}}
$$

Segue da definição de $\mathrm{d}_{p}$ que para todo $\theta, \gamma, \zeta \in \mathbb{R}_{+}^{A}$ 
(a) $\mathrm{d}_{p}(\theta, \gamma)=\mathrm{d}_{p}(\gamma, \theta)$;

(b) $\mathrm{d}_{p}(\theta, \gamma)=\mathrm{d}_{p}(\theta, \zeta)+\mathrm{d}_{p}(\zeta, \gamma)$;

(c) $\mathrm{d}_{p}(\theta, \gamma) \geq 0$

(d) $\mathrm{d}_{p}(\theta, \gamma)=0$ se e somente se existe $t>0$ tal que $\theta=t \gamma$.

Seja $S: \mathbb{R}^{\mathcal{A}} \rightarrow \mathbb{R}^{\mathcal{A}}$ um operador linear tal que $S\left(\mathbb{R}_{+}^{A}\right) \subset \mathbb{R}_{+}^{A}$ ou, equivalentemente, $S_{\alpha \beta} \geq 0$ para todo $\alpha, \beta \in \mathcal{A}$, onde $S_{\alpha \beta}$ são as entradas da matriz $S$. Então para todo $\alpha, \gamma \in \mathbb{R}^{\mathcal{A}}$,

$$
\mathrm{d}_{p}(S(\theta), S(\gamma)) \leq \mathrm{d}_{p}(\theta, \gamma)
$$

Now, we define $s: \Delta_{\mathcal{A}} \rightarrow \Delta_{\mathcal{A}}$ by

$$
s(\theta)=\frac{S(\theta)}{\sum_{\alpha \in \mathcal{A}} S(\theta)_{\alpha}}=\frac{S(\theta)}{\sum_{\alpha, \beta \in \mathcal{A}} S_{\alpha \beta} \theta_{\beta}} .
$$

Nós dizemos $s$ é a projectivização de $S$.

A próxima proposição, cuja a prova pode ser encontrada em [Via08, Proposição 4.26], assegura que se $s\left(\Delta_{\mathcal{A}}\right)$ tem diâmetro finito na métrica $\mathrm{d}_{p}-$ em $\Delta_{\mathcal{A}}$ então $g$ é uma contração uniforme relativa a métrica projectiva:

Proposição 6.4.2. Para qualquer $\Delta>0$ existe $\kappa<1$ tal que se o diâmetro de $G\left(\mathbb{R}_{+}^{\mathcal{A}}\right)$ relativo a $\mathrm{d}_{p}$ é menor que $\Delta$ então

$$
\mathrm{d}_{p}(S(\theta), S(\gamma)) \leq \kappa \cdot \mathrm{d}_{p}(\theta, \gamma) \quad \text { for all } \quad \lambda, \gamma \in \mathbb{R}_{+}^{\mathcal{A}}
$$

Seja $\zeta^{n}=\left(\zeta_{\alpha}^{n}\right)_{\alpha \in \mathcal{A}}$ o vetor partição de $f_{n}=R^{n}(f)$, ou seja $\zeta_{\alpha}^{n}=\left|I_{\alpha}^{n}\right|$. Agora, para todo $n \geq 0$ defina o operador linear $T_{n}: \mathbb{R}^{\mathcal{A}} \rightarrow \mathbb{R}^{\mathcal{A}}$ cuja a matriz é dada por

$$
T_{n}=\left(T_{n}\right)_{i j}= \begin{cases}1, & \text { se } i=j \\ \exp \left(L_{n, \alpha^{n}(1-\varepsilon)}\right), & \text { se } i=\alpha^{n}(\varepsilon), j=\alpha^{n}(1-\varepsilon) \\ 0, & \text { caso contrário, }\end{cases}
$$

onde $L_{n, \alpha^{n}(1-\varepsilon)}$ é definido por (6.27).

Lema 6.4.2. Seja $f \in \mathcal{B}_{k}^{2+\nu} \operatorname{com} \int D^{2} f(s) / D f(s) d s=0$. Then for all $n \geq 0$

$$
T_{n} \zeta^{n+1}=\zeta^{n}+\mathrm{O}\left(\lambda_{5}^{\sqrt{n}}\right) .
$$


Demonstração. Suponhamos que $R^{n} f$ tem tipo 0, o caso em que $R^{n} f$ tem tipo 1 é similar. Então

$$
\zeta_{\alpha}^{n+1}= \begin{cases}\zeta_{\alpha}^{n}, & \text { se } \alpha \neq \alpha^{n}(0) \\ \zeta_{\alpha^{n}(0)}^{n}-\left|R^{n} f\left(I_{\alpha^{n}(1)}^{n}\right)\right|, & \text { se } \alpha=\alpha^{n}(0)\end{cases}
$$

Pelo Teorema do valor médio sabemos que $\left|R^{n} f\left(I_{\alpha^{n}(1)}^{n}\right)\right|=D R^{n} f(x) \cdot \zeta_{\alpha^{n}(1)}^{n}$, para algum $x \in I_{\alpha^{n}(1)}^{n}$. Temos também, pela definição de $L_{n, \alpha^{n}(1)}$ que

$$
\ln D R^{n} f(x)=L_{n, \alpha^{n}(1)}+\mathrm{O}\left(\lambda_{5}^{\sqrt{n}}\right) \Leftrightarrow D R^{n} f(x)=\exp \left(L_{n, \alpha^{n}(1)}\right)+\mathrm{O}\left(\lambda_{5}^{\sqrt{n}}\right)
$$

e portanto o resultado segue.

Note que $\left(T_{n}\right)_{i j} \geq 0$ para todo $n \geq 0$. Então nós podemos definir a projetivização of $T_{n}$, isto é, a aplicação $T_{n}^{\text {nor }}: \Delta_{\mathcal{A}} \rightarrow \Delta_{\mathcal{A}}$ dada por

$$
T_{n}^{\text {nor }} \zeta^{n+1}=\frac{T_{n} \zeta^{n+1}}{\left\|T_{n} \zeta^{n+1}\right\|_{1}}
$$

Agora, seja $f_{A}$ o modelo afim de $f$ e $\tilde{\zeta}^{n}=\left(\tilde{\zeta}_{\alpha}^{n}\right)_{\alpha \in \mathcal{A}}$ o vetor partição de $R^{n} f_{A}$. Analogamente definimos $\tilde{T}_{n}$ e $\tilde{T}_{n}^{\text {nor }}$ por

$$
\tilde{T}_{n}=\left(\tilde{T}_{n}\right)_{i j}= \begin{cases}1, & \text { se } i=j \\ \exp \left(\omega_{\alpha^{n}(1-\varepsilon)}^{n}\right), & \text { se } i=\alpha^{n}(\varepsilon), j=\alpha^{n}(1-\varepsilon) \\ 0, & \text { caso contrário }\end{cases}
$$

e

$$
\tilde{T}_{n}^{\text {nor }} \zeta^{n+1}=\frac{\tilde{T}_{n} \zeta^{n+1}}{\left\|\tilde{T}_{n} \zeta^{n+1}\right\|_{1}}
$$

Lema 6.4.3. Sejam $f \in \mathcal{B}_{k}^{2+\nu} \operatorname{com} \int D^{2} f(s) / D f(s) d s=0, f_{A}$ seu modelo afim e $\zeta^{n}, \tilde{\zeta}^{n} \in \Delta_{A}$ os vetores partições de $R^{n} f$ e $R^{n} f_{A}$ respectivamente. Então para todo $n \geq 0$

$$
\zeta^{n}=\tilde{T}_{n}^{\text {nor }} \zeta^{n+1}+\mathrm{O}\left(\lambda_{5}^{\sqrt{n}}\right)
$$

Demonstração.

$$
\left|\zeta^{n}-\tilde{T}_{n}^{\text {nor }}\left(\zeta^{n+1}\right)\right| \leq\left|\zeta^{n}-T_{n}^{\text {nor }} \zeta^{n+1}\right|+\left|T_{n}^{\text {nor }} \zeta^{n+1}-\tilde{T}_{n}^{\text {nor }} \zeta^{n+1}\right|
$$


O primeiro termo acima é da ordem $\mathrm{O}\left(\lambda_{5}^{\sqrt{n}}\right)$, pelos Lemas 6.4.2, 4.0.6 e 4.0.7. Suponhamos sem perda que $R^{n} f$ é do tipo 0. Então

$$
\begin{aligned}
T_{n}^{\text {nor }} \zeta^{n+1}-\tilde{T}_{n}^{\text {nor }} \zeta^{n+1}= & \frac{T_{n} \zeta^{n+1}}{1-\left|R^{n} f\left(I_{\alpha^{n}(1)}^{n}\right)\right|}-\frac{\tilde{T}_{n} \zeta^{n+1}}{1-\exp \omega_{\alpha^{n}(1)}^{n}\left|I_{\alpha^{n}(1)}^{n}\right|} \\
= & \frac{T_{n} \zeta^{n+1}}{1-\left|R^{n} f\left(I_{\alpha^{n}(1)}^{n}\right)\right|}-\frac{\zeta^{n}}{1-\left|R^{n} f\left(I_{\alpha^{n}(1)}^{n}\right)\right|} \\
& +\frac{\zeta^{n}}{1-\left|R^{n} f\left(I_{\alpha^{n}(1)}^{n}\right)\right|}-\frac{\tilde{T}_{n} \zeta^{n+1}}{1-\exp \omega_{\alpha^{n}(1)}^{n}\left|I_{\alpha^{n}(1)}^{n}\right|}
\end{aligned}
$$

A primeira parcela da soma acima é estimada por $\mathrm{O}\left(\lambda_{5}^{\sqrt{n}}\right)$, pelo Lema 6.4.2. Pelo Lema 6.3.4 temos que $\tilde{T}_{n} \zeta^{n+1}=T_{n} \zeta^{n+1}+\mathrm{O}\left(\lambda_{5}^{\sqrt{n}}\right.$. Disto e dos Lemas 4.0.6, 4.0.7 segue que a segunda parcela também é estimada por $\mathrm{O}\left(\lambda_{5}^{\sqrt{n}}\right)$.

Dado $n, m \in \mathbb{N}$ com $n<m$ defina

$$
\mathcal{T}_{n}^{m}:=\tilde{T}_{n}^{\text {nor }} \cdots \tilde{T}_{n}^{\text {nor }}
$$

Pela definição de $\tilde{T}_{n}^{\text {nor }}$ sabemos que

$$
\tilde{T}_{n}^{\text {nor }}>0 \Leftrightarrow \Theta_{\pi^{n}, \varepsilon^{n}}>0 .
$$

Então por [MMY05a, Seção 1.2.4] temos que para todo $n \geq k(2 d-3)$

$$
\mathcal{T}_{n-k(2 d-3)}^{n-1}>0
$$

e portanto $\mathcal{T}_{n-k(2 d-3)}^{n-1}$ é uma contração na métrica projetiva. A constante de contração será denotada por $\kappa$.

Seja $K \subset \Delta_{\mathcal{A}}$ o conjunto de todos $v \in \Delta_{\mathcal{A}}$ tal que existe $c>0$ com $v_{\alpha} \geq c>0$ para todo $\alpha \in \mathcal{A}$. Então pelos Lemas (4.0.6) e (4.0.7), $\zeta^{n}, \tilde{\zeta^{n}} \in K$ para todo $n \geq 0$. $K$ é relativamente compacto em $\Delta_{\mathcal{A}}$ e por definição de $\mathrm{d}_{p}$

$$
\operatorname{diam}(K)=\sup _{x, y \in K} \mathrm{~d}_{p}(x, y)<\infty
$$

Lema 6.4.4. A métrica $\mathrm{d}_{p}$ é equivalente a norma da soma em $K$. 
Demonstração. Seja $\theta, \gamma \in K$. Então

$$
\begin{aligned}
\mathrm{d}_{p}(\theta, \gamma) & =\log \frac{\theta_{\alpha} \gamma_{\beta}}{\gamma_{\alpha} \theta_{\beta}} \text { para algum } \alpha, \beta \in \mathcal{A} \\
& \leq\left|\log \theta_{\alpha}-\log \gamma_{\alpha}\right|+\left|\log \theta_{\beta}-\log \gamma_{\beta}\right| \\
& \leq \frac{1}{x_{\alpha}}\left|\theta_{\alpha}-\gamma_{\alpha}\right|+\frac{1}{x_{\beta}}\left|\theta_{\alpha}-\gamma_{\alpha}\right|, \text { pelo Teorema do Valor Médio } \\
& \leq \frac{1}{c}\left(\left|\theta_{\alpha}-\gamma_{\alpha}\right|+\left|\theta_{\beta}-\gamma_{\beta}\right|\right) \\
& \leq \frac{1}{c}|\theta-\gamma|_{1} .
\end{aligned}
$$

Suponhamos sem perda de generalidade que $\log \theta_{\alpha}-\log \gamma_{\alpha}>0$ e $\log \theta_{\beta}-\log \gamma_{\beta}>0$. Então

$$
\begin{aligned}
|\theta-\gamma|_{1} & \leq d\left(\left|\theta_{\alpha}-\gamma_{\alpha}\right|+\left|\theta_{\beta}-\gamma_{\beta}\right|\right) \\
& =d \cdot x_{\alpha} \cdot\left(\log \theta_{\alpha}-\log \gamma_{\alpha}\right)+d \cdot x_{\beta} \cdot\left(\log \theta_{\beta}-\log \gamma_{\beta}\right) \\
& =C \cdot \log \frac{\theta_{\alpha} \gamma_{\beta}}{\gamma_{\alpha} \theta_{\beta}} \\
& \leq \mathrm{d}_{p}(\theta, \gamma) .
\end{aligned}
$$

Agora comparamos na métrica projetiva os vetores partição $\zeta^{n}, \tilde{\zeta}^{n}$ de $R^{n} f$ e $R^{n} f_{A}$ respectivamente. Para isto, precisaremos de algumas estimativas que serão dadas a seguir.

$$
\begin{aligned}
\mathrm{d}_{p}\left(\zeta^{n-k(2 d-3)}, \mathcal{T}_{n-k(2 d-3)}^{n-1} \zeta^{n}\right) \leq & \mathrm{d}_{p}\left(\zeta^{n-k(2 d-3)}, \tilde{T}_{n-k(2 d-3)}^{\text {nor }} \zeta^{n-k(2 d-3)+1}\right) \\
& +\mathrm{d}_{p}\left(\tilde{T}_{n-k(2 d-3)}^{\text {nor }} \zeta^{n-k(2 d-3)+1}, \mathcal{T}_{n-k(2 d-3)}^{n-1} \zeta^{n}\right) \\
\leq & \mathrm{O}\left(\lambda_{5}^{\sqrt{n-k(2 d-3)}}\right)+\mathrm{d}_{p}\left(\zeta^{n-k(2 d-3)+1}, \mathcal{T}_{n-k(2 d-3)+1}^{n-1} \zeta^{n}\right) .
\end{aligned}
$$

Aplicando esse processo $k(2 d-3)$-vezes, obtemos

$$
\mathrm{d}_{p}\left(\zeta^{n-k(2 d-3)}, \mathcal{T}_{n-k(2 d-3)}^{n-1} \zeta^{n}\right) \leq \sum_{i=0}^{k(2 d-3)-1} \mathrm{O}\left(\lambda_{5}^{\sqrt{n-k(2 d-3)+i}}\right)
$$

mas

$$
\begin{aligned}
\sum_{i=0}^{k(2 d-3)-1} \mathrm{O}\left(\lambda_{5}^{\sqrt{n-k(2 d-3)+i}}\right) & =\mathrm{O}\left(\lambda_{5}^{\sqrt{n-k(2 d-3)}}\right) \sum_{i=0}^{k(2 d-3)-1} \mathrm{O}\left(\lambda_{5}^{\sqrt{n-k(2 d-3)+i}-\sqrt{n-k(2 d-3)}}\right) \\
& \leq k(2 d-3) \cdot \mathrm{O}\left(\lambda_{5}^{\sqrt{n-k(2 d-3)}}\right) \\
& =\mathrm{O}\left(\lambda_{5}^{\sqrt{n-k(2 d-3)}}\right)
\end{aligned}
$$


De (6.39) e (6.40) obtemos

$$
\begin{aligned}
& \mathrm{d}_{p}\left(\zeta^{n-k(2 d-3)}, \mathcal{T}_{n-k(2 d-3)}^{n-1} \zeta^{n}\right)=\mathrm{O}\left(\lambda_{5}^{\sqrt{n-k(2 d-3)}}\right) . \\
& d_{p}\left(\zeta^{n-2 k(2 d-3)}, \mathcal{T}_{n-2 k(2 d-3)}^{n-1} \zeta^{n}\right) \leq d_{p}\left(\zeta^{n-2 k(2 d-3)}, \mathcal{T}_{n-2 k(2 d-3)}^{n-k(2 d)-1} \zeta^{n-k(2 d-3)}\right) \\
&+\mathrm{d}_{p}\left(\mathcal{T}_{n-2 k(2 d-3)}^{n-k(2 d-3)} \zeta^{n-k(2 d-3)}, \mathcal{T}_{n-2 k(2 d-3)}^{n-1} \zeta^{n}\right) \\
& \leq \mathrm{O}\left(\lambda_{5}^{\sqrt{n-2 k(2 d-3)}}\right)+\kappa \cdot \mathrm{d}_{p}\left(\zeta^{n-k(2 d-3)}, \mathcal{T}_{n-k(2 d-3)}^{n-1} \zeta^{n}\right) \\
&= \mathrm{O}\left(\lambda_{5}^{\sqrt{n-2 k(2 d-3)}}\right)+\kappa \cdot \mathrm{O}\left(\lambda_{5}^{\sqrt{n-k(2 d-3)}}\right), \text { por }(6.41) .
\end{aligned}
$$

Aplicando esse argumento $j$-vezes, obtemos

$$
\begin{aligned}
d_{p}\left(\zeta^{n-j k(2 d-3)}, \mathcal{T}_{n-j k(2 d-3)}^{n-1} \zeta^{n}\right) & =\sum_{i=0}^{j-1} \kappa^{j-1-i} \mathrm{O}\left(\lambda_{5}^{\sqrt{n-(i+1) k(2 d-3)}}\right) \\
& \leq \mathrm{O}\left(\lambda_{5}^{\sqrt{n-j k(2 d-3)}}\right) \cdot \sum_{i=0}^{j-1} \kappa^{j-1-i} \\
& \leq \frac{1}{1-\kappa} \cdot \mathrm{O}\left(\lambda_{5}^{\sqrt{n-j k(2 d-3)}}\right) \\
& =\mathrm{O}\left(\lambda_{5}^{\sqrt{n-j k(2 d-3)}}\right)
\end{aligned}
$$

Agora estamos prontos para comparar na métrica projetiva os vetores $\zeta^{n}, \tilde{\zeta}^{n}$.

$$
\begin{aligned}
\mathrm{d}_{p}\left(\zeta^{n-j k(2 d-3)}, \tilde{\zeta}^{n-j k(2 d-3)}\right) \leq & \mathrm{d}_{p}\left(\zeta^{n-j k(2 d-3)}, \mathcal{T}_{n-j k(2 d-3)}^{n-1} \zeta^{n}\right) \\
& +\mathrm{d}_{p}\left(\mathcal{T}_{n-j k(2 d-3)}^{n-1} \zeta^{n}, \mathcal{T}_{n-j k(2 d-3)}^{n-1} \tilde{\zeta}^{n}\right) \\
\leq & \mathrm{O}\left(\lambda_{5}^{\sqrt{n-j k(2 d-3)}}\right)+\kappa^{j k(2 d-3)} \cdot \mathrm{d}_{p}\left(\zeta^{n}, \tilde{\zeta}^{n}\right) \\
\leq & \mathrm{O}\left(\lambda_{5}^{\sqrt{n-j k(2 d-3)}}\right)+\kappa^{j k(2 d-3)} \cdot \operatorname{diam}(K)
\end{aligned}
$$

Tomando $j=\left[\frac{n}{2 k(2 d-3)}\right]$ em (6.43) temos

$$
\mathrm{d}_{p}\left(\zeta^{[n / 2]}, \tilde{\zeta}^{[n / 2]}\right) \leq \mathrm{O}\left(\lambda_{5}^{\sqrt{[n / 2]}}\right)+\kappa^{[n / 2]} \cdot \operatorname{diam}(K)
$$

e portanto

$$
\mathrm{d}_{p}\left(\zeta^{n}, \tilde{\zeta}^{n}\right)=\mathrm{O}\left(\lambda_{5}^{\sqrt{n}}\right)
$$

O Lema 6.4.4 junto com (6.44) nos dá 


$$
\left\|\zeta^{n}-\tilde{\zeta}^{n}\right\|_{1}=\mathrm{O}\left(\lambda_{5}^{\sqrt{n}}\right)
$$

Agora estimaremos a diferença entre os comprimentos de $R^{n} f\left(I_{\alpha}^{n}\right)$ e $R^{n} f_{A}\left(\tilde{I}_{\alpha}\right)$ para todo $\alpha \in \mathcal{A}$. Primeiro note que pelo Lema 6.3.4

$$
\frac{\omega_{\alpha}^{n}}{L_{n, \alpha}}=\exp \left(\mathrm{O}\left(\lambda_{5}^{\sqrt{n}}\right)\right) \Leftrightarrow 1-\frac{\omega_{\alpha}^{n}}{L_{n, \alpha}}=\mathrm{O}\left(\lambda_{5}^{\sqrt{n}}\right)
$$

Para todo $\alpha \in \mathcal{A}$ sejam $x_{\alpha}^{n} \in I_{\alpha}^{n}$ e $\tilde{x}_{\alpha}^{n} \in \tilde{I}_{\alpha}^{n}$ tal que

$$
\left|R^{n} f\left(I_{\alpha}^{n}\right)\right|=D R^{n} f\left(x_{\alpha}^{n}\right) \cdot \zeta_{\alpha}^{n} \text { e }\left|R^{n} f_{A}\left(\tilde{I}_{\alpha}^{n}\right)\right|=D R^{n} f_{A}\left(\tilde{x}_{\alpha}^{n}\right) \cdot \tilde{\zeta}_{\alpha}^{n} .
$$

Então

$$
\begin{aligned}
\left|R^{n} f\left(I_{\alpha}^{n}\right)\right|-\left|R^{n} f_{A}\left(\tilde{I}_{\alpha}\right)\right| & =D R^{n} f\left(x_{\alpha}^{n}\right) \cdot \zeta_{\alpha}^{n}-D R^{n} f_{A}\left(\tilde{x}_{\alpha}^{n}\right) \cdot \tilde{\zeta}_{\alpha}^{n} \\
& =\left[\frac{D R^{n} f\left(x_{\alpha}^{n}\right)}{D R^{n} f_{A}\left(x_{\alpha}^{n}\right)} \cdot \zeta_{\alpha}^{n}-\tilde{\zeta}_{\alpha}^{n}\right] \cdot D R^{n} f_{A}\left(x_{\alpha}^{n}\right) \\
& =\left[\frac{L_{n, \alpha}}{\omega_{\alpha}^{n}} \cdot \zeta_{\alpha}^{n}-\tilde{\zeta}_{\alpha}^{n}+\mathrm{O}\left(\lambda_{5}^{\sqrt{n}}\right)\right] \cdot \omega_{\alpha}^{n} \\
& =\left[\frac{L_{n, \alpha}}{\omega_{\alpha}^{n}} \cdot \zeta_{\alpha}^{n} \pm \frac{L_{n, \alpha}}{\omega_{\alpha}^{n}} \cdot \tilde{\zeta}_{\alpha}^{n}-\tilde{\zeta}_{\alpha}^{n}+\mathrm{O}\left(\lambda_{5}^{\sqrt{n}}\right)\right] \cdot \omega_{\alpha}^{n} \\
& =\left[\frac{L_{n, \alpha}}{\omega_{\alpha}^{n}} \cdot\left(\zeta_{\alpha}^{n}-\tilde{\zeta}_{\alpha}^{n}\right)+\left(\frac{L_{n, \alpha}}{\omega_{\alpha}^{n}}-1\right)+\mathrm{O}\left(\lambda_{5}^{\sqrt{n}}\right)\right] \cdot \omega_{\alpha}^{n} \\
& =\mathrm{O}\left(\lambda_{5}^{\sqrt{n}}\right),
\end{aligned}
$$

por $(6.45)$ e (6.46).

De (6.37), (6.45) e (6.47) concluímos que

$$
\mathrm{d}_{C^{2}}\left(R^{n} f, R^{n} f_{A}\right)=\mathrm{O}\left(\lambda_{5}^{\sqrt{n}}\right)
$$

E portanto o Teorema E está demosntrado.

\subsection{Prova do Teorema A}

Nesta seção nós provaremos o Teorema A., que reenunciaremos a seguir.

Teorema A. Sejam $f, g: \mathbb{S}^{1} \rightarrow \mathbb{S}^{1}$ homeomorfismos do círculo, $C^{2+\nu}$ por pedaços tais que 
- $f$ e g nãa tem conexão;

- $f$ e g possuem a mesma combinatória $k$-limitada;

- $f$ e g são break-equivalentes;

- $\int_{0}^{1} \frac{D^{2} f(s)}{D f(s)} d s=\int_{0}^{1} \frac{D^{2} g(s)}{D g(s)} d s=0$.

Então existe uma conjugação de classe $C^{1}$ entre $f$ e $g$.

Como $f$ e $g$ possuem a mesma combinatória e são homeomorfismos de classe $\mathrm{P}$, temos que $f$ e $g$ são conjugadas. Seja $h: \mathbb{S}^{1} \rightarrow \mathbb{S}^{1}$ a conjugação que leva break-point de $f$ em break-point de $g$, isto é

$$
g \circ h=h \circ f .
$$

A equação cohomologica associada a (6.49) é dada por

$$
\ln D g \circ h-\ln D f=\psi \circ f-\psi,
$$

onde $\psi:[0,1] \rightarrow \mathbb{R}$ é chamada solução de (6.50), caso exista.

Como é sabido da teoria de difeomorfismos do círculo, mostrar que a conjugação $h$ é $C^{1}$ é equivalente a mostrar que a equação (6.50) admite uma solução contínua. Nossos próximos resultados são nesta direção.

Para todo $x \in[0,1)$ defina $i_{n}(x):=\min \left\{i \geq 0 ; f^{i}(x) \in I^{n}\right\}$, e defina a função $\psi_{n}:[0,1) \rightarrow \mathbb{R}$ por

$$
\psi_{n}(x):=\sum_{i=0}^{i_{n}(x)-1} \ln D f\left(f^{i}(x)\right)-\ln D g\left(h \circ f^{i}(x)\right) .
$$

Lema 6.5.1. Para cada $x \in[0,1)$ a função $\psi_{n}$ converge uniformemente em cada elemento da partição $\mathcal{P}^{n}$.

Demonstração. Nós mostraremos que $\psi_{n}$ é de Cauchy. Suponha que $i_{n}(x) \neq i_{n+1}(x)$, ou seja $i_{n}(x)<i_{n+1}(x)$. Pela definição de $i_{n}(x)$ temos que $i_{n+1}(x)=i_{n}(x)+q_{\left(\pi_{0}^{n}\right)^{-1}(d)}^{n}$. Portanto,

$$
\begin{aligned}
& \psi_{n+1}(x)-\psi_{n}(x)=\sum_{i=i_{n}(x)}^{i_{n+1}(x)-1} \ln D f\left(f^{i}(x)\right)-\ln D g\left(h \circ f^{i}(x)\right) \\
& =\ln D f^{i_{n+1}(x)-i_{n}(x)}\left(f^{i_{n}(x)}(x)\right)-\ln D g^{i_{n+1}(x)-i_{n}(x)}\left(h \circ f^{i_{n}(x)}(x)\right) \\
& =\ln D f^{q_{\left(\pi_{0}^{n}\right)-1}^{n}(d)}\left(f^{i_{n}(x)}(x)\right)-\ln D g^{q_{\left(\pi_{0}^{n}\right)-1}^{n}(d)}\left(h \circ f^{i_{n}(x)}(x)\right) \\
& =\ln D R^{n} f\left(f^{i_{n}(x)}(x)\right)-\ln D R^{n} g\left(h \circ f^{i_{n}(x)}(x)\right) \\
& =\mathrm{O}\left(\lambda_{5}^{\sqrt{n}}\right), \text { pela Proposição 6.3.4. }
\end{aligned}
$$


Note que a função $i_{n}:[0,1) \rightarrow \mathbb{N}$ é constante e portanto contínua em cada elemento da partição $\mathcal{P}^{n}$. Portanto $\psi_{n}$ converge uniformemente em cada elemento da partição.

Seja $\psi(x)=\lim _{n \rightarrow \infty} \psi_{n}(x)$.

Lema 6.5.2. $\psi:[0,1) \rightarrow \mathbb{R}$ é solução da equação (6.50) e é contínua.

Demonstração. Para verificar que $\psi$ é solução da equação (6.50), basta notar que $i_{n}(x)=i_{n}(f(x))+1$. Seja $I_{\beta}$ o subintervalo, tal que $f\left(\partial I_{\beta}\right)=0$. Defina

$$
f_{+}(x)=\lim _{y \rightarrow x_{+}} f(y) \text { and } f_{-}(x)=\lim _{y \rightarrow x_{-}} f(y) .
$$

Seja $\mathcal{O}_{+}(x)=\left\{x, f_{+}(x), \ldots, f_{+}^{n}(x), \ldots\right\}$ e $\mathcal{O}_{-}(x)=\left\{x, f_{-}(x), \ldots, f_{-}^{n}(x), \ldots\right\}$. Dado $x \in[0,1)$ tome $k_{0}$ minimal, tal que $f^{k_{0}}(x)=\partial I_{\beta}$. Então $f_{+}^{i}(x)=f_{-}^{i}(x)$ para todo $i \geq 0$, exceto $i=k_{0}+1$. Seja $n_{0} \in \mathbb{N}$ tal que $f(0) \notin \overline{I^{n_{0}}}$. Então para todo $n>n_{0}$ e para todo $x \in[0,1), i_{n}\left(x_{+}\right)=i_{n}\left(x_{-}\right)$temos

$$
\begin{aligned}
\psi\left(x_{+}\right)-\psi\left(x_{-}\right)= & \lim _{n \rightarrow \infty} \psi_{n}\left(x_{+}\right)-\lim _{n \rightarrow \infty} \psi_{n}\left(x_{-}\right) \\
= & \ln D f\left(f_{+}^{k_{0}+1}(x)\right)-\ln D g\left(h \circ f_{+}^{k_{0}+1}(x)\right) \\
& -\ln D f\left(h \circ f_{-}^{k_{0}+1}(x)\right)+\ln D g\left(f_{-}^{k_{0}+1}(x)\right) \\
= & \ln \frac{D f\left(f_{+}^{k_{0}+1}(x)\right)}{D f\left(f_{-}^{k_{0}+1}(x)\right)}-\ln \frac{D g\left(h \circ f_{+}^{k_{0}+1}(x)\right)}{D g\left(h \circ f_{-}^{k_{0}+1}(x)\right)} \\
= & 0,
\end{aligned}
$$

pois $f$ e $g$ são break-equivalentes..

Nosso próximo passo é mostrar que a conjugação $h$ é Lipschitz. Mas para isso precisamos do seguinte lema.

Lema 6.5.3. Existe constante $C_{31}>0$, tal que para todo $\beta \in \mathcal{A}$

$$
\lim _{n \rightarrow \infty} \frac{\left|R^{n} g\left(h\left(I_{\beta}^{n}\right)\right)\right|}{\left|R^{n} f\left(I_{\beta}^{n}\right)\right|}=C_{31} .
$$

Demonstração.

$$
\begin{aligned}
\ln \frac{\left|R^{n} g\left(h\left(I_{\beta}^{n}\right)\right)\right|}{\left|R^{n} f\left(I_{\beta}^{n}\right)\right|} & =\ln \frac{D R^{n} g(h(y))}{D R^{n} f(y)}+\ln \frac{\left|h\left(I_{\beta}^{n}\right)\right|}{\left|I_{\beta}^{n}\right|} \\
& =\mathrm{O}\left(\lambda_{5}^{\sqrt{n}}\right)+\ln \frac{\left|h\left(I_{\beta}^{n}\right)\right|}{\left|I_{\beta}^{n}\right|}
\end{aligned}
$$


Agora estimaremos o segundo termo. Seja $\zeta, \tilde{\zeta}$ os vetores partições de $R^{n} f$ e $R^{n} g$, respectivamente. Então

$$
\frac{\left|I_{\beta}^{n}\right|}{\left|I^{n}\right|}=\frac{\left|h\left(I_{\beta}^{n}\right)\right|}{\left|h\left(I^{n}\right)\right|}+\mathrm{O}\left(\lambda_{5}^{\sqrt{n}}\right) .
$$

De fato,

$$
\begin{aligned}
\frac{\left|I_{\beta}^{n}\right|}{\left|I^{n}\right|}-\frac{\left|h\left(I_{\beta}^{n}\right)\right|}{\left|h\left(I^{n}\right)\right|} & =\frac{\left|I_{\beta}^{n}\right|}{\left|I^{n}\right|} \pm \frac{\left|h\left(I_{\beta}^{n}\right)\right|}{\left|I^{n}\right|}-\frac{\left|h\left(I_{\beta}^{n}\right)\right|}{\left|h\left(I^{n}\right)\right|} \\
& =\frac{\zeta_{\beta}^{n}-\tilde{\zeta}_{\beta}^{n}}{\left|I^{n}\right|}+\frac{\tilde{\zeta}_{\beta}^{n} \cdot\left(\left|h\left(I^{n}\right)\right|-\left|I^{n}\right|\right)}{\left|h\left(I^{n}\right)\right| \cdot\left|I^{n}\right|} \\
& =\frac{\zeta^{n} n_{\beta}-\tilde{\zeta}_{\beta}^{n}}{\sum_{\alpha \in \mathcal{A}} \zeta_{\alpha}^{n}}+\frac{\tilde{\zeta}_{\beta}^{n} \cdot\left(\left|h\left(I^{n}\right)\right|-\left|I^{n}\right|\right)}{\sum_{\alpha \in \mathcal{A}} \zeta_{\alpha}^{n} \cdot \sum_{\alpha \in \mathcal{A}} \tilde{\zeta}_{\alpha}^{n}} .
\end{aligned}
$$

Usando o fato de que $\zeta_{\alpha}^{n} \geq c>0$ para todo $n \geq 0$ e para todo $\alpha \in \mathcal{A}$ e a estimativa (6.45), concluímos que a primeira parcela acima é estimada por $\mathrm{O}\left(\lambda_{5}^{\sqrt{n}}\right)$. Usando o argumento anterior e o Lema 4.0.6 concluímos que a segunda parcela também é estimada por $\mathrm{O}\left(\lambda_{5}^{\sqrt{n}}\right)$.

Da equação (6.52) e pelo Lema 6.3.4 temos que

$$
\frac{\left|R^{n} f\left(I_{\beta}^{n}\right)\right|}{\left|I^{n}\right|}=\frac{\left|R^{n} f_{A}\left(h\left(I_{\beta}^{n}\right)\right)\right|}{\left|h\left(I^{n}\right)\right|}+\mathrm{O}\left(\lambda_{5}^{\sqrt{n}}\right) .
$$

Agora, sem perda de generalidade, suponha que $R^{n} f$ é de tipo 0. Então

$$
\begin{aligned}
\frac{\left|I^{n+1}\right|}{\left|I^{n}\right|} & =\frac{\left|I^{n}\right|-\left|R^{n} f\left(I_{\alpha^{n}(1)}^{n}\right)\right|}{\left|I^{n}\right|} \\
& =1-\frac{\left|R^{n} f\left(I_{\alpha^{n}(1)}^{n}\right)\right|}{\left|I^{n}\right|} \\
& =1-\frac{\mid R^{n} f_{A}\left(h\left(I_{\alpha^{n}(1)}^{n}\right) \mid\right.}{\left|h\left(I^{n}\right)\right|}+\mathrm{O}\left(\lambda_{5}^{\sqrt{n}}\right) \text { por }(6.53) \\
& =\frac{\left|h\left(I^{n+1}\right)\right|}{\left|h\left(I^{n}\right)\right|}+\mathrm{O}\left(\lambda_{5}^{\sqrt{n}}\right) .
\end{aligned}
$$

De (6.54) obtemos

$$
\frac{\left|h\left(I^{n+1}\right)\right|}{\left|h\left(I^{n}\right)\right|}=\left(1+\mathrm{O}\left(\lambda_{5}^{\sqrt{n}}\right)\right) \cdot \frac{\left|I^{n+1}\right|}{\left|I^{n}\right|} .
$$

Assim

$$
\begin{aligned}
\ln \frac{\left|h\left(I^{n}\right)\right|}{\left|I^{n}\right|} & =\ln \frac{\left|h\left(I^{0}\right)\right|}{\left|I^{0}\right|}+\sum_{i=1}^{n} \ln \left[\frac{\left|h\left(I^{i}\right)\right|}{\left|h\left(I^{i-1}\right)\right|} \cdot \frac{\left|I^{i-1}\right|}{\left|I^{i}\right|}\right] \\
& =\ln \frac{\left|h\left(I^{0}\right)\right|}{\left|I^{0}\right|}+\sum_{i=1}^{n} \ln \left[1+\mathrm{O}\left(\lambda_{5}^{\sqrt{i-1}}\right)\right] .
\end{aligned}
$$


Como a série acima é somável, temos que existe constante $C_{31}$ tal que

$$
\lim _{n \rightarrow \infty} \ln \frac{\left|h\left(I^{n}\right)\right|}{\left|I^{n}\right|}=C_{31}
$$

Sabendo que

$$
\begin{aligned}
\ln \frac{\left|h\left(I_{\beta}^{n}\right)\right|}{\left|I_{\beta}^{n}\right|} & =\ln \frac{\left|h\left(I_{\beta}^{n}\right)\right|}{\left|h\left(I^{n}\right)\right|}+\ln \frac{\left|h\left(I^{n}\right)\right|}{\left|I^{n}\right|}+\ln \frac{\left|I^{n}\right|}{\left|I_{\beta}^{n}\right|} \\
& =\ln \left(1+\mathrm{O}\left(\lambda_{5}^{\sqrt{n}}\right)\right)+\ln \frac{\left|h\left(I^{n}\right)\right|}{\left|I^{n}\right|}, \quad \text { by (6.52), }
\end{aligned}
$$

temos

$$
\lim _{n \rightarrow \infty} \ln \frac{\left|h\left(I_{\beta}^{n}\right)\right|}{\left|I_{\beta}^{n}\right|}=C_{31}, \text { by }(6.56)
$$

O resultado é obtido, de (6.57), tomando o limite quando $n \rightarrow \infty$ em (6.51) .

Lema 6.5.4. A conjugação $h:[0,1) \rightarrow[0,1)$ é Lipschitz.

Demonstração. Primeiro nós mostramos que $h$ é Lipschitz em cada elemento da partição $\mathcal{P}^{n}$. Seja $J_{n} \in \mathcal{P}^{n}$. Então $R^{n} f\left(J_{n}\right)=f^{i_{n}}\left(J_{n}\right)$ e $R^{n} g\left(h\left(J_{n}\right)\right)=g^{i_{n}}\left(h\left(J_{n}\right)\right)$. Sabemos que

$$
\begin{aligned}
\frac{\left|h\left(J_{n}\right)\right|}{\left|J_{n}\right|} & =\frac{D f^{i_{n}}(y)}{D f_{A}^{i_{n}}(h(y))} \cdot \frac{\left|R^{n} f_{A}\left(h\left(J_{n}\right)\right)\right|}{\left|R^{n} f\left(J_{n}\right)\right|} \cdot \exp \left(\mathrm{O}\left(\lambda_{5}^{\sqrt{n}}\right)\right) \\
& =e^{\psi_{n}(y)} \cdot \frac{\left|R^{n} f_{A}\left(h\left(J_{n}\right)\right)\right|}{\left|R^{n} f\left(J_{n}\right)\right|} \cdot \exp \left(\mathrm{O}\left(\lambda_{5}^{\sqrt{n}}\right)\right),
\end{aligned}
$$

onde $y \in J_{n}$. Tomando $C_{32}=\sup _{y \in[0,1)}\left\{e^{\psi\left(y_{n}\right)}\right\}$ e fazendo $n \rightarrow \infty$ temos

$$
\lim _{n \rightarrow \infty} \frac{\left|h\left(J_{n}\right)\right|}{\left|J_{n}\right|}=C_{31} \cdot C_{32}, \text { pelo Lema 6.5.3. }
$$

Portanto existe constante $C_{33}$ tal que

$$
\left|h\left(J_{n}\right)\right| \leq C_{33} \cdot\left|J_{n}\right|
$$

Seja $x, y \in[0,1)$ tal que $x<y$ e $A=[x, y)$. Defina

$$
\mathcal{F}_{1}=\left\{J_{1} \in \mathcal{P}^{1} \backslash J_{1}^{s} \subset A\right\}=\left\{J_{1}^{1}, \ldots, J_{1}^{s_{1}}\right\}
$$

e

$$
\mathcal{F}_{n}=\left\{J_{n} \in \mathcal{P}^{n} \backslash J_{n} \cap \mathcal{F}_{n-1}=\varnothing \text { e } J_{n} \subset A\right\}=\left\{J_{n}^{1}, \ldots, J_{n}^{s_{n}}\right\},
$$


onde $J_{n} \cap \mathcal{F}_{n-1}=$ se e somente se $J_{n} \cap F=$ para todo $F \in \mathcal{F}_{n-1}$. Note que se $n \neq m$ então $J_{n}^{s} \cap J_{m}^{r}=\varnothing$ para todo $1 \leq s \leq s_{n}$ e para todo $1 \leq r \leq s_{m}$.

É claro que

$$
\bigcup_{i=1}^{n} \bigcup_{j=1}^{s_{i}} J_{i}^{j} \nearrow A \text { e } \bigcup_{i=1}^{n} \bigcup_{j=1}^{s_{i}} h\left(J_{i}^{j}\right) \nearrow h(A) .
$$

Portanto

$$
\begin{aligned}
|h(A)| & =\sum_{i=1}^{n} \sum_{j=1}^{s_{i}}\left|h\left(J_{i}^{j}\right)\right| \\
& \leq C_{33} \cdot \sum_{i=1}^{n} \sum_{j=1}^{s_{i}}\left|J_{i}^{j}\right| \\
& \leq C_{33} \cdot|A| .
\end{aligned}
$$

Proposição 6.5.1. $h:[0,1) \rightarrow[0,1)$ é $C^{1}$.

Demonstração. Pelo Lema 6.5.4, temos que $D h(x)$ existe para quase todo ponto $x \in[0,1)$. Seja $x_{0} \in[0,1)$ tal que $D h\left(x_{0}\right)$ exista. Para todo $n \geq 0$ existe $J_{n} \in \mathcal{P}^{n}$ tal que $x_{0} \in J_{n}$. Então

$$
\lim _{n \rightarrow \infty} \frac{\left|h\left(J_{n}\right)\right|}{\left|J_{n}\right|}=\operatorname{Dh}\left(x_{0}\right)
$$

Seja $\alpha \in \mathcal{A}$ tal que

$$
f^{i_{n}\left(x_{0}\right)}\left(J_{n}\right)=R^{n} f\left(I_{\alpha}^{n}\right) .
$$

Como $i_{n}\left(x_{0}\right)=i_{n}\left(h\left(x_{0}\right)\right)$, temos que $g^{i_{n}\left(x_{0}\right)}\left(h\left(J_{n}\right)\right)=R^{n} g\left(h\left(I_{\alpha}^{n}\right)\right)$. Seja $y \in J_{n}$ tal que

$$
\left|J_{n}\right|=\frac{\left|R^{n} f\left(I_{\alpha}^{n}\right)\right|}{D f^{i_{n}\left(x_{0}\right)}(y)} .
$$

Então

$$
\left|h\left(J_{n}\right)\right|=\frac{\left|R^{n} g\left(h\left(I_{\alpha}^{n}\right)\right)\right|}{D g^{i_{n}\left(x_{0}\right)}(h(y))} \cdot \exp \left(\mathrm{O}\left(\lambda_{5}^{\sqrt{n}}\right)\right) .
$$

Portanto

$$
\begin{aligned}
\frac{\left|h\left(J_{n}\right)\right|}{\left|J_{n}\right|} & =\frac{D f^{i_{n}\left(x_{0}\right)}(y)}{D g^{i_{n}\left(x_{0}\right)}(h(y))} \cdot \frac{\left|R^{n} g\left(h\left(I_{\alpha_{n}}^{n}\right)\right)\right|}{\left|R^{n} f\left(I_{\alpha}^{n}\right)\right|} \\
& =e^{\psi_{n}(y)} \cdot \frac{\left|R^{n} g\left(h\left(I_{\alpha}^{n}\right)\right)\right|}{\left|R^{n} f\left(I_{\alpha}^{n}\right)\right|} \cdot \exp \left(\mathrm{O}\left(\lambda_{5}^{\sqrt{n}}\right)\right) .
\end{aligned}
$$

Fazendo $n \rightarrow \infty$ e usando o Lema 6.5.3 obtemos

$$
\operatorname{Dh}\left(x_{0}\right)=C_{31} \cdot e^{\psi\left(x_{0}\right)} .
$$





\section{Referências Bibliográficas}

[Arn61] V. I. Arnol'd, Small denominators. I. Mapping the circle onto itself, Izv. Akad. Nauk SSSR Ser. Mat. 25 (1961), 21-86. MR 0140699 (25 \#4113)

[AV07] Artur Avila and Marcelo Viana, Simplicity of Lyapunov spectra: proof of the Zorich-Kontsevich conjecture, Acta Math. 198 (2007), no. 1, 1-56. MR 2316268 (2008m:37010)

[Bar99] Roberto Barreto, On $c^{r}$-class of conjugacy between an affine interval exchange map and its isometric model, Ph.D. thesis, IMPA, 1999.

[BHM09] X. Bressaud, P. Hubert, and A. Maass, Persistence of wandering intervals in self-similar affine interval exchange transformations, Ergodic Theory and Dynamical Systems First View (2009), 1-22.

[CG97] Ricardo Camelier and Carlos Gutierrez, Affine interval exchange transformations with wandering intervals, Ergodic Theory Dynam. Systems 17 (1997), no. 6, 1315-1338. MR MR1488320 (99e:58117)

[Cob02] Milton Cobo, Piece-wise affine maps conjugate to interval exchanges, Ergodic Theory Dynam. Systems 22 (2002), no. 2, 375-407. MR MR1898797 (2003h:37003)

[dMvS93] Welington de Melo and Sebastian van Strien, One-dimensional dynamics, Ergebnisse der Mathematik und ihrer Grenzgebiete (3) [Results in Mathematics and Related Areas (3)], vol. 25, Springer-Verlag, Berlin, 1993. MR MR1239171 (95a:58035) 
[Her79] Michael-Robert Herman, Sur la conjugaison différentiable des difféomorphismes du cercle à des rotations, Inst. Hautes Études Sci. Publ. Math. (1979), no. 49, 5-233. MR MR538680 (81h:58039)

[Her80] _ _ Résultats récents sur la conjugaison différentiable, Proceedings of the International Congress of Mathematicians (Helsinki, 1978) (Helsinki), Acad. Sci. Fennica, 1980, pp. 811-820. MR 562693 (83f:58050)

[Kea75] Michael Keane, Interval exchange transformations, Math. Z. 141 (1975), 25-31. MR MR0357739 (50 \#10207)

[Kea77] _ Non-ergodic interval exchange transformations, Israel J. Math. 26 (1977), no. 2, 188-196. MR MR0435353 (55 \#8313)

[KK03a] K. Khanin and D. Khmelev, Renormalizations and rigidity theory for circle homeomorphisms with singularities of the break type, Comm. Math. Phys. 235 (2003), no. 1, 69-124. MR 1969721 (2003m:37051)

[KK03b]_, Renormalizations and rigidity theory for circle homeomorphisms with singularities of the break type, Comm. Math. Phys. 235 (2003), no. 1, 69-124. MR MR1969721 (2003m:37051)

[KN76] Harvey B. Keynes and Dan Newton, A "minimal", non-uniquely ergodic interval exchange transformation, Math. Z. 148 (1976), no. 2, 101-105. MR MR0409766 (53 \#13518)

[KO89a] Y. Katznelson and D. Ornstein, The absolute continuity of the conjugation of certain diffeomorphisms of the circle, Ergodic Theory Dynam. Systems 9 (1989), no. 4, 681-690. MR 1036903 (91i:58122)

[KO89b] _ The differentiability of the conjugation of certain diffeomorphisms of the circle, Ergodic Theory Dynam. Systems 9 (1989), no. 4, 643-680. MR 1036902 (91i:58121)

[KR80] Michael S. Keane and Gérard Rauzy, Stricte ergodicité des échanges d'intervalles, Math. Z. 174 (1980), no. 3, 203-212. MR MR593819 (82d:28014)

[KS87a] K. M. Khanin and Ya. G. Sină̌, A new proof of M. Herman's theorem, Comm. Math. Phys. 112 (1987), no. 1, 89-101. MR 904139 (88j:58114) 
[KS87b] _ A new proof of M. Herman's theorem, Comm. Math. Phys. 112 (1987), no. 1, 89-101. MR MR904139 (88j:58114)

[KV91] K. M. Khanin and E. B. Vul, Circle homeomorphisms with weak discontinuities, Dynamical systems and statistical mechanics (Moscow, 1991), Adv. Soviet Math., vol. 3, Amer. Math. Soc., Providence, RI, 1991, Translated from the Russian by V. Nazăkinskiǔ, pp. 57-98. MR MR1118158 (92j:58089)

[Lev87] Gilbert Levitt, La décomposition dynamique et la différentiabilité des feuilletages des surfaces, Ann. Inst. Fourier (Grenoble) 37 (1987), no. 3, 85-116. MR MR916275 (88m:57035)

[LM02] Isabelle Liousse and Habib Marzougui, Échanges d'intervalles affines conjugués à des linéaires, Ergodic Theory Dynam. Systems 22 (2002), no. 2, 535-554. MR MR1898804 (2003c:37050)

[Mas82] Howard Masur, Interval exchange transformations and measured foliations, Ann. of Math. (2) 115 (1982), no. 1, 169-200. MR MR644018 (83e:28012)

[MMY05a] S. Marmi, P. Moussa, and J.-C. Yoccoz, The cohomological equation for Roth-type interval exchange maps, J. Amer. Math. Soc. 18 (2005), no. 4, 823-872 (electronic). MR MR2163864 (2006f:37006)

[MMY05b] _ Notaa do yoccoz, J. Amer. Math. Soc. 18 (2005), no. 4, 823-872 (electronic). MR MR2163864 (2006f:37006)

[MMY09] _ Affine interval exchange maps with a wandering interval, Proc. London Math. Soc. (2009), pdp037.

[MMY10] S. Marmi, P. Moussa, and J.-C. Yoccoz, Linearization of generalized interval exchange maps, ArXiv e-prints (2010).

[NR97] A. Nogueira and D. Rudolph, Topological weak-mixing of interval exchange maps, Ergodic Theory Dynam. Systems 17 (1997), no. 5, 1183-1209. MR MR1477038 (99a:58126)

[Rau79] Gérard Rauzy, Échanges d'intervalles et transformations induites, Acta Arith. 34 (1979), no. 4, 315-328. MR MR543205 (82m:10076)

[Shu87] Michael Shub, Global stability of dynamical systems, Springer-Verlag, New York, 1987, With the collaboration of Albert Fathi and Rémi Langevin, Translated from the French by Joseph Christy. MR 869255 (87m:58086) 
[Sin94a] Ya. G. Sinaŭ, Topics in ergodic theory, Princeton Mathematical Series, vol. 44, Princeton University Press, Princeton, NJ, 1994. MR MR1258087 $(95 \mathrm{j}: 28017)$

[Sin94b] _ _ Topics in ergodic theory, Princeton Mathematical Series, vol. 44, Princeton University Press, Princeton, NJ, 1994. MR MR1258087 $(95 \mathrm{j}: 28017)$

[SK89] Ya. G. Sină̌ and K. M. Khanin, Smoothness of conjugacies of diffeomorphisms of the circle with rotations, Uspekhi Mat. Nauk 44 (1989), no. 1(265), 57-82, 247. MR MR997684 (90i:58183)

[TK04] A. Yu. Teplinskiı and K. M. Khanin, Rigidity for circle diffeomorphisms with singularities, Uspekhi Mat. Nauk 59 (2004), no. 2(356), 137-160. MR 2086641 (2005b:37070)

[Vee78] William A. Veech, Interval exchange transformations, J. Analyse Math. 33 (1978), 222-272. MR MR516048 (80e:28034)

[Vee82] _ Gauss measures for transformations on the space of interval exchange maps, Ann. of Math. (2) 115 (1982), no. 1, 201-242. MR MR644019 (83g:28036b)

[Via08] Marcelo Viana, Dynamics of interval exchange transformations and teichmuller flows, 2008.

[Yoc] Jean-Christophe Yoccoz, Interval exchange maps and translation surfaces.

[Yoc84] J.-C. Yoccoz, Conjugaison différentiable des difféomorphismes du cercle dont le nombre de rotation vérifie une condition diophantienne, Ann. Sci. École Norm. Sup. (4) 17 (1984), no. 3, 333-359. MR 777374 (86j:58086)

[Zor96] Anton Zorich, Finite Gauss measure on the space of interval exchange transformations. Lyapunov exponents, Ann. Inst. Fourier (Grenoble) 46 (1996), no. 2, 325-370. MR MR1393518 (97f:58081)

[Zor97]__ Deviation for interval exchange transformations, Ergodic Theory Dynam. Systems 17 (1997), no. 6, 1477-1499. MR MR1488330 (99e:58124) 

$C_{\pi}^{s}, 59$

$C_{\pi}^{u}, 59$

$E_{0, p-1}^{c}, 62$

$E_{j, \infty}^{c}, 66$

$E_{j}^{s}, 61$

$E_{j}^{u}, 61$

$L_{n}=\left(L_{n, \alpha}\right)_{\alpha \in \mathcal{A}}, 69$

$T_{n}, 77$

$T_{n}^{\text {nor }}, 78$

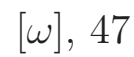

$\Omega_{\pi}, 21$

$\Pi_{d}^{g}, 20$

$\Psi_{p}(k), 62$

$\Psi_{j, \infty}, 66$

$\mathcal{T}_{n}^{m}, 79$

$\mathrm{d}_{p}, 76$

$\mathcal{B}_{k}^{r}, 22$

$\mathcal{P}^{n} ., 44$

$\operatorname{Aff}\left(\left\{\pi^{i}, \varepsilon^{i}\right\}_{i \in \mathbb{N}}, \omega\right), 73$

$\operatorname{Aff}^{(1)}\left(\left\{\pi^{i}, \varepsilon^{i}\right\}_{i \in \mathbb{N}}, \omega\right), 73$

$\partial I_{\alpha}, 15$

$\tilde{T}_{n}^{\text {nor }}, 78$

$\tilde{T}_{n}, 78$

$\tilde{\mathcal{P}}^{n}, 46$

$\mathcal{M}, 4,42$
Algoritmo de Rauzy-Veech, 13

break, 10

break point, 10

break-equivalentes, 10

classe de rotação, 20

classes de Rauzy, 16

Cociclo de Rauzy-Veech, 21

combinatória, 15

combinatória $k$-limitada, 15

Cones Invariantes, 58

conexão, 15

dado combinatorial, 12

Desigualdade de Denjoy, 26

Desigualdade de Denjoy-Koksma, 25

Direção Central, 62

distância entre duas t.i.i.'ss, 13

equação cohomológica, 83

homeomorfismos suaves por pedaços, 9

intervalo errante, 8

levantamento, 7

Métrica Projetiva, 76 
monodromia invariante, 12

número de rotação, 8

não-linearidade, 27

não-linearidade média, 27

palavra admissível, 47

permutação irredutível, 12

projectivização, 77

renormalização, 13

renormalização de Zorich, 15

Representação simbólica, 46

superfície suspensão, 19

transformação de intercâmbio de

intervalos

generalizada, 11

infinitamente renormalizável, 14

standard, 11

Suspensão de uma, 19

Transformação de Möbius, 29

transformação induzida, 13

variação, 8

variação limitada, 8

vetor inclinação, 20

vetor partição, 12

Zoom, 12 